\title{
ON CHARACTERS OF CHEVALLEY GROUPS VANISHING AT THE NON-SEMISIMPLE ELEMENTS
}

\author{
M.A. PELLEGRINI AND A.E. ZALESSKI \\ Dedicated to Lino Di Martino on the occasion of his 65th birthday
}

\begin{abstract}
Let $G$ be a finite simple group of Lie type. In this paper we study characters of $G$ that vanish at the non-semisimple elements and whose degree is equal to the order of a maximal unipotent subgroup of $G$. Such characters can be viewed as a natural generalization of the Steinberg character. For groups $G$ of small rank we also determine the characters of this degree vanishing only at the non-identity unipotent elements.
\end{abstract}

\section{INTRODUCTION}

Let $G$ be a finite simple group of Lie type in defining characteristic $p$, and let $|G|_{p}$ denote the order of a Sylow $p$-subgroup of $G$. There is a unique irreducible representation of $G$ of dimension $|G|_{p}$ over the complex numbers, and this is called the Steinberg representation. We denote here by $S t$ the character of the Steinberg representation. The Steinberg character plays a prominent role in the character theory of groups of Lie type, so this encourages one to look for generalizations of such character. In particular, in this paper we consider $p$-vanishing characters of degree $|G|_{p}$.

Let $H$ be any finite group and $p$ a prime. Elements $x \in H$ of order divisible by $p$ are called $p$-singular. Characters (in general reducible) that vanish at the $p$-singular elements of $H$ are called p-vanishing characters in this paper. The characters of projective modules over a field of characteristic $p$ are $p$-vanishing. We think that $p$ vanishing characters are of significant interest due to the connection with projective modules. In the case where $H$ is $p$-solvable, the well known result of Fong (1962) tells us that every $p$-vanishing character is the character of a projective module [5, 32.17]. In general, this is not true. To the best of our knowledge, no publications on $p$-vanishing characters appears since 1962.

As $S t$ is the character of a projective module, one may think of the characters of projective modules of dimension $|G|_{p}$ as natural analogs of the Steinberg character. Malle and Weigel [17] determined the projective modules of degree $|G|_{p}$ whose character has the trivial character $1_{G}$ as a constituent. If $G$ is a group of Lie type in characteristic $p$, the second named author showed in 24 that the restriction on

2000 Mathematics Subject Classification. 20C33, 20C40.

Key words and phrases. Chevalley groups; generalized Steinberg characters; projective modules; Gelfand-Graev characters; $p$-singular elements.

The first author was supported by FEMAT and CNPq - Brazil.

The work of the second author is a part of the collaboration project "Cohomology and representations" between the University of Milano-Bicocca (Italy) and the University of Brasilia (Brazil). 
$1_{G}$ can be dropped. In other words, the Steinberg character and the characters obtained in [17] are the only characters of projective modules of $G$ of degree $|G|_{p}$. (Note that for other primes dividing $|G|$ and for other groups the problem remains open.) In both the papers [17] and 24 the argument relies on the $p$-modular representation theory. Our results on $p$-vanishing characters of degree $|G|_{p}$ are stronger, and make no use of the theory of modular representations. Our main result is the following theorem:

Theorem 1.1. Let $G$ be a simple group of Lie type in defining characteristic $p$. If $G \in\left\{A_{n}(q), n>4,{ }^{2} A_{n}(q), n>2, B_{n}(q), q\right.$ odd $, n>5, C_{n}(q), n>2, D_{n}^{+}(q)$, $\left.n>5, D_{n}^{-}(q), n>3,{ }^{3} D_{4}(q), E_{6}(q),{ }^{2} E_{6}(q), E_{7}(q), E_{8}(q), F_{4}(q),{ }^{2} F_{4}\left(q^{2}\right), G_{2}(q)\right\}$ then the Steinberg character is the only p-vanishing character of degree $|G|_{p}$ (in particular every projective module of this dimension is the Steinberg module).

Naturally, one wishes to know whether the converse is true. Unfortunately, we have not been able to manage with the groups $B_{3}(q), B_{4}(q), B_{5}(q), D_{4}(q)$ and $D_{5}(q)$ for arbitrary $q$. For all other groups we have the full answer:

Theorem 1.2. Let $G$ be a simple group of Lie type in defining characteristic p.

(1) Groups $A_{1}(q), A_{2}(q),{ }^{2} B_{2}\left(q^{2}\right),{ }^{2} G_{2}\left(q^{2}\right)$ have reducible p-vanishing characters of degree $|G|_{p}$, where $q$ is any p-power in the former two cases, whereas in the latter two cases $q^{2}$ is an odd p-power for $p=2,3$, respectively).

(2) Groups ${ }^{2} A_{2}(q), A_{3}(q), A_{4}(q)$ have reducible p-vanishing characters of degree $|G|_{p}$ if and only if $q+1$ is divisible by 3 .

(3) Groups $C_{2}(q) \cong B_{2}(q)$ have reducible p-vanishing characters of degree $|G|_{p}$ if and only if $q+1$ is divisible by 7 .

Moreover, we give an explicit decomposition of every $p$-vanishing character as a sum of irreducible ones, and this is used in the proof of Theorem 1.1. For instance, in order to prove that $\operatorname{PSL}(6, q)$ belongs to the list of Theorem 1.1, we need to know all irreducible constituents of the $p$-vanishing characters of degree $q^{10}$ for the group $\operatorname{PSL}(5, q)$.

As long as we have obtained the decompositions of the characters in items (1), (2), (3) of Theorem [1.2 in terms of irreducible ones, we are able to compute the values of every $p$-vanishing character and compare it with those of the Steinberg character. (Full information is tabulated at the end of the paper.) We find out that every such character has real values and does not vanish at the semisimple elements. An essential feature of our results is that they are uniform with respect of $q$.

One of possible applications of our results to projective modules and the structure of the decomposition matrices can be outlined as follows. Let $\Psi, \Phi$ be the characters of projective modules; we write $\Psi>\Phi$ if $\Psi-\Phi$ is a proper character. Looking at known decomposition matrices one observes a lot of examples of projective indecomposable modules whose characters $\Psi$, $\Phi$ satisfy $\Psi>\Phi$. However, their dimensions cannot differ by $|G|_{p}$ for most groups:

Corollary 1.3. Let $G$ be a group as in Theorem 1.1. Let $\Psi>\Phi$ be the characters of two projective modules of $G$. Then either $\Psi-\Phi=$ St or $\Psi(1)-\Phi(1) \geq 2|G|_{p}$.

If $\Psi, \Phi$ are the characters of indecomposable projective modules, then $\Psi>\Phi$ means that, for every row of the decomposition matrix, the $\Phi$-entry is not greater 
than the $\Psi$-entry. The above corollary implies $\Psi(1)-\Phi(1) \geq 2|G|_{p}$ (as the first option of the corollary cannot occur).

Our proofs of Theorems 1.1 and 1.2 is based on the contemporary theory of characters of groups of Lie type. Particularly important role is played by the Gelfand-Graev characters and Harish-Chandra theory, together with regular and cuspidal characters. Note that if $\chi$ is a $p$-vanishing character, then so is the HarishChandra restriction $\bar{\chi}_{L}$ of $\chi$ to every Levi subgroup $L$ of $G$ (see [17, 24 or Lemma 2.5 below). Moreover, $\chi(1) /|G|_{p}=\bar{\chi}_{L}(1) /|L|_{p}$. Let $L_{u}$ be the subgroup of $L$ generated by the unipotent elements of $L$. Then, the restriction $\tilde{\chi}_{L}$ of $\bar{\chi}_{L}$ to $L_{u}$ is a $p$-vanishing character of $L_{u}$ of the same degree. Therefore, through induction assumption we have information on properties of $\tilde{\chi}_{L}$ for all Levi subgroups $L$ of $G$. Therefore, in order to run induction, we need first to prove Theorem 1.2 for groups of BN-pair rank 1. This case is dealt with in Section 5. Surprisingly, only for $G=S L(2, q)$ this is easy.

One could expect that these groups constitute the base of induction for our proof. However, this is not the case as the situation is more complex. Most groups of rank 2, especially, $S p(4, q),{ }^{3} D_{4}(q)$ and ${ }^{2} F_{4}\left(q^{2}\right)$ require quite a lot of computational work. Probably, performing this work manually is not realistic, but with use of CHEVIE we complete the analysis of groups of rank 2 (see Section [7).

Our results on groups of rank 2 are sufficient to run induction for unitary groups and $D_{n}^{-}(q)$. However, for the series $S L(n, q)$ we cannot run induction from $n<6$, and for the series $S p(2 n, q)$, the induction starts from $n=3$. Groups $B_{3}(q)$ and $D_{4}^{+}(q)$ should also be handled by computations. However, the CHEvIE package does not contain enough data to deal with these groups. We are still unable to complete the work for groups $B_{n}(q), n=3,4,5$, and $D_{n}^{+}(q), n=4,5$. If $n>5$, as well as for the groups $E_{6}(q), E_{7}(q), E_{8}(q)$, we do have the result stated in Theorem 1.2 due to the fact that we can use the result for $S L(n, q)$ for induction purpose.

For computer computations we use the program package CHEVIE, created by a research group at University of Aachen. This is better adapted to deal with groups with connected center such as $G L(n, q)$ or $C S p(2 n, q)$, the conformal symplectic group. In particular, we elaborate in details the cases $G L(6, q)$ and $C S p(6, q)$. We expect that every $p$-vanishing character of a simple group $G$ of Lie type in defining characteristic $p$ is the restriction to $G$ of a $p$-vanishing character of a suitable group with connected center. Unfortunately, we have not been able to prove this. Instead, we prove that every $p$-vanishing character of $G$ is the restriction to $G$ of a $S y l_{p^{-}}$ vanishing character of a group $H$ with connected center (Proposition 6.2). A $S y l_{p^{-}}$ vanishing character of a finite group $G$ means a character vanishing at all nonidentity elements of a Sylow $p$-subgroup of $G$. This forces us to pay significant attention to determining $S y l_{p}$-vanishing characters of degree $|G|_{p}$ for groups $G$ of small rank. In fact, we determine all $S y l_{p}$-vanishing characters of degree $|H|_{p}$ for quasi-simple groups $H$ of BN-pair rank 1 and 2 and the groups $H=C S p(6, q), q$ odd, $G L(n, q), n=4,5,6, U(n, q), n=3,4,5$, and use certain elements of $H$ to rule out the characters that are not $p$-vanishing. This gives us the list of the $p$ vanishing characters of degree $|H|_{p}$ for $H$. Our results on $S y l_{p}$-vanishing characters are auxiliary for the purpose of this paper, however, we expect that they will play some role in general theory. 
In order to perform the inductive step, we have to convert information on $\tilde{\chi}_{L}$ for various Levi subgroups $L$ to a certain conclusion on $\chi$. Apart from HarishChandra induction (see [6]), we use some basic theory of general Gelfand-Graev characters. We define a (general) Gelfand-Graev character to be $\nu^{G}$, where $\nu$ is a linear character of a Sylow $p$-subgroup of $G$ for $p$ being the defining characteristic of $G$. (The traditional definition of a Gelfand-Graev character (see [7, 14.29] or [2, p. 254]) requires a certain non-degeneracy condition on $\nu$, which is dropped in our definition. Gelfand-Graev characters satisfying the non-degeneracy condition are called here non-degenerate as in [14.) Then we have $\left(\chi, \nu^{G}\right)=\chi(1) /|G|_{p}$ for every $p$-vanishing character $\chi$ (Lemma 2.4). This allows us to take some control of the irreducible constituents of $\chi$ that are common with $\nu^{G}$ for some $\nu$.

For groups other than $S L(n, q)$ there are irreducible characters that do not belong to any $\nu^{G}$. We are unable to control such constituents of $\chi$, and this is a source of certain difficulties. Strictly speaking, our non-computational results only concern with the "Gelfand-Graev part" $\gamma(\chi)$ of $\chi$, where $\gamma(\chi)$ is the sum of all irreducible constituents of $\chi$ that occur in $\nu^{G}$ for some $\nu$.

The formula $\left(\chi, \nu^{G}\right)=\chi(1) /|G|_{p}$ allows us to bound the multiplicities of the constituents common for $\chi$ and $\nu^{G}$ but does not help in identifying them. In particular, if $G$ is with connected center of BN-pair rank 2, and if $\chi$ is $p$-vanishing character of degree $|G|_{p}$ then the number of the constituents of $\chi$ in question is at most 4, however, even in these cases we cannot avoid computations with explicit character tables. For projective module characters of degree $|G|_{p}$ this has been done manually, see [17] and 24]. For some groups this can be done manually in our situation too, however, some groups of rank 2 such as ${ }^{2} F_{4}\left(q^{2}\right)$ seem to require unbearable volume of manual work.

The induction step would be easy if some Levi subgroup of $G$ had no $p$-vanishing character of degree $|G|_{p}$. However, this never happens, as the defect zero irreducible characters of $L$ (in particular, the Steinberg one) are $p$-vanishing of degree $|L|_{p}$. In some cases of interest for every maximal Levi subgroup $L$ every $p$-vanishing character of degree $|G|_{p}$ is of defect 0 . This leads to the natural question: is a $p$-vanishing character irreducible if the Harish-Chandra restriction of it to every Levi subgroup is a sum of defect 0 irreducible characters? In the case of projective indecomposable modules the Brauer-Nesbitt correspondence reduces this to a similar question for irreducible modules, which are easy to handle (see [24). In our case no analog of the Brauer-Nesbitt correspondence is available. This explains why one cannot mimic any machinery from the theory of projective modules to deal with $p$-vanishing characters.

Now we continue our discussion of groups of small rank. As mentioned above, the task is to determine all $S y l_{p}$-vanishing characters of degree $|G|_{p}$. Technical difficulties are forced us to look for tools for simplifying computational work. We have found an advantage to determine first the characters $\chi$ of $G$ such that $\left.\chi\right|_{U}$ is the character of the regular representation of $U$, where $U$ is a Sylow $p$-subgroup of $G$. For groups of small rank the number of such characters is not large, and we are able to check which of them are $p$-vanishing. The matter is that the CHEVIE format of the character table is better adapted to deal with $S y l_{p}$-vanishing characters, as CHEVIE organizes them in sets such that all characters in the same set have the same restriction to $U$. In this way we found another phenomenon of general interest. Specifically, we discover a considerable amount of formulas of type $\left.\chi_{1}\right|_{U}=$ 
$\left.\chi_{2}\right|_{U}+\left.\chi_{3}\right|_{U}$, where $\chi_{i}(i=1,2,3)$ are irreducible characters of $G$. Therefore, if $\chi_{1}$ occurs in a decomposition of some $S y l_{p}$-vanishing character in terms of irreducible characters, then the substitution $\chi_{2}+\chi_{3}$ for $\chi_{1}$ yields another decomposition, in which the degrees of $\chi_{2}, \chi_{3}$ are less than that of $\chi_{1}$. We did not try in this paper to obtain all formulas of this kind, but those we have got are collected in the tables at the end of the paper. Partially, we expose them as tools helping the reader to control our computations. Another purpose is to provide experimental material for theoretical analysis.

Note that some formulas provided in the tables involve more than three terms (see for instance Table11.3. A for $G={ }^{2} B_{2}\left(q^{2}\right)$ ).

Notation. $\mathbb{C}$ is the complex number field, $\mathbb{Z}$ is the ring of integers and $F_{q}$ is the finite field of $q$ elements. For a positive integer $m$ we define $\mathbb{Z}_{m}=\mathbb{Z} / m \mathbb{Z}$. We often view $\mathbb{Z}_{m}$ as the set of integers $\{1, \ldots, m\}$.

For a finite group $G$ we denote by $G^{\prime}, Z(G)$ and $|G|$ the derived subgroup, the center and the order of $G$, respectively. If $p$ is a prime then $|G|_{p}$ is the $p$-part of $|G|$ and also the order of every Sylow $p$-subgroup of $G$. A $p^{\prime}$-group means a finite group with no element of order $p$. If $g \in G$ then $|g|$ is the order of $g$. All representations and modules are over $\mathbb{C}$ (unless otherwise stated). Therefore, we take liberty to use the term ' $G$-module'. All modules are assumed to be finitely generated. The character of the regular representation of $G$ is denoted by $\rho_{G}^{\text {reg }}$ and the trivial onedimensional representation is denoted by $1_{G}$. We also use $1_{G}$ to denote the trivial one-dimensional module and its character. The inner product of class functions $\eta, \eta^{\prime}$ is denoted by $\left(\eta, \eta^{\prime}\right)$.

The set of irreducible characters of $G$ is denoted by $\operatorname{Irr} G$, and any integral linear combination of elements of $\operatorname{Irr} G$ is called a generalized character of $G$.

Let $H$ be a subgroup of $G$ and $N$ a normal subgroup of $H$. Let $M$ be an $H$ module over a field. Then $C_{M}(N)$ is an $H$-module, and when it is viewed as $H / N$ module, it is denoted by $\bar{M}_{H / N}$ (or $\bar{M}$ ), and called the Harish-Chandra restriction of $M$ to $H / N$. Conversely, given an $H / N$-module $D$, one can view it as an $H$-module with trivial action of $N$. Then the induced $G$-module $D^{G}$ (when $D$ is viewed as an $H$-module) is denoted by $D^{\# G}$ and called Harish-Chandra induced from $D$. For details see $[6$, p. $667, \S 70 \mathrm{~A}]$, where these operations are called generalized restriction and induction. They correspond to similar operations on characters (or Brauer characters). The Harish-Chandra restriction and induction extend by linearity to class functions on $G$ with values in $\mathbb{C}$. So if $\chi$ is a class function on $G$ then $\bar{\chi}_{H / N}$ is the corresponding Harish-Chandra restriction of $\chi$ to $H / N$, and if $\lambda$ is a class function on $H / N$ then $\lambda^{\# G}$ denotes the Harish-Chandra induced class function on $G$. Let $\eta$ be a class function on $G$. The formula $\left(\lambda^{\# G}, \eta\right)=\left(\lambda, \bar{\chi}_{H / N}\right)$ is an easy consequence of the Frobenius reciprocity and called the Harish-Chandra reciprocity. (This is the formula 70.1(iii) in [6, p. 668].) Given a class function $f$ on $G$ define a function on $H$ by $h \rightarrow \frac{1}{|N|} \sum_{n \in N} f(h n)(h \in H)$. This function is constant on the cosets $h N$ and therefore defines a function on $H / N$ called the truncation of $f$ to $H / N$ with respect to $N$ (see [6, 70.4] or [2, pp. $262-283$ ]). Sometimes we also call the truncation the function $h \rightarrow \frac{1}{|N|} \sum_{n \in N} f(h n)$ on $H$ (which hopefully does not lead to a confusion). Note that the truncation and the Harish-Chandra restriction of a character coincide.

Let $\mathbf{H}$ be a reductive algebraic group in defining characteristic $p>0$. An algebraic group endomorphism $\mathrm{Fr}: \mathbf{H} \rightarrow \mathbf{H}$ is called Frobenius if the the subgroup 
$\mathbf{H}^{F r}=\{h \in \mathbf{H}: \operatorname{Fr}(h)=h\}$ is finite. This subgroup is called a finite reductive group, and $p$ is referred as the defining characteristic of $\mathbf{H}^{F r}$ too. Every finite reductive group is a group with BN-pair, and our use of terms 'Borel, parabolic and Levi subgroup, Weyl group' is as in the theory of groups with BN-pair [6, §65]. More detailed notation will be introduced later.

If $\mathbf{H}$ is a simple algebraic group of universal type then we refer to $\mathbf{H}^{F r}$ as a Chevalley group. (Some authors additionally assume that $\mathrm{Fr}$ acts trivially on the Weyl group of $\mathbf{H}$, but we prefer to refer to such groups as non-twisted Chevalley groups.)

Let $H=\mathbf{H}^{F r}$ be a finite reductive group. We use some results on the Curtis duality (called also the Curtis-Alvis duality) for the class functions on $H$, see 6 , $\S 71 \mathrm{~A}]$. If $\phi$ is a class function on $H$, we denote by $D(\phi)$ the Curtis dual of $\phi$. If $D(\phi)= \pm \phi$ then we call $\phi$ Curtis self-dual.

Our notation for simple groups of Lie type are as in 2, however, for classical groups we also use the traditional notation such as $\operatorname{PSU}(n, q)$ for the unitary group. Similarly, for quasi-simple groups and classical groups with connected center. For instance, $C S p(2 n, q)$ denotes the conformal symplectic group.

\section{2. p-VANISHING CHARACTERS AND THE HARISH-ChANDRA RESTRICTION}

We start with few observations valid for every finite group.

2.1. $p$-vanishing and $S y l_{p}$-vanishing characters. Let $\phi$ be a class function on a finite group $G$, and let $p$ a prime dividing $|G|$. We say that $\phi$ is $p$-vanishing if $\phi(g)=0$ whenever $p$ divides $|g|(g \in G)$. This also applies to characters and to generalized characters. For instance, the character of the regular module is $p$ vanishing for every prime $p$. The characters of projective $G$-modules over a field of characteristic $p$ are $p$-vanishing. It is well known that every $p$-vanishing function is a linear combination of the characters of projective indecomposable modules, and every $p$-vanishing generalized character is an integer linear combination of them, see [8, Ch. IV, Corollary 3.13] or [5, Theorem 18.26]. Furthermore, we say that $\phi$ is $S_{y l}$-vanishing if $\phi(g)=0$ whenever $g \neq 1$ belongs to a Sylow $p$-subgroup of $G$. Obviously, $p$-vanishing class functions are $S y l_{p}$-vanishing, but the converse is not always true. However, for irreducible characters, the converse is true (well known). (If $G=P S L(3, q), q=p^{k}$, then there are $S y l_{p}$-vanishing reducible characters of degree $|G|_{p}$ that are not $p$-vanishing.)

If $\chi$ is a $S y l_{p}$-vanishing proper or generalized character of $G$ then $\chi(1)$ is a multiple of $|G|_{p}$, and we set $c_{\chi}=\chi(1) /|G|_{p}$. This notation allows us to express certain information in a more compact form.

A proper character $\chi$ is $S y l_{p}$-vanishing if and only if the restriction of $\chi$ to a Sylow $p$-subgroup $U$ of $G$ is the character of a free $U$-module.

A proper $p$-vanishing (resp. $S y l_{p}$-vanishing) character $\chi$ is called minimal if whenever $\chi=\chi_{1}+\chi_{2}$, where $\chi_{1}, \chi_{2}$ are proper characters of $G$, then neither $\chi_{1}$ nor $\chi_{2}$ is $p$-vanishing (respectively, $S y l_{p}$-vanishing).

Obviously, irreducible $p$-vanishing and $S y l_{p}$-vanishing characters are minimal and have defect 0 . The converse is not true, for instance, every group $S L(2, q)$, $q=p^{k}$, has a reducible $p$-vanishing character of degree $q$. Thus, minimal $p$-vanishing characters are analogous to irreducible characters of defect 0 . Other examples of minimal $p$-vanishing and $S y l_{p}$-vanishing characters $\chi$ are those with $c_{\chi}=1$. Note that $c_{\chi}=1$ means that $\left.\chi\right|_{U}=\rho_{U}^{r e g}$, where $U$ is a Sylow $p$-subgroup of $G$. 
Lemma 2.1. Let $G$ be a finite group and let $\chi$ be a minimal p-vanishing character of $G$. Then all irreducible constituents of $\chi$ are in the same block.

Proof. This follows from [8, Ch.IV, Lemma 3.14].

Lemma 2.1 allows one to assign a $p$-block to every minimal $p$-vanishing character. Note that the lemma is not true for $S y l_{p}$-vanishing characters, even for $S L(2, q)$.

Corollary 2.2. Let $G$ be a perfect finite group, and let $\chi$ be a minimal p-vanishing character of $G$. Let $z \in Z(G)$. Suppose that $(\chi(1),|z|)=1$. Then $z$ belongs to the kernel of $\chi$.

Proof. Let $\phi$ be a representation of $G$ with character $\chi$. It follows from Lemma 2.1 that $\phi(z)=\zeta(z) \cdot \operatorname{Id}$ for some $\zeta \in \operatorname{Irr} Z(G)$. Then $1=\operatorname{det} \phi(z)=\zeta(z)^{n}$, where $n=\chi(1)$. This implies $\zeta(z)=1$.

Lemma 2.3. Let $G$ be a finite group with normal subgroup $N$, and let $M$ be a free $G$-module of rank $r$. Then $C_{M}(N)$ is a free $G / N$-module of rank $r$.

Consequently, if $\chi$ is a Syl $l_{p}$-vanishing character then $\bar{\chi}_{G / N}$ is Syl $p_{p}$-vanishing and $c_{\chi}=c_{\bar{\chi}_{G / N}}$.

Proof. The first claim is obvious for $r=1$, and easily implies the statement. To get the second claim, apply the first one to a Sylow $p$-subgroup $U$ of $G$ in place of $G$ and to $N \cap U$ in place of $N$.

Lemma 2.4. Let $G$ be a finite group and $U$ a Sylow p-subgroup of $G$. Let $\eta$ be a Syl $l_{p}$-vanishing class function on $G$. Let $\tau$ be an irreducible character of $U$. Then $\left(\eta, \tau^{G}\right)=\eta(1) \tau(1) /|U|$, and hence $c_{\eta} \cdot \tau(1)=\left(\eta, \tau^{G}\right)$.

Proof. We have $\left(\eta, \tau^{G}\right)=\left(\left.\eta\right|_{U}, \tau\right)=\eta(1) \tau(1) /|U|$.

2.2. The Harish-Chandra restriction. Our standard assumption here is that $G$ is a finite group, $U$ a Sylow $p$-subgroup of $G, P \subset G$ is a subgroup containing $U$ and $L=P / O_{p}(P)$. (Later on, we specify $G$ to be a finite Chevalley group, $P$ a parabolic subgroup of $G$ and $L$ a Levi subgroup of $P$.) Recall that for a character (or a class function) $\chi$ of $G$ we denote by $\bar{\chi}_{L}$ the truncation of $\chi$ to $L$.

The following easy lemma is used in many situations.

Lemma 2.5. Let $G, P, L$ be as above.

(1) Let $\chi$ be a p-vanishing (resp., Syl $l_{p}$-vanishing) character of $G$. Then $\bar{\chi}_{L}$ is a p-vanishing (resp., Syl $l_{p}$-vanishing) character of $L$ and $c_{\chi}=c_{\bar{\chi}_{L}}$. (In particular, $\bar{\chi}_{L} \neq 0$.)

(2) Let $\eta$ be an irreducible constituent of $\bar{\chi}_{L}$. Then $\left(\bar{\chi}_{L}, \eta\right)=\left(\chi, \eta^{\# G}\right)>0$.

Proof. (1) The statement on $S y l_{p}$-vanishing characters and the equality follows from Lemma 2.3 applied to $P$. Assume that $\chi$ is $p$-vanishing. Let $M$ be a $\mathbb{C} P$-module with character $\chi$, and let $R$ be the fixed point subspace of $U$ on $M$. Note that $r \in R$ if and only if $r=\frac{1}{|U|} \sum_{u \in U} u m$ for some $m \in M$. Let $g \in P, u \in U$. Suppose that the projection of $g$, and hence of $g u$, into $L$ is not a $p^{\prime}$-element. (Note that the projections of $g u$ and $g$ into $L$ coincide.) It follows that $\bar{\chi}_{L}(g)=\frac{1}{|U|} \sum_{u \in U} \chi(g u)=$ 0 by assumption, whence the first claim.

(2) follows from Harish-Chandra reciprocity. 
Lemma 2.6. Let $G$ be a finite group and $U$ a Sylow p-subgroup of $G$. Let $\chi$ be a Syl $l_{p}$-vanishing character of $G$. The following are equivalent:

(a) $\chi(1)=|G|_{p}=|U|$;

(b) $\bar{\chi}_{N_{G}(U) / U}(1)=1$. In other words, the truncation of $\chi$ over $U$ is a linear character of $N_{G}(U) / U$.

\section{Gelfand-Graev characters}

In this section $H$ is a finite reductive group in defining characteristic $p$, and $U$ a Sylow $p$-subgroup of $H$. Let $\lambda$ be a character of $U$ with $\lambda(1)=1$. Then we call $\lambda^{H}$ a (general) Gelfand-Graev character of $H$. The original Gelfand-Graev characters are called non-degenerate in 14, and we use this term to specify the original Gelfand-Graev characters within the set of (general) Gelfand-Graev characters. (If $H$ is not with connected center, there can be several non-degenerate Gelfand-Graev characters [7.) Gelfand-Graev characters play a significant role in this paper. The cause of this is partially explained by the following lemma which is in fact is a special case of Lemma 2.4 .

Lemma 3.1. Let $\chi$ be a Syl $l_{p}$-vanishing character of $H$ of degree $|H|_{p}$. Then any Gelfand-Graev character has exactly one common irreducible constituent with $\chi$, and this occurs with multiplicity 1 in each of them. In particular, St is a constituent of every Gelfand-Graev character.

Let $\chi$ be a $S y l_{p}$-vanishing character. Then we can write $\chi=\gamma(\chi)+\gamma^{\prime}(\chi)$, where $\gamma(\chi)$ is the sum of all irreducible constituents of $\chi$ that occur in some GelfandGraev character, and $\gamma^{\prime}(\chi)$ is the sum of the remaining terms. Clearly, $\gamma(\chi)$ is the sum of the irreducible constituents $\tau$ of $\chi$ such that $\left.\tau\right|_{U}$ contains a linear character of $U$.

Corollary 3.2. The number of irreducible constituents of $\gamma(\chi)$ does not exceed the number of distinct Gelfand-Graev characters.

Lemma 3.3. Let $\chi$ be a Syl $l_{p}$-vanishing character of $H$ of degree $|H|_{p}$. Then $\gamma(\chi)$ is multiplicity free.

Proof. This follows from the equality $\left.\chi\right|_{U}=\rho_{U}^{r e g}$, and is also an immediate consequence of Lemma 3.1 .

Remark 3.4. The character $\gamma^{\prime}$ is not always multiplicity free. For instance, for $H=P S U(3,5)$, in notation of Atlas [3], the character $\chi_{1}+2 \chi_{2}+\chi_{4}+\chi_{5}+\chi_{6}$ is 5 -vanishing of degree $|H|_{5}=125$.

The Gelfand-Graev characters can be characterized as follows:

Lemma 3.5 ([14, Lemma 2.3]). Suppose that $H$ is with connected center, with no component of type $B_{n}(2), C_{n}(2), F_{4}(2), G_{2}(2), G_{2}(3),{ }^{2} B_{2}(2),{ }^{2} F_{4}(2),{ }^{2} G_{2}(3)$. Let $\lambda$ be a linear character of $U$. Then $\lambda^{H}=\Gamma_{L}^{\# H}$, where $L$ is some Levi subgroup of $H$ and $\Gamma_{L}$ is the non-degenerate Gelfand-Graev character of $L$. Conversely, if $\Gamma_{L}$ is the non-degenerate Gelfand-Graev character of $L$, then $\Gamma_{L}^{\# H}=\lambda^{H}$ for some linear character $\lambda$ of $U$.

Remark 3.6. The exclusions in the above lemma correspond to the cases where $U \neq U_{1}$ in notation of [14, p. 350]. The statement in [14 does not exclude the groups $H$ with components of type ${ }^{2} B_{2}(2),{ }^{2} F_{4}(2),{ }^{2} G_{2}(3)$, however, we think that this is required. 
Let $\mu \in \operatorname{Irr} H$ and let $L$ be a Levi subgroup of $H$. Suppose that $\mu$ is not a constituent of any Gelfand-Graev character of $H$. Then all irreducible constituents of $\bar{\mu}_{L}$ belong to $\gamma^{\prime}\left(\bar{\chi}_{L}\right)$. Indeed, suppose the contrary, and let $\lambda$ be an irreducible constituent of $\bar{\mu}_{L}$ belonging to $\gamma\left(\bar{\chi}_{L}\right)$. This means that $\lambda$ is a constituent of $\Gamma_{K}^{\# L}$ for some Levi subgroup $K$ of $L$ (and hence of $H$ ) and a non-degenerate GelfandGraev character $\Gamma_{K}$ of $K$. Note that $\left(\mu, \lambda^{\# H}\right)=\left(\bar{\mu}_{L}, \lambda\right)>0$ by Harish-Chandra reciprocity. Therefore, $\mu$ is a constituent of $\Gamma_{K}^{\# H}$ by transitivity of Harish-Chandra induction. Hence, by Lemma 3.5, we get a contradiction.

Note that a regular character $\sigma$ is cuspidal if and only if $\sigma$ is semisimple. Indeed, consider the Curtis dual $D(\sigma)$ of $\sigma$. As $\sigma$ is regular, $\varepsilon D(\sigma)$ is semisimple for some $\varepsilon= \pm 1$ [7, 14.39]. However, $\sigma$ is cuspidal if and only if $D(\sigma)= \pm \sigma$ [6, p. 690], so the claim follows.

Lemma 3.7. Let $H$ be as in Lemma 3.5. Then the number of distinct GelfandGraev characters equals the number of non-conjugate Levi subgroups of $H$.

Proof. By Lemma 3.5, every Gelfand-Graev character is of shape $\Gamma_{L}^{\# H}$, where $\Gamma_{L}$ is the non-degenerate Gelfand-Graev character of $L$. (Here $L$ can coincide with $H$.) Since $H$ is with connected center then so is $L$ (see [2, 8.1.4]), and hence $L$ has a unique non-degenerate Gelfand-Graev character. Clearly, if Levi subgroups $L, M$ of $H$ are conjugate then $\Gamma_{L}^{\# H}$ coincides with $\Gamma_{M}^{\# H}$. (Note that $\Gamma_{L}^{\# H}$ does not depend on the choice of the parabolic subgroup which defines $L$ as a Levi subgroup [6, 70.10].) Suppose that $L, M$ are not conjugate. It is known that each $L, M$ has a cuspidal regular character $\eta_{L}, \eta_{M}$, say. (Indeed, if a maximal torus $T^{*}$ of $H^{*}$ contains an element $s$ in general position then the regular character $\rho_{s}$ corresponding to $s$ is cuspidal if and only if $T^{*}$ contained in no proper Levi subgroup of $H^{*}$ (see [2, 9.3.2]). It is shown by Veldkamp [23, p. 391] that a so called Coxeter torus $T^{*}$ is not contained in any proper Levi subgroup of $H^{*}$ and contains an element in general position; it is also known that an element in general position is contained in a unique maximal torus [23, p. 390].) Then $\eta_{L}^{\# H}, \eta_{M}^{\# H}$ are disjoint, and their irreducible constituents are contained in $\Gamma_{L}^{\# H}, \Gamma_{M}^{\# H}$, respectively. So $\Gamma_{L}^{\# H} \neq \Gamma_{M}^{\# H}$, and the result follows.

Remark 3.8. One observes that this result is not valid for $H={ }^{2} B_{2}(2),{ }^{2} G_{2}(3),{ }^{2} F_{4}(2)$.

The following result is contained in [14, Propositions 2.4, 2.5, Corollary 2.6].

Lemma 3.9. Let $H$ be as in Lemma 3.5, and $\tau \in \operatorname{Irr} H$. Then the following are equivalent:

(1) $\tau$ is a constituent of some Gelfand-Graev character of $H$;

(2) $\tau$ is a constituent of $\lambda^{\# H}$, where $\lambda$ is a cuspidal regular character of a Levi subgroup of some parabolic subgroup of $H$.

Observe that the character $\lambda$ in Lemma 3.9 is both regular and semisimple because every cuspidal regular character is semisimple (see comments prior Lemma 3.7).

Distinct Gelfand-Graev characters have common irreducible constituents. Recall that a Harish-Chandra series of $H$ is the set of irreducible constituents of a Harish-Chandra induced character $\lambda^{\# H}$, where $L$ is a Levi subgroup of a parabolic subgroup $P$ of $H$ (possibly $P=H$ ) and $\lambda$ is a cuspidal character of $L$. Two Harish-Chandra series either coincide or are disjoint, see [6, 70.15A]. Therefore, if 
$\lambda$ is cuspidal then the irreducible constituents of $\lambda^{\# H}$ do not occur in the other Gelfand-Graev characters.

In addition, if $\sigma$ is a regular cuspidal character of $L$ then the irreducible constituents of $\sigma^{\# H}$ are in bijection with $\operatorname{Irr} W_{\sigma}$, where $W_{\sigma}$ is a certain subgroup of $W$ generated by reflections. More precisely, $W$ acts on the roots of the corresponding algebraic group, and $L$ is defined by a subset $J$ of simple roots. Then $W=\{w \in W: w(J)=J$ and $w(\sigma)=\sigma\}$.

Proposition 3.10. Assume $H$ to be with connected center and has no component of type $B_{n}(2), C_{n}(2),{ }^{2} B_{2}(2), F_{4}(2),{ }^{2} F_{4}(2), G_{2}(2), G_{2}(3)$ and ${ }^{2} G_{2}(3)$. Let $\chi$ be a Syl $_{p}$-vanishing character of $H$ of degree $|H|_{p}$.

(1) For every irreducible constituent of $\tau$ of $\gamma(\chi)$ there are a unique Levi subgroup $L$ of $H$ and a unique cuspidal regular character $\rho$ of $L$ such that $\left(\tau, \rho^{\# H}\right)>0$. Moreover, $\left(\tau, \rho^{\# H}\right)=1$.

(2) Let $l(\chi)$ be the number of non-conjugate Levi subgroups of $H$ such that $\bar{\chi}_{L}$ contains a cuspidal regular character of $L$. Then the number of irreducible constituents of $\gamma(\chi)$ equals $l(\chi)$.

Proof. Let $\left\{\nu_{1}, \ldots, \nu_{k}\right\}$ be a maximal set of linear characters of $U$ such that $\nu_{i}^{H} \neq$ $\nu_{j}^{H}$ for $i \neq j$. By Lemma 3.5, $\nu_{i}^{H}=\Gamma_{L}^{\# H}$ for some Levi subgroup $L$, and by Lemma 3.7. the set $\left\{\nu_{1}, \ldots, \nu_{k}\right\}$ is in bijection with the set of non-conjugate Levi subgroups. Let $L_{i}$ correspond to $\nu_{i}$ for $i=1, \ldots, k$. As $\left(\chi, \nu_{i}^{H}\right)=1$ (Lemma 3.1), we may denote by $\chi_{i}$ the constituent of $\chi$ common with $\nu_{i}^{H}$. (So $\chi_{i}$ is a constituent of $\gamma(\chi)$.) The mapping $i \rightarrow \chi_{i}$ is surjective, but not injective in general.

To see when $\chi_{i}=\chi_{j}$, fix some $j$. Then $\chi_{j}$ is a constituent of $\Gamma_{L_{j}}^{\# H}$, so there is a regular character $\rho_{j}$ of $L_{j}$ such that $\left(\chi, \rho_{j}^{\# H}\right)=1$. Moreover, $\rho_{j}$ is unique. Indeed,

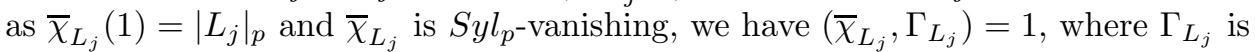
a non-degenerate Gelfand-Graev character of $L_{j}$. As $L_{j}$ is with connected center too [2, 8.1.4], $\Gamma_{L_{j}}$ is unique, and hence $\rho_{j}$ is unique.

Suppose that $\rho_{j}$ is not cuspidal. Then $\rho_{j}$ is a constituent of $\lambda_{i}^{\# L_{j}}$, where $L_{i}$ is some Levi contained in $L_{j}$ (up to conjugation) and $\lambda_{i}$ is a cuspidal regular character of $L_{i}$ (Lemma 3.9). So $\chi_{j}$ is a constituent of $\nu_{i}^{H}$ too, and hence we have $\chi_{i}=\chi_{j}$ $\left(\right.$ as $\left.\left(\chi, \nu_{i}^{H}\right)=1\right)$. Thus we need not count $\nu_{j}$ such that $\bar{\chi}_{L_{j}}$ contains no cuspidal regular character of $L_{j}$, and the claim follows.

Lemma 3.11. Let $H$ be as in Proposition 3.10 and $\chi$ a Syl $l_{p}$-vanishing character of $H$. Suppose that $\gamma^{\prime}\left(\bar{\chi}_{L}\right)=0$ for every proper Levi subgroup $L$ of $H$. Then every irreducible constituent of $\gamma^{\prime}(\chi)$ is a cuspidal character.

Proof. Suppose the contrary. Let $\sigma$ be an irreducible constituent of $\gamma^{\prime}(\chi)$, which is not cuspidal. Then there exists a Levi subgroup $L$ and a cuspidal character $\mu \in \operatorname{Irr} L$ such that $\left(\sigma, \mu^{\# H}\right)>0$. Therefore, $\left(\bar{\sigma}_{L}, \mu\right)=\left(\sigma, \mu^{\# H}\right)>0$. So $\mu$ is an irreducible constituent of $\bar{\sigma}_{L}$, and hence of $\bar{\chi}_{L}$. By assumption, $\gamma^{\prime}\left(\bar{\chi}_{L}\right)=0$. This means that $\mu$ is a constituent of a Gelfand-Graev character of $L$. By Lemma 3.9, $\mu$ is a regular character of $L$ (as $\mu$ is cuspidal). Therefore, $\mu$ is a constituent of $\Gamma_{L}$, the non-degenerate Gelfand-Graev character of $L$. By Lemma 3.7, $\Gamma_{L}^{\# H}$ is a Gelfand-Graev character of $H$. As $\sigma$ is a constituent of $\mu^{\# H}$, it is also a constituent of $\Gamma_{L}^{\# H}$, that is, $\sigma$ is a summand of $\gamma(\chi)$, which is a contradiction. 
Lemma 3.12. Let $H$ be a finite reductive group and let $\tau, \sigma$ be distinct irreducible constituents of $\gamma(\chi)$ for a Syl ${ }_{p}$-vanishing character $\chi$ of degree $|H|_{p}$. Then $\left.\tau\right|_{U}$ and $\left.\sigma\right|_{U}$ have no common constituent of degree 1 , in particular, $\left.\tau\right|_{U} \neq\left.\sigma\right|_{U}$.

Proof. Indeed, suppose the contrary. Then $\left(\left.\tau\right|_{U}, \nu\right)>0$ and $\left(\left.\sigma\right|_{U}, \nu\right)>0$ for some linear character $\nu$ of $U$. As $\left.\chi\right|_{U}=\rho_{U}^{r e g}$, we have $\left(\left.\chi\right|_{U}, \nu\right)=1$, and the claim follows.

Lemma 3.13. Let $H=G L(n, q)$ or $S L(n, q)$. Then every irreducible character of $H$ is a constituent of a Gelfand-Graev character.

Proof. For $H=G L(n, q)$ the result is stated in the original paper by Gelfand and Graev [10, and this implies the one for $G=H^{\prime}=S L(n, q)$. Indeed, let $U$ be a Sylow $p$-subgroup of $G$. Let $\lambda \in \operatorname{Irr} U$ with $\lambda(1)=1$. By Mackey's lemma, $\left.\left(\lambda^{H}\right)\right|_{G}=\sum_{g}\left(\lambda^{g}\right)^{G}$, where $g$ are the representatives of the double cosets of $G$ and $U$ in $H$. Since $H=G \cdot T$, where $T$ is a maximal torus of the Borel subgroup of $H$ containing $U$ [16, Lemma 24.12], we may choose $g$ in $T$. Then $U$ is $g$-stable, $\lambda^{g}$ is a linear character of $U$ and $\left(\lambda^{g}\right)^{G}$ is a Gelfand-Graev character of $G$. So the result follows from that for $G L(n, q)$, as every irreducible character of $G$ is a constituent of the restriction to $G$ of an irreducible character of $H$.

Corollary 3.14. Let $H=G L_{n}(q)$ or $S L_{n}(q)$, and let $\chi$ be a Syl $l_{p}$-vanishing character of $H$ of degree $|H|_{p}$. Then $\chi=\gamma(\chi)$, and hence $\chi$ is multiplicity free.

Proof. This follows from Lemmas 3.13 and 3.3 .

\section{4. $S y l_{p}$-DECOMPOSABLE IRREDUCIBLE CHARACTERS}

4.1. General observations. Let $\tau$ be an irreducible character of a finite group $G$. Let $U$ be a Sylow $p$-subgroup of $G$. We say that $\tau$ is $S y l_{p}$-decomposable if there are irreducible characters $\chi_{1}, \ldots, \chi_{k}$ of $G$ (not necessarily distinct) such that $\tau(u)=\chi_{1}(u)+\cdots+\chi_{k}(u)$ for every element $u \in U$. (Equivalently, $\tau-\chi_{1}-\cdots-\chi_{k}$ vanishes on $U$.) We express this by writing $\tau \equiv \chi_{1}+\cdots+\chi_{k}(\bmod U)$, and call this expression a $S y l_{p}$-decomposition of $\tau$. Note that some characters of $G$ can be Syl $l_{p}$-equivalent, that is, they take the same value at every $u \in U$.

The problem of determining the $S y l_{p}$-vanishing characters of degree $|U|$ discussed above for finite reductive groups in defining characteristic $p$ can be restated as that of finding all $S y l_{p}$-decompositions of the Steinberg character of $G$. We find out that the knowledge of $S y l_{p}$-decompositions for characters $\tau$ of degree less that $|U|$ is very helpful for determining the $S y l_{p}$-vanishing characters, seems to be unavoidable for most groups of rank greater than 1. Morever, we feel that they are of certain independent interest. Because of these we perform an extensive computations of them, and collect the relevant results in the tables at the end of the paper.

We collect here some general facts on $S y l_{p}$-decompositions for finite reductive groups $H$.

Recall that, if $\phi$ is a class function on $H$ then $D(\phi)$ denotes the Curtis dual of $\phi$. If $D(\phi)= \pm \phi$ then we call $\phi$ Curtis self-dual. The following well known fact can be easily deduced from the definitions of the duality operation [6, 71.2] and the Steinberg character [6, 71.18].

Lemma 4.1. Let $\phi$ be a class function on $H$ constant on $U$. Then $D(\phi)(g)=$ $S t(g) \phi(g)$ for all $g \in H$. In particular, if $\phi(u)=0$ for all $u \in U$ then $D(\phi)(u)=0$ for all $u \in U$. 
Lemma 4.2. Suppose $H$ is with connected center. Let $\chi_{1}, \chi_{2}, \chi_{3} \in \operatorname{Irr} H$. Suppose that $\chi_{1} \equiv \chi_{2}+\chi_{3}(\bmod U)$ is a Syl $l_{p}$-decomposition of $\chi_{1}$.

(1) At least one of the characters $\chi_{i}, 1 \leq i \leq 3$, is not regular.

(2) At least one of the characters $\chi_{i}, 1 \leq i \leq 3$, is not semisimple.

(3) If every $\chi_{i}$ is either regular or semisimple then at least one of them is regular and semisimple (and hence Curtis self-dual).

(4) $D\left(\chi_{1}\right)-D\left(\chi_{2}\right)-D\left(\chi_{3}\right)$ is a Syl $l_{p}$-vanishing generalized character of degree 0 . Let $\chi_{i}^{\prime}= \pm D\left(\chi_{i}\right)$ be a proper character, $i=1,2,3$. Then reordering of the $\chi_{i}^{\prime}$ yields a Syl $p_{p}$-decomposition (which may coincide with that in the assumption).

Proof. Note that $\chi_{1}-\chi_{2}-\chi_{3}$ is a $S y l_{p}$-vanishing generalized character of degree 0 . By Lemma 4.1, so is $D\left(\chi_{1}-\chi_{2}-\chi_{3}\right)$, which implies (4). Let $\Gamma$ be the non-degenerate Gelfand-Graev character of $H$.

(1) If all $\chi_{i}$ are regular, then, taking the inner product of $\chi_{1}-\chi_{2}-\chi_{3}$ with $\Gamma$, we get $1-1-1=0$, which is absurd.

(2) Suppose all of them are semisimple. As $\pm D\left(\chi_{i}\right)$ is a regular character for $i=1,2,3$, the inner product with $\Gamma$ yields again $\pm 1 \pm 1 \pm 1=0$, a contradiction.

(3) Suppose that none of the $\chi_{i}$ 's is regular and semisimple simultaneously. If one of the characters is regular and the other two are not regular, the inner product with $\Gamma$ yields a contradiction. So there are two regular characters among them, and they are not semisimple. Then $D\left(\chi_{1}\right)-D\left(\chi_{2}\right)-D\left(\chi_{3}\right)$ is $S y l_{p}$-vanishing, and two characters among $D\left(\chi_{1}\right),-D\left(\chi_{2}\right),-D\left(\chi_{3}\right)$ are semisimple and not regular, and one is regular. Again, the inner product with $\Gamma$ yields a contradiction.

Lemma 4.3. Suppose $H$ is with connected center. Let $\chi$ be a Syl $l_{p}$-vanishing character of $H$ of degree $|H|_{p}$, and let $\sigma$ be a regular character of $H$ that occurs as a constituent of $\gamma(\chi)$. Let $\tau=\sum m_{i} \tau_{i}$ be a reducible character such that $\sigma-\tau$ is Syl $l_{p}$-vanishing of degree 0 .

(1) There is a unique $i$ such that $\tau_{i}$ is regular;

(2) Suppose $H$ has no component of type $B_{n}(2), C_{n}(2),{ }^{2} B_{2}(2), F_{4}(2),{ }^{2} F_{4}(2)$, $G_{2}(2), G_{2}(3),{ }^{2} G_{2}(3)$. If $\sigma$ is cuspidal then $\gamma(\tau)=\tau_{i}$, that is, $\tau_{j}$ does not belong to any Gelfand-Graev character for $j \neq i$; in addition, if $H=G L(n, q)$ then $\tau$ is irreducible and hence Syl $p_{p}$-equivalent to $\sigma$;

(3) $\gamma(\tau)$ is multiplicity free, that is, $m_{i}=1$ if $\tau_{i}$ is a constituent of some Gelfand-Graev character.

Proof. (1) follows by taking the inner product of $\sigma-\tau$ with the non-degenerate Gelfand-Graev character of $H$.

(2) By the way of contradiction, suppose that $\tau_{j}$ for $j \neq i$ is a constituent of a Gelfand-Graev character $\Gamma$ of $H$. By Lemma 3.5, there is a Levi subgroup $L$ of $H$ such that $\Gamma=\Gamma_{L}^{\# H}$. Note that $L \neq H$, as $(\tau, \Gamma)=\left(\tau_{i}, \Gamma\right)=1$. Since $\left(\tau_{j}, \Gamma_{L}^{\# H}\right)>0$, we have $\left(\sigma, \Gamma_{L}^{\# H}\right)>0$, which is false as $\sigma$ is cuspidal.

To prove the additional statement, recall that every irreducible character of $H=G L(n, q)$ is a constituent of some Gelfand-Graev character (Lemma 3.13). This implies $\gamma(\tau)=\tau$.

(3) Let $\tau_{i}$ is a constituent of a Gelfand-Graev character $\nu^{H}$. Suppose that $m_{i}>$ 1. Then $\left(\tau, \nu^{H}\right) \geq m_{i}>1$, and hence $\left(\sigma, \nu^{H}\right)>1$. Then $\left(\chi, \nu^{H}\right)>1$, which contradicts Lemma 3.1 . 
Lemma 4.4. Let $\chi, \chi_{1}, \ldots, \chi_{k} \in \operatorname{Irr} H$, and $\chi(1)<|H|_{p}$. Suppose that $\chi(g)=$ $\chi_{1}(g)+\cdots+\chi_{k}(g)$ for every $p$-singular element $g \in H$. Then all characters $\chi, \chi_{1}, \ldots, \chi_{k}$ belong to the same $p$-block.

Proof. Let $B$ be the block $\chi$ belongs to. By reordering the $\chi_{i}$ 's we can assume that $\chi_{1}, \ldots, \chi_{m} \in B$, and $\chi_{m+1}, \ldots, \chi_{k} \notin B$. By Feit [8, Ch. IV, Lemma 3.14], it follows that $\sigma:=\chi_{m+1}+\cdots+\chi_{k}$ is a $p$-vanishing character, and hence $\sigma(1)$ is a multiple of $|H|_{p}$. So $\sigma(1)=0$, and hence $\sigma=0$, as claimed.

4.2. The computational aspect. In Sections 5 and 9 we compute the $S y l_{p^{-}}$ vanishing characters $\psi$ of degree $|G|_{p}$ for some Chevalley groups $G$, whose character tables are known. Our main reference here is the algebra package CHEvIE [9], in particular, its MAPLE part. Recall that CHEVIE consists of a library of data and programs to deal with the generic character table of certain finite groups $G$ of Lie type of small rank. If $G$ is a such group, defined over the field $F_{q}$, the generic character table of $G$ depends on this parameter $q$. So, all the information stored in CHEVIE is expressed in the form of functions of $q$ : for instance, the degrees and the number of the irreducible characters as well as the number and the representatives of the conjugacy classes of $G$.

We keep the notation of the CHEVIE package for the irreducible characters and the conjugacy classes. In particular, CHEvIE partitions $\operatorname{Irr} G$ in sets, which we call CHEVIE sets and denote by $\mathbf{X}_{h}=\left\{\chi_{h}(k) \mid k \in I_{h}\right\}$ in this paper. Here $h$ is a natural number and $I_{h}$ is some parameter set, called the character parameter group (see the CHEVIE manual [9]). Furthermore, CHEVIE partitions the conjugacy classes of $G$ in sets $\mathbf{C}_{h}=\left\{C_{h}(k) \mid k \in I_{h}\right\}$, where, as before, $I_{h}$ is the corresponding parameter set. Note the classes belonging to the same set $\mathbf{C}_{h}$ have the same order.

To avoid misunderstanding, we emphasize that CHEvIE notation for parameters $k \in I_{h}$ for different groups is often not homogeneous, but we are forced to follow CHEviE. For instance, the set $I_{4}$ of $U(3, q)$ is parametrised by $(u, v)$, and the set $I_{10}$ of $S U(3, q)$, with $3 \mid(q+1)$, is parametrised by $(n, m)$.

It is essential for us that the characters in the same CHEviE set $\mathbf{X}_{h}$ are $S y l_{p^{-}}$ equivalent (but the converse is not true). This allows us, in performing computations with $S y l_{p}$-vanishing characters, to ignore the parameter $k$ that determines an individual character $\chi_{h}(k) \in \mathbf{X}_{h}$. By this reason we write $\chi_{h}$ to denote any character of $\mathbf{X}_{h}$ (to simplify the notation).

Our strategy involves three steps, that we describe as follows.

Procedure 4.5. In order to determine the set of all $S y l_{p}$-vanishing characters $\psi$ of a group $G$ with $\psi(1)=|G|_{p}$, we apply the following procedure:

(1) Compute some $S y l_{p}$-decompositions of the irreducible characters $\chi_{h}$ of $G$ (the results are given in the tables of Section 11). For $S y l_{p}$-decompositions we choose to compact the notation by writing $\mathbf{v}=\left(j_{1}, \cdots, j_{t}\right)$, especially in the tables. The case where $t=1$ means that $\chi_{h}$ and $\chi_{j_{1}}$ are $S y l_{p}$-equivalent. (This is reflected in the tables, see, for instance Table 11.2]3-A.) Note that we do not guarantee that our tables contain all $S y l_{p}$-decomposable irreducible characters, this is not necessary for our purposes. To simplify the notation, we denote by $\Delta_{G}$ the set of the indices $h$ corresponding to the irreducible characters $\chi_{h}$ of $G$ that have no known $S y l_{p}$-decomposition.

(2) Determine all $S y l_{p}$-vanishing characters of degree $|G|_{p}$ whose irreducible constituents $\chi_{j}$ are chosen with $j \in \Delta_{G}$. Note that by Lemma 3.1, every 
character $\psi$ has at least one regular character constituent (exactly one if $G$ has a unique non-degenerate Gelfand-Graev character). We use CHEvIE to identify the regular characters of $G$ in our list of $S y l_{p}$-decompositions. This allows us to assume that every regular constituent of $\psi$ has no known $S y l_{p}$-decomposition.

(3) If $\sum \chi_{i}$ is a subcharacter of a character $\psi$ obtained in step (2) and this subcharacter is also a $S y l_{p}$-decomposition of an irreducible character $\tau$ of $G$ (obtained in step (1)), then the substitution with $\tau$ in place of $\sum \chi_{i}$ yields a new $S y l_{p}$-vanishing character with a lower number of constituents. Repeating this, we arrive at $S y l_{p}$-vanishing characters that does not contain such subcharacters. In this way, we are able to list all the $S y l_{p}$-vanishing characters of $G$ of degree $|G|_{p}$.

Afterwards, we use the character table of $G$ in order to detect and rule out those $S y l_{p}$-vanishing characters that are not $p$-vanishing. In most cases we do this for groups with connected center. However, in the last step we try to only use elements that belong to the derived subgroup $G^{\prime}$ of $G$. In view of Proposition 6.2 this yields the result for groups with disconnected center.

Note that we have found quite a lot of $S y l_{p}$-decomposable characters, including regular and semisimple, and this hints that the use of them can organise $S y l_{p^{-}}$ vanishing characters in a more conceptual way.

Also, observe that every character $\chi_{h} \in \mathbf{X}_{h}$ occurs with the same multiplicity in a Gelfand-Graev character $\nu^{G}$ for a fixed $\nu \in \operatorname{Irr} U$. So, by Lemma 2.4, we have:

Lemma 4.6. Suppose that $G$ is with connected center. If some character $\chi_{h} \in \mathbf{X}_{h}$ is regular then so are all characters in $\mathbf{X}_{h}$. In addition, if $\chi$ is a Syl $p_{\text {-vanishing }}$ character of $G$ of degree $|G|_{p}$ then for any fixed $h$ there is at most one regular character from $\mathbf{X}_{h}$ occurring in $\chi$.

In the next sections we often use the following simple observation. Let $H$ be a finite reductive group and $G$ the subgroup generated by unipotent elements. Then $H / G$ is abelian. Suppose that $G$ is quasi-simple. Let $\chi$ be a $p$-vanishing character of $H$ of degree $|H|_{p}$. Then $\chi$ contains a one-dimensional constituent if and only if $\left.\chi\right|_{G}$ contains $1_{G}$. Therefore, $\chi \cdot \nu$ contains $1_{H}$ for some one-dimensional character $\nu$ of $H$.

\section{Chevalley groups of BN-Pair RANK 1}

Now we start dealing with Chevalley groups of rank 1: $S L(2, q), S U(3, q)$, ${ }^{2} B_{2}\left(q^{2}\right)=S z\left(q^{2}\right)$ with $q^{2}=2^{2 m+1}$, and ${ }^{2} G_{2}\left(q^{2}\right)$ with $q^{2}=3^{2 m+1}$.

In this section, as well as in Sections 7 and 9, we shall use the following notation for certain complex numbers:

$$
\begin{array}{llll}
\omega=\exp \left(\frac{2 \pi i}{3}\right), & \zeta_{1}=\exp \left(\frac{2 \pi i}{q-1}\right), & \xi_{1}=\exp \left(\frac{2 \pi i}{q+1}\right), & \xi_{2}=\exp \left(\frac{2 \pi i}{q^{2}+1}\right), \\
\xi_{3}=\exp \left(\frac{2 \pi i}{q^{3}+1}\right), & \varphi_{8}^{\prime}=\exp \left(\frac{2 \pi i}{q^{2}+\sqrt{2} q+1}\right), & \varphi_{12}^{\prime \prime}=\exp \left(\frac{2 \pi i}{q^{2}+\sqrt{3} q+1}\right) . &
\end{array}
$$

5.1. Groups $S L(2, q)$ and $G L(2, q)$. One observes that all irreducible characters of $G L(2, q)$ of the same degree are $S y l_{p}$-equivalent. Inspection of the character table of $G=S L(2, q)$ yields:

Lemma 5.1. Let $G=S L(2, q)$ and $\psi$ be a reducible character of degree $q$. Then $\psi$ is $S y l_{p}$-vanishing if and only if one of the following holds: 
(1) $\psi-1_{G}$ is an irreducible character;

(2) $\psi-1_{G}=\eta_{1}+\eta_{2}$, where $\eta_{1}, \eta_{2}$ are distinct characters of degree $(q-1) / 2$;

(3) $\psi=\tau+\eta$, where $\tau(1)=(q+1) / 2$ and $\eta(1)=(q-1) / 2$ and $\tau, \eta$ belong to distinct Weil representations of $G$.

In addition, $\psi$ is p-vanishing if and only if $\psi$ is Syl $_{p}$-vanishing and $\psi(z)=\psi(1)$ for all $z \in Z(G)$.

Remark 5.2. (i) The character $\psi-1_{G}$ in (1) and $\eta_{1}, \eta_{2}$ are cuspidal. (ii) The cases (2), (3) occur only for $q$ odd. (iii) The cases (3) cannot occur for $G=P S L(2, q)$. (iv) In (3) the characters $\tau, \eta$ are in distinct blocks.

Lemma 5.3. Let $H=G L(2, q)$. A reducible character $\psi$ of $H$ of degree $q$ is Syl $l_{p}$-vanishing if and only if $\psi=\lambda+\eta$, where $\lambda(1)=1$, and $\eta$ is an irreducible cuspidal character of degree $\eta(1)=q-1$. In this case, $\psi$ is p-vanishing if and only if $\operatorname{Ker}(\psi)=Z(H)$.

In CHEviE notation, $\psi=\chi_{1}\left(k_{1}\right) \cdot\left(1_{H}+\chi_{4}\left(k_{4}\right)\right)$, where $\chi_{1}\left(k_{1}\right)\left(k_{1} \in I_{1}\right)$ is a linear character of $H$ and $k_{4} \in I_{4}$ with $k_{4}=(q-1) k_{4}^{\prime}$ (see Tables 11.1-A,B,C,D).

5.2 . The unitary groups $H=U(3, q)$ and $G=S U(3, q)$. We first consider the groups $H=U(3, q)$. According to CHEvie, we partition $\operatorname{Irr} H$ into 8 sets $\mathbf{X}_{h}$, and the characters in $\mathbf{X}_{h}$ are parametrized by a corresponding set $I_{h}$ of parameters (see Table 11.2 1-A). Step (1) of Procedure 4.5 yields the $S y l_{p}$-decompositions of Table 11.2.1-B and $\Delta_{H}=\{1,2,6\}$. On step (2) we obtain a unique character $\psi_{1}=\chi_{1}+\chi_{2}+\chi_{2}^{\prime}+\chi_{6}$, where $\chi_{2}, \chi_{2}^{\prime} \in \mathbf{X}_{2}$ are not necessarily distinct. Finally, in step (3) we get all $S y l_{p}$-vanishing characters of $H$ of degree $|H|_{p}$ (see Table 11.2. 1-C). To determine the $p$-vanishing characters of $H$ of degree $|H|_{p}$, we use the character table of $H$.

Lemma 5.4. Let $H=U(3, q)$. Then $H$ admits a reducible p-vanishing character $\psi$ of degree $|H|_{p}$ if and only if $3 \mid(q+1)$. In this case, there are exactly $q+1$ characters $\psi$, all of type

$$
\psi=\chi_{1}(u) \cdot\left(1_{H}+\chi_{2}(a)+\chi_{2}(2 a)+\chi_{6}(a, 2 a, 3 a)\right),
$$

where $a=(q+1) / 3$ and $\chi_{1}(u)$ is a linear character of $H$.

Proof. Let $\psi$ be a $S y l_{p}$-vanishing character of $H$ of degree $|H|_{p}=q^{3}$ and suppose that $\psi$ is also $p$-vanishing. By Lemma 2.1, all the constituents of $\psi$ belong to the same block. So it suffices to look at the classes $C_{5}(k, l)$, where $(k, l) \in \mathbb{Z}_{q+1} \times \mathbb{Z}_{q+1}$ and $k \neq l$. We have one of the following cases, modulo linear characters of $H$ :

(1) Take $\psi_{1}=1_{H}+\chi_{2}\left(u_{2}\right)+\chi_{2}\left(u_{2}^{\prime}\right)+\chi_{6}\left(u_{6}, v_{6}, w_{6}\right)$. As all the summands are in the same block, it follows that $q+1$ divides $3 u_{2}, 3 u_{2}^{\prime}$ and $u_{6}+v_{6}+w_{6}$. We obtain

$$
1+\xi_{1}^{-(k-l) u_{2}}+\xi_{1}^{-(k-l) u_{2}^{\prime}}=\xi_{1}^{(k-l)\left(u_{6}+v_{6}\right)}+\xi_{1}^{-(k-l) v_{6}}+\xi_{1}^{-(k-l) u_{6}} \text {. }
$$

This holds for every $k, l$ with $k \neq l$. This implies that 3 divides $q+1$, and, by symmetry, $u_{2}=a, u_{2}^{\prime}=2 a$ and $\left(u_{6}, v_{6}, w_{6}\right)=(a, 2 a, 3 a)$, with $a=(q+1) / 3$.

(2) Take $\psi_{2}=\chi_{2}(0)+\chi_{4}\left(u_{4}, v_{4}\right)+\chi_{6}\left(u_{6}, v_{6}, w_{6}\right)$, with $q+1$ dividing both $2 u_{4}+v_{4}$ and $u_{6}+v_{6}+w_{6}$. We obtain

$$
1+\xi_{1}^{-(k-l) u_{4}}+\xi_{1}^{2(k-l) u_{4}}=\xi_{1}^{(k-l)\left(u_{6}+v_{6}\right)}+\xi_{1}^{-(k-l) v_{6}}+\xi_{1}^{-(k-l) u_{6}} .
$$

We have the following possibilities: 
(a) Either $u_{6}=0$ or $v_{6}=0$ : this implies $u_{4}=0$ and $v_{4}=0$, a contradiction with the conditions on the parameters.

(b) $u_{6}+v_{6}=0$ : again we obtain $u_{4}=v_{4}=0$.

(3) Take $\psi_{3}=\chi_{2}(0)+\chi_{5}\left(u_{5}, v_{5}\right)$ : we obtain $\xi_{1}^{k\left(u_{5}+v_{5}\right)+l u_{5}}=1$, which implies $u_{5}=v_{5}=0$, a contradiction with the conditions on the parameters.

Next, let $G=S U(3, q)$, where $3 \nmid(q+1)$. This case is very similar to the case $U(3, q)$. We list the the relevant informations in Tables 11.2 2-A,B,C. Thus, it can be easily proved the following.

Lemma 5.5. Let $G=S U(3, q)$, where $3 \nmid(q+1)$. Then $G$ admit no reducible p-vanishing character of degree $|G|_{p}$.

Finally, let $G=S U(3, q)$ with $3 \mid(q+1)$. Applying Procedure 4.5 we obtain on step (1) the $S y l_{p}$-decompositions of Table 11.2.3-B and $\Delta_{G}=\{1,2,7,8,9\}$. Step (2) yields only one character $\psi_{1}=\chi_{1}+2 \chi_{2}+\chi_{7}+\chi_{8}+\chi_{9}$. With step (3) we obtain all $S y l_{p}$-vanishing characters of $G$ of degree $|G|_{p}$ (see Table 11.2.3-C).

Lemma 5.6. Let $G=S U(3, q)$, with $3 \mid(q+1)$. Then $G$ admits a unique reducible p-vanishing character $\psi$ of degree $|G|_{p}$, namely $\psi=1_{G}+2 \chi_{2}+\chi_{7}+\chi_{8}+\chi_{9}$.

Proof. The cases $q=2,5,8$ can be easily dealt with magma. So, assume $q \geq$ 11. Applying Lemma 2.1, we easily rule out all the options where the irreducible constituents of $\psi$ are not in the same block. So, we have to study only the following five cases:

(1) $\psi_{1}=\chi_{1}+2 \chi_{2}+\chi_{7}+\chi_{8}+\chi_{9}$ : this character is $p$-vanishing.

(2) $\psi_{2}=\chi_{1}+2 \chi_{2}+\chi_{10}(n, m)$ : on the class $C_{7}(1)$ we obtain $\xi_{1}^{n-2 m}+\xi_{1}^{m-2 n}+$ $\xi_{1}^{n+m}=3$. So, $\xi_{1}^{n-2 m}=\xi_{1}^{m-2 n}=\xi_{1}^{n+m}=1$ and hence $(m, n)=\left(\frac{q+1}{3}, \frac{2(q+1)}{3}\right)$, $\left(\frac{2(q+1)}{3}, \frac{q+1}{3}\right)$. But, for this choice of the parameters, $\chi_{10}=\chi_{7}+\chi_{8}+\chi_{9}$.

(3) $\psi_{21}=\chi_{2}+\chi_{4}(k)+\chi_{7}+\chi_{8}+\chi_{9}$ : on the class $C_{7}(1)$ we obtain $\xi_{1}^{k}+\xi^{-2 k}=2$ and so $\xi_{1}^{k}=1$, a contradiction.

(4) $\psi_{22}=\chi_{2}+\chi_{4}(k)+\chi_{10}(n, m)$ : on the classes $C_{7}(a)(a=1,2,3)$ we obtain $1+\xi_{1}^{a k}+\xi_{1}^{-2 a k}=\xi_{1}^{a(n-2 m)}+\xi_{1}^{a(m-2 n)}+\xi_{1}^{a(n+m)}$. It follows that $(m, n)=$ $\left(\frac{q+1}{3}, \frac{2(q+1)}{3}\right),\left(\frac{2(q+1)}{3}, \frac{q+1}{3}\right)$. So $(q+1) \mid k$, a contradiction.

(5) $\psi_{23}=\chi_{2}+\chi_{5}(n)$ : on the class $C_{7}(1)$ we obtain $\xi_{1}^{n}=1$, i.e. $(q+1) \mid n$, a contradiction with the conditions on the parameter set $I_{5}$.

Thus, $\psi_{1}$ is the only reducible $p$-vanishing character of $G$.

5.3. Groups ${ }^{2} B_{2}\left(q^{2}\right)$ and ${ }^{2} G_{2}\left(q^{2}\right)$.

Lemma 5.7. Let $G={ }^{2} B_{2}\left(q^{2}\right), q^{2}=2^{2 m+1}$. Then $G$ admits exactly $2^{m-1}\left(2^{m}+1\right)$ reducible p-vanishing characters $\psi$ of degree $|G|_{p}$, all of type

$$
\psi=1_{G}+\chi_{2}+\chi_{3}+\chi_{6}(k) .
$$

Proof. We apply Procedure 4.5, In step (1) we obtain the $S y l_{p}$-decompositions of Table 11.3. B and $\Delta_{G}=\{1,2,3,6\}$. Step (2) produces a unique character $\psi_{1}=$ $\chi_{1}+\chi_{2}+\chi_{3}+\chi_{6}$. Finally, with step (3) we are able to list all the $S y l_{p}$-vanishing characters of $G$ of degree $|G|_{p}$ (see Table 11.3.C). As every element of $G$ is either semisimple or unipotent, every $S y l_{p}$-vanishing character is $p$-vanishing. 
Let $G={ }^{2} G_{2}\left(q^{2}\right)$, with $q^{2}=3^{2 m+1}, m \geq 0$. In order to reduce the number of the characters $\chi_{h}$ we have to consider, it is easier to work first only with the classes $C_{1}, C_{2}, C_{5}$. We obtain the decompositions listed in Table 11.4 B. These decompositions allow us to work only with the characters $\chi_{h}$, where $h \in\{1,4,5,7,9,12,13,14\}$. Working only with these constituents, we obtain a unique character of degree $|G|_{p}$ vanishing on the classes $C_{2}$ and $C_{5}: \theta=\chi_{1}+2 \chi_{5}+2 \chi_{7}+\chi_{14}$. Next we apply the decompositions of Table 11.4. B to the character $\theta$, to verify if the resulting character is $S y l_{p}$-vanishing. Finally, we are able to list all $S y l_{p}$-vanishing characters of $G$ of degree $|G|_{p}$ (see Table 11.4 $\mathrm{C}$ ).

Lemma 5.8. Let $G={ }^{2} G_{2}\left(q^{2}\right)$, with $q^{2}=3^{2 m+1}$. Then $G$ admits exactly $3^{m}\left(3^{m}+\right.$ $1) / 2$ reducible $p$-vanishing characters $\psi$ of degree $|G|_{p}$, all of type

$$
\psi=1_{G}+\chi_{3}+\chi_{5}+\chi_{6}+\chi_{7}+\chi_{14}(k) .
$$

Proof. Direct inspection of the character table shows that every $S y l_{p}$-vanishing character of $G$ of degree $|G|_{p}$ is $p$-vanishing.

Observe that the group $G={ }^{2} G_{2}(3)^{\prime}$ has a unique reducible 3-vanishing character of degree $|G|_{3}: \phi=\chi_{1}+\chi_{6}$ (with the notation of [3]). On the other hand, it has 16 reducible 3 -vanishing characters of degree $3|G|_{3}$.

(1) Ten characters of shape $\phi_{1}=a \chi_{7}+b \chi_{8}+c \chi_{9}$, where $0 \leq a \leq b \leq c \leq 3$ and $a+b+c=3$.

(2) Six characters of shape $\phi_{2}=\chi_{1}+\chi_{6}+d \chi_{7}+e \chi_{8}+f \chi_{9}$, where $0 \leq d \leq$ $e \leq f \leq 2$ and $d+e+f=2$.

This can be easily proved looking at the character table of $G$.

\section{6. $S y l_{p}$-VANishing CHARACTERS AND LEVI SUbGROUPS}

Let $\mathbf{H}$ be a reductive group with Frobenius endomorphism $F r$ and $\mathbf{G}$ the maximal semisimple subgroup of $\mathbf{H}$. Set $H=\mathbf{H}^{F r}$ and $G=\mathbf{G}^{F r}$.

Lemma 6.1 (15, Proposition p. 162]). Let $\tau$ be an irreducible character of $H$. Then $\left.\tau\right|_{G}$ is multiplicity free.

Proposition 6.2. Let $G, H$ be as above. Let $\chi$ be a p-vanishing character of $G$. Then $\chi$ is $H$-invariant and extends to a Syl ${ }_{p}$-vanishing character $\chi^{\prime}$ of $H$ such that $\left.\chi^{\prime}\right|_{G}=\chi$.

Proof. Note that $\chi$ is an integral linear combination of the characters of projective indecomposable modules, and every of them extends to $H$ (see [24, Lemma 3.14]). It follows that $\chi$ extends to a generalized $p$-vanishing character $\phi$ of $H$ such that $\left.\phi\right|_{G}=\chi$. This implies that $\chi$ is $H$-invariant. Next, we shall modify $\phi$ to obtain an ordinary $S y l_{p}$-vanishing character.

For $\sigma \in \operatorname{Irr} G$ denote by $\tilde{\sigma}$ the sum of the distinct $H$-conjugates of $\sigma$. As $\chi$ is $H$-invariant, we can write $\chi=\sum m(\tilde{\sigma}) \tilde{\sigma}$ for some integers $m(\tilde{\sigma}) \geq 0$, with the sum over the minimal $H$-invariant characters of $G$.

Let $\tau \in \operatorname{Irr} H$. By Clifford's theorem, $\left.\tau\right|_{G}=d_{\tau} \tilde{\sigma}$ for some integer $d_{\tau}>0$. By Lemma 6.1, $d_{\tau}=1$. Set $\operatorname{Irr}_{\tilde{\sigma}} H=\left\{\tau \in \operatorname{Irr} H:\left.\tau\right|_{G}=\tilde{\sigma}\right\}$. Note that if $\tau_{1}, \tau_{2} \in \operatorname{Irr}_{\tilde{\sigma}} H$ then $\left.\tau_{1}\right|_{G}=\left.\tau_{2}\right|_{G}=\tilde{\sigma}$. Then we have

$$
\phi=\sum_{\tilde{\sigma}} \sum_{\tau \in \operatorname{Ir} \tilde{\sigma} H} m(\tau) \tau
$$


where the first sum runs over the minimal $H$-invariant characters of $G$. Therefore, the multiplicity of $\tilde{\sigma}$ in $\phi$ equals $m(\tilde{\sigma})=\sum_{\tau \in \operatorname{Irr} \tilde{\sigma} H} m(\tau)$. Let $a_{\tilde{\sigma}}$ (resp., $b_{\tilde{\sigma}}$ ) be the sum of all positive (resp., negative) coefficients $m(\tau)$ occurring in this sum. Then $a_{\tilde{\sigma}} \geq b_{\tilde{\sigma}}$ as $m(\tilde{\sigma}) \geq 0$. Therefore, $\left.\sum_{\tau \in \operatorname{Irr} \tilde{\sigma} H} m(\tau) \tau\right|_{G}=\left.\left(a_{\tilde{\sigma}}-b_{\tilde{\sigma}}\right) \tau^{\prime}\right|_{G}$ for any $\tau^{\prime} \in \operatorname{Irr}_{\tilde{\sigma}} H$, and $\left(a_{\tilde{\sigma}}-b_{\tilde{\sigma}}\right) \cdot \tau^{\prime}$ is an ordinary character of $H$. So we replace every generalized character $\sum_{\tau \in \operatorname{Irr}_{\tilde{\sigma}} H} m(\tau) \tau$ by $\left(a_{\tilde{\sigma}}-b_{\tilde{\sigma}}\right) \cdot \tau^{\prime}$ to obtain a required modification $\chi^{\prime}$ of $\phi$.

Remark 6.3. Proposition 6.2 is not true for $S y l_{p}$-vanishing characters. Indeed, the characters of $G=S L(2, q)$ in the item (3) of Lemma 5.1 do not extend to $G L(2, q)$.

Note that $H$ is a group with split BN-pair, see [16, 24.10] or [2, p. 50]. So the Steinberg character can be defined for $H$ as in [6, 66.35 and 71.2]. This is of degree $|H|_{p}$, and hence of defect 0 .

Lemma 6.4. Let $\chi$ be a Syl ${ }_{p}$-vanishing character of $H$. Let $L$ be a Levi subgroup of some parabolic subgroup $P$ of $H$.

(1) If $\chi \in \operatorname{Irr} H$ then $\chi(1)=|H|_{p}$, and $\left.\chi\right|_{G}$ is the Steinberg character of $G$.

(2) Suppose that the truncation $\bar{\chi}_{L}$ is irreducible. Then $\chi(1)=|H|_{p}$.

(3) If $\chi \in \operatorname{Irr} H$ is of defect 0 then the truncation $\bar{\chi}_{L}$ is irreducible of defect 0.

Proof. (1) is well known. (Indeed, $\chi(1)$ is divisible by $|H|_{p}$, so $\chi$ is of defect 0 , and hence $\chi(\bmod p)$ is irreducible. The $p$-modular irreducible characters of $H$ remain irreducible under restriction to $G$ (cf. [24, 3.14]), and hence have degree at most $|G|_{p}=|H|_{p}$ (see [6, 72.19] or [22, Corollary of Theorem 46]). This implies (1).)

(2) As $\bar{\chi}_{L}$ is irreducible, it is of degree $|L|_{p}$ by (1). As $|H: P|$ is coprime to $p$, the claim follows from Lemma 2.5 .

(3) As $\chi$ is of defect 0 , the reduction of $\chi$ modulo $p$ is irreducible and the corresponding module $M$ is projective. By Smith's theorem [6, 72.32], the HarishChandra restriction $\bar{M}_{L}$ of $M$ to $L$ is irreducible and, moreover, projective. It is known that $\bar{\chi}_{L}$ is the character of $\bar{M}_{L}$, cf. [24, Lemma 3.3]. It follows that $\bar{\chi}_{L}$ is irreducible of defect 0 .

Lemma 6.5. Let $\phi$ be a defect 0 irreducible character of $H$, and $L$ a Levi subgroup of $H$. Then $\phi=S t$ if and only if $\bar{\phi}_{L}=S t_{L}$.

Proof. The "only if" part is well known, see for instance [7, p. 72]. For the "if" part suppose that $\bar{\phi}_{L}=S t_{L}$. First we prove the lemma in the case where $L=T \subset B$.

Recall that $\phi(1)=|H|_{p}$ [6, 72.20], equivalently, $\bar{\phi}_{T}(1)=1$ (Lemma 2.5). Suppose that $\left(\phi, 1_{B}\right)=1$. By Lemma 6.4, $\left.S t_{H}\right|_{G}=S t_{G}$ and $\left.\phi\right|_{G}=S t_{G}$. For every $h \in H$ we have $\phi(h)=S t_{H}(h) \cdot \mu(h)$ for some complex number $\mu(h)$. It follows that the function $\mu$ is multiplicative (view $\phi$ and $S t_{H}$ as representations rather than characters). As $H$ is a finite group, $\mu$ is a representation. Therefore, $\phi=S t_{H} \cdot \mu$. As $U \subset G$, we have $\mu(U)=1$. (Usually $U$ is in the kernel of every linear character of $H$ but there are a few exceptions.) Let $\phi=S t \cdot \mu$. Then $1=\left(\left.\phi\right|_{B}, 1_{B}\right)=$ $\left(\left.\left.S t\right|_{B} \cdot \mu\right|_{B}, 1_{B}\right)=\left(\left.S t\right|_{B},\left.\mu^{*}\right|_{B} \cdot 1_{B}\right)=\left(\left.S t\right|_{B},\left.\mu^{*}\right|_{B}\right)$, where $\mu^{*}$ is the complex conjugate to $\mu$. By the above, $\left.\mu^{*}\right|_{B}=1_{B}$. We show that $\mu^{*}=1_{H}$.

Let $H_{0}$ be the subgroup generated by the unipotent elements in $H$. Then $H / H_{0}$ is a $p^{\prime}$-group. As $H_{0} B$ contains $B$, this is a parabolic subgroup of $H$, and $H_{0} B=H$ as every parabolic subgroup coincides with its normalizer [6, 65.19]. It follows that 
the mapping $\left.\mu \rightarrow \mu\right|_{B}$ yields an injective mapping from $\operatorname{Irr} H / H_{0}$ to $\operatorname{Irr} B / B^{\prime}$, where $B^{\prime}$ is a derived subgroup of $B$. Therefore, $\left.\mu\right|_{B}=1_{B}$ implies $\mu=1_{H}$.

Let $L \neq T$ and let $\eta=\bar{\phi}_{L}$. By transitivity of truncation, $\bar{\phi}_{T}=\bar{\eta}_{T}$. By the above, $\phi=S t$ is equivalent to $\bar{\phi}_{T}=1_{T}$, and $\bar{\eta}_{T}=1_{T}$ is equivalent to $\eta=S t_{L}$. So the result follows.

We keep the notation $H, G$ as in the beginning of the section, and assume that $\mathbf{G}$ is simply connected. In what follows we normally view $H$ and $G$ as groups with a split $B N$-pair defined by a Borel subgroup $B$ and a subgroup $N$ satisfying certain conditions, see [6, §65]. We set $T=N \cap B$ and $W=N / T$ (which is called the Weyl group). We denote by $\ell$ the BN-pair rank of $H$. (This is not the same as the rank of $\mathbf{H}$.) Set $I_{\ell}=\{1, \ldots, \ell\}$. The standard parabolic subgroups of $H$ are those containing $B$. They form a lattice isomorphic to the lattice of the subsets of $I_{\ell}$. We denote by $P_{J}$ the standard parabolic subgroup corresponding to a subset $J$ of $I_{\ell}$ (and $B$ corresponds to the empty set). Let $U_{J}:=O_{p}\left(P_{J}\right)$ be the unipotent radical of $P_{J}$. Then $P_{J}=U_{J} \rtimes L_{J}$ for some subgroup $L_{J}$ called the Levi subgroup of $P_{J}$. The Levi subgroups are conjugate in $P_{J}$, and it is possible to choose $L_{J}$ to contain $T$. (This is always assumed below.) If $K \subseteq J$, then $P_{K} \cap L_{J}$ is a standard parabolic subgroup of $L_{J}$ containing the Borel subgroup $B \cap L_{J}$ of $L_{J}$. Furthermore, $O_{p}\left(P_{K} \cap L_{J}\right)=U_{K} \cap L_{J}, P_{K} \cap L_{J}=\left(U_{K} \cap L_{J}\right) \rtimes L_{K}$ and $U_{K}=U_{J}\left(U_{K} \cap L_{J}\right)$. In turn, $L_{J}$ is a group with BN-pair whose Weyl group $W_{J}$ is a subgroup of $W$. The Weyl group $W$ has a unique (up to equivalence) irreducible representation $\rho$ of degree $\ell$ called the reflection representation, and $\varepsilon:=\operatorname{det} \circ \rho$ is called the sign character of $W$.

Let $P_{J}=L_{J} U_{J}$, where $L_{J}$ is a Levi subgroup of $P_{J}$. Denote by $G_{J}$ the normal subgroup of $L_{J}$ generated by the unipotent elements of $L_{J}$. Then $L_{J}=G_{J} T$. Set $B_{J}=B \cap G_{J}$ and $T_{J}=T_{0} \cap G_{J}$. Then $B_{J}$ is a Borel subgroup of $G_{J}$ and $B_{J}=\left(U \cap L_{J}\right) \rtimes T_{J}$. (Note that $B_{J}$ differs from $B \cap L_{J}$, which is a Borel subgroup of $L_{J}$.)

Recall that we call $G$ a Chevalley group if $\mathbf{G}$ is simple and simply connected.

In Section 5 we have obtained the following:

Proposition 6.6. Let $G$ be of BN-pair rank 1. Then $G$ admits a reducible $p$ vanishing character $\chi$ of degree $|G|_{p}$, unless $G=S U(3, q)$ with $(q+1,3)=1$. Furthermore, $\left(\chi, 1_{G}\right)=1$, and some constituent of $\chi$ is a cuspidal character of $G$.

Proof. The result follows from Lemmas 5.1, 5.5, 5.6, 5.7 and 5.8,

In order to deal with groups of higher rank, we establish some properties of $S y l_{p}$-vanishing characters.

Lemma 6.7. Suppose that $G=H$. Let $I_{\ell}=J \cup J^{\prime}$ be the union of two subsets $J, J^{\prime}$. Then $T=T_{J} T_{J^{\prime}}$.

Proof. The result follows from [11, Theorem 2.4.7(a)].

Lemma 6.8. Let $\chi$ be an arbitrary character of $H$, and let $\beta_{J}$ be the truncation of $\bar{\chi}_{L_{J}}$ to $T$ (so $\beta_{J}$ is a character of $T$ ).

(1) $\beta_{J}=\bar{\chi}_{T}$.

(2) Set $\lambda=\left.\bar{\chi}_{L_{J}}\right|_{G_{J}}$. Then $\bar{\lambda}_{T_{J}}=\left.\beta_{J}\right|_{T_{J}}$. 
Proof. (1) follows from the transitivity of truncation.

(2) By the definition of truncation, for all $t \in T$ and $g \in G_{J}$ we have

$$
\beta_{J}(t)=\frac{1}{|U|} \sum_{u \in U} \chi(u t) \text { and } \lambda(g)=\frac{1}{\left|U_{J}\right|} \sum_{v \in U_{J}} \chi(v g) .
$$

So, for all $x \in T_{J}$,

$$
\begin{gathered}
\left(\bar{\lambda}_{T_{J}}\right)(x)=\frac{1}{\left|U \cap L_{J}\right|} \sum_{y \in U \cap L_{J}} \lambda(y x)= \\
=\frac{1}{\left|U \cap L_{J}\right| \cdot\left|U_{J}\right|} \sum_{y \in U \cap L_{J}} \sum_{v \in U_{J}} \chi(v y x)=\frac{1}{|U|} \sum_{u \in U} \chi(u x)=\beta_{J}(x),
\end{gathered}
$$

using the fact that $U=U_{J}\left(U \cap L_{J}\right)$.

Lemma 6.9. Assume $G=H$. Let $I_{\ell}=J \cup J^{\prime}$ be the union of two subsets $J, J^{\prime}$. Let $\chi$ be a Syl $l_{p}$-vanishing character of $G$. Suppose that $\bar{\chi}_{L_{J}}$ and $\bar{\chi}_{L_{J^{\prime}}}$ are irreducible. Then $\bar{\chi}_{T}=1_{T}$.

Proof. Let $\eta=\left.\left(\bar{\chi}_{L_{J}}\right)\right|_{G_{J}}$ and $\eta^{\prime}=\left.\left(\bar{\chi}_{L_{J^{\prime}}}\right)\right|_{G_{J^{\prime}}}$. Then $\eta=S t_{G_{J}}$ and $\eta^{\prime}=S t_{G_{J^{\prime}}}$ (Lemma 6.4(1) and (2)). By Lemma 6.5, the truncation of $\eta$ to $T_{J}$ is $1_{T_{J}}$ and the truncation of $\eta^{\prime}$ to $T_{J^{\prime}}$ is $1_{T_{J^{\prime}}}$. By Lemma 6.8, $\left.\bar{\chi}_{T}\right|_{T_{J}}=1_{T_{J}}$ and $\left.\bar{\chi}_{T}\right|_{T_{J^{\prime}}}=1_{T_{J^{\prime}}}$. Now Lemma 6.7 yields the result.

The following result is our primary target; this allows us to use classical results (quoted in Lemmas 6.13 and 6.14) on irreducible constituents of of $1_{B}^{G}$.

Proposition 6.10. Let $G=H$ be and $\chi$ be a p-vanishing character of $G$ of degree $|G|_{p}$. Then $\left(\chi, 1_{B}^{G}\right)=\left(1_{B},\left.\chi\right|_{B}\right)=1$ (equivalently, $\left.\bar{\chi}_{T}=1_{T}\right)$.

Proof. The first equality is the Frobenius reciprocity. The second equality is equivalent to saying that $\bar{\chi}_{T}=1_{T}$. We use induction on the BN-pair rank of $G$. By Proposition 6.6, the proposition is true for groups of rank 1. Let $I_{\ell}=J \cup J^{\prime}$ be the union of two subsets $J, J^{\prime}$. Then $\eta:=\left.\bar{\chi}_{L_{J}}\right|_{G_{J}}\left(\right.$ resp., $\left.\eta^{\prime}:=\left.\bar{\chi}_{L_{J^{\prime}}}\right|_{G_{J^{\prime}}}\right)$ is a $p$-vanishing character of $G_{J}$ (resp., $G_{J^{\prime}}$ ) of degree $\left|G_{J}\right|_{p}$ (resp., $\left|G_{J^{\prime}}\right|_{p}$ ), see Lemma 2.5. By induction, the proposition is true for $G_{J}$ and $G_{J^{\prime}}$, so $\bar{\eta}_{T_{J}}=1_{T_{J}}$ and $\bar{\eta}_{T_{J^{\prime}}}=1_{T_{J^{\prime}}}$. By Lemma 6.8, $\bar{\eta}_{T_{J}}$ coincides with $\left.\bar{\chi}_{T}\right|_{T_{J}}\left(\right.$ as $L$ contains $T$ ). Therefore, $\left.\bar{\chi}_{T}\right|_{T_{J}}=1_{T_{J}}$ and $\left.\bar{\chi}_{T}\right|_{T_{J^{\prime}}}=1_{T_{J^{\prime}}}$. Now the result follows from Lemma 6.7.

Corollary 6.11. Assume $G=H$. Let $\chi$ be a $S y l_{p}$-vanishing character of $G$ such that $\bar{\chi}_{T}=1_{T}$, and $L$ a Levi subgroup of $G$.

(1) If $\bar{\chi}_{L}$ is irreducible of defect 0 , then $\bar{\chi}_{L}=S t_{L}$.

(2) If $L$ is not solvable and $\bar{\chi}_{L}$ has a 1-dimensional constituent $\nu$, then $\nu=1_{L}$.

Proof. (1) By transitivity of Harish-Chandra restriction, the truncation of $\bar{\chi}_{L}$ to $T$ coincides with $1_{T}$. So the claim follows from Lemma 6.5 applied to $L$ in place of $G$ and $T$ in place of $L$.

(2) Since the Borel subgroup of $L$ is $(U \cap L) T$, by the definition of truncation, $\bar{\nu}_{T}=\left.\left(\left.\nu\right|_{U \cap L}, 1_{U \cap L}\right) \cdot \nu\right|_{T}$. Hence, $\left.\nu\right|_{T}=1_{T}$. On the other hand, let $L_{u}$ be the subgroup of $L$ generated by the unipotent elements of $L$. Then, one easily observes that $L=L_{u} \cdot T$. Since $\nu$ is a linear character of $L$, the derived subgroup of $L$ coincides with $L_{u}$. It follows that $\left.\nu\right|_{L_{u}}=1_{L_{u}}$, so the result follows. 
It is known that the blocks of non-zero defect of a finite reductive group $H$ are in bijection with the elements of $Z(H)$, see [13, §8.7]; this is deduced from the fact that every such block has defect $|U|$ and $C_{H}(U)=Z(H)$. The following result shows that if a $p$-vanishing character of degree $|G|_{p}$ is in the principal $p$-block of $G$, then the Harish-Chandra reduction $\bar{\chi}_{L}$ of $\chi$ to every Levi subgroup $L$ belongs to the principal $p$-block of $L$.

Observe that Proposition 6.10 is a special case (for $L=T$ ) of the following result.

Corollary 6.12. Let $G=H$ and $L$ a Levi subgroup of $G$. Let $\chi$ be a p-vanishing character of $G$ of degree $|G|_{p}$. Then $Z(L)$ is in the kernel of $\bar{\chi}_{L}$.

Proof. As $Z(L)$ is contained in a Borel subgroup $B_{L}$ of $L$, it is contained in a maximal torus $T_{L}$ of $B_{L}$. However, $T_{L}$ coincides with a maximal torus $T$ of a Borel subgroup $B$ of $G$. So $Z(L) \subset T$. By Proposition 6.10, $1_{Z(L)}$ is a constituent of $\left.\bar{\chi}_{L}\right|_{Z(L)}$. Therefore, there is an irreducible constituent $\tau$, say, of $\bar{\chi}_{L}$ such that $\left.\tau\right|_{Z(L)}=\tau(1) \cdot 1_{Z(L)}$. In other words, $Z(L)$ is in the kernel of $\tau$.

Suppose that the lemma is false. Then $Z(L)$ is not in the kernel of some irreducible constituent $\tau^{\prime}$, say, of $\bar{\chi}_{L}$. Therefore, $\tau^{\prime}$ and $\tau$ belong to distinct $p$-blocks. As $Z(L) \subset T$, it follows that $(|Z(L)|, p)=1$. This is not the case by Lemma 2.1] as $\bar{\chi}_{L}$ is $p$-vanishing.

The following lemma is one of standard results on representations of groups with $B N$-pair, see [6. 68.24, pp. 683 - 684]. Recall that the Weyl group $W$ of a group $H$ with BN-pair has a natural representation as a linear group generated by reflections 6. 64.29]. The determinant mapping $w \rightarrow \operatorname{det} w(w \in W)$ of this representation yields a one-dimensional representation of $W$ called the sign representation. In the statement below the character of the sign representation is denoted by $\varepsilon$. For a subgroup $X$ of $W$ set $\varepsilon_{X}=\left.\varepsilon\right|_{X}$.

Lemma 6.13. Let $H$ be a finite reductive group viewed as a group with BN-pair of rank $\ell, B$ a Borel subgroup of $H$ and $W$ the Weyl group of the BN-pair.

(1) There exists a bijection $\lambda \mapsto \chi_{\lambda}$ between $\operatorname{Irr} W$ and the set $\{\chi \in \operatorname{Irr} H$ : $\left.\left(\chi, 1_{B}^{H}\right)>0\right\}$ such that $\chi_{1_{W}}=1_{H}$ and $\chi_{\varepsilon}=$ St. In addition, $\left(\chi_{\lambda}, 1_{B}^{H}\right)=$ $\lambda(1)$.

(2) For each subset $J$ of $I_{\ell}=\{1, \ldots, \ell\}$, let $W_{J}$ and $P_{J}$ be the corresponding subgroups of $W$ and $H$, respectively. Then

$$
\left(\chi_{\lambda}, 1_{P_{J}}^{H}\right)=\left(\lambda, 1_{W_{J}}^{W}\right)=\left(\left.\lambda\right|_{W_{J}}, 1_{W_{J}}\right) .
$$

In particular, $1_{B}^{H}=\sum_{\lambda \in \operatorname{Irr} W} \lambda(1) \cdot \chi_{\lambda}$.

(3) [6, 70.24] $\left(\chi_{\lambda}, S t_{L_{J}}^{\# H}\right)=\left(\lambda, \varepsilon_{W_{J}}^{W}\right)=\left(\left.\lambda\right|_{W_{J}}, \varepsilon_{W_{J}}\right)$.

Lemma 6.14 (6, (68.26)]). Let $W$ be in Lemma 6.13; assume that $W$ is not a direct product of proper subgroups. Then each irreducible character $\lambda$ of $\operatorname{Irr} W$ is uniquely determined by the multiplicities $\left\{\left(\lambda, 1_{W_{J}}^{W}\right): J \subseteq I_{\ell}\right\}$, with the following exceptions:

(1) the characters of degree 2 if $W$ is the dihedral group of order 12 or 16 ;

(2) the two characters of degree $2^{9}$ if $W$ is of type $E_{7}$;

(3) the four characters of degree $2^{12}$ if $W$ is of type $E_{8}$.

Corollary 6.15. Keep notation of Lemma 6.13 and assumption of Lemma 6.14, 
(1) Each irreducible character $\lambda$ of $\operatorname{Irr} W$ is uniquely determined by the multiplicities $\left\{\left(\lambda, \varepsilon_{W_{J}}^{W}\right): J \subseteq I_{\ell}\right\}$, with the exceptions (1),(2),(3) in Lemma 6.14 .

(2) The character $\chi_{\lambda}$ is uniquely determined by the multiplicities $\left\{\left(\chi_{\lambda}, 1_{P_{J}}^{H}\right)\right.$ : $\left.J \subseteq I_{\ell}\right\}$ as well as the multiplicities $\left\{\left(\chi_{\lambda}, S t_{L_{J}}^{\# H}\right): J \subseteq I_{\ell}\right\}$, unless $\lambda$ is listed in items (1), (2), (3) of Lemma 6.14.

Proof. (1) We have $\left(\lambda, \varepsilon_{W_{J}}^{W}\right)=\left(\lambda,\left(\left.\varepsilon\right|_{W_{J}}\right)^{W}\right)=\left(\lambda, 1_{W_{J}}^{W} \cdot \varepsilon\right)=\left(\lambda \cdot \varepsilon, 1_{W_{J}}^{W}\right)$. By Lemma 6.14, $\lambda \cdot \varepsilon$ is determined by the multiplicities $\left\{\left(\lambda \cdot \varepsilon, 1_{W_{J}}^{W}\right): J \subseteq I_{\ell}\right\}$, so $\lambda$ is determined by $\left\{\left(\lambda, \varepsilon_{W_{J}}^{W}\right): J \subseteq I_{\ell}\right\}$.

(2) follows from Lemmas 6.13 and 6.14, and statement (1) of this corollary.

The following lemma is a next important step in our analysis, as this settles a crucial special case, by showing that there is a Levi subgroup of $G$ such that the Harish-Chandra restriction of $\chi$ to $L$ is not irreducible.

Proposition 6.16. Assume $G=H$. Let $I_{\ell}=J \cup K$ be the union of two subsets $J, K$. Let $\chi$ be a $S y l_{p}$-vanishing character of $G$.

(1) Suppose that $\bar{\chi}_{L_{J}}$ and $\bar{\chi}_{L_{K}}$ are irreducible. Then $\chi=S t$.

(2) Suppose that $\bar{\chi}_{T}$ is irreducible and $\left(\bar{\chi}_{L_{J}}, 1_{L_{J}}\right)=\left(\bar{\chi}_{L_{K}}, 1_{L_{K}}\right)=1$. Then $\left(\chi, 1_{G}\right)=1$.

Proof. (1) As $\bar{\chi}_{L_{J}}$ and $\bar{\chi}_{L_{K}}$ are irreducible and $p$-vanishing, they are of defect 0 . By Lemma 6.4 $(2), \bar{\chi}_{L_{J}}(1)=\left|L_{J}\right|_{p}$, and hence $\chi(1)=|G|_{p}$ by Lemma 2.5. Now, $\bar{\chi}_{T}=$ $1_{T}$ by Lemma 6.9 and $\bar{\chi}_{L_{J}}=S t_{L_{J}}$ by Lemma 6.5. Similarly, $\bar{\chi}_{L_{K}}=S t_{L_{K}}$. The equality $\bar{\chi}_{T}=1_{T}$ also means that $\left(1_{B}^{G}, \chi\right)=1$. Let $\tau \in \operatorname{Irr} G$ occur both in $\chi$ and $1_{B}^{G}\left(\right.$ so $\left.\left(\tau, 1_{B}^{G}\right)=1\right)$. By Harish-Chandra reciprocity, $\left(\bar{\chi}_{L_{J}}, S t_{L_{J}}\right)=\left(\chi, S t_{L_{J}}^{\# G}\right)=1$ and $\left(\bar{\chi}_{L_{K}}, S t_{L_{K}}\right)=\left(\chi, S t_{L_{K}}^{\# G}\right)=1$. So $\tau$ occurs with multiplicity 1 in $1_{B}^{G}, S t_{L_{J}}^{\# G}$ and $S t_{L_{K}}^{\# G}$. Let $\tau$ correspond to the linear character $\lambda \in \operatorname{Irr} W$ according to Lemma 6.13. By Lemma 6.13 $(3),\left(\tau, S t_{L_{J}}^{\# G}\right)=\left(\left.\lambda\right|_{W_{J}}, \varepsilon_{W_{J}}\right)$. Similarly, $\left(\tau, S t_{L_{K}}^{\# G}\right)=\left(\left.\lambda\right|_{W_{K}}, \varepsilon_{W_{K}}\right)$. This is only possible if $\lambda=\varepsilon$, and hence $\tau=S t$.

(2) Since $\bar{\chi}_{T}$ is irreducible and $\left(\bar{\chi}_{L_{J}}, 1_{L_{J}}\right)=1$, we have $\bar{\chi}_{T}=1_{T}$. We proceed as in item (1). Let $\tau \in \operatorname{Irr} G$ occur both in $\chi$ and $1_{B}^{G}$ (so $\tau$ corresponds to the linear character $\lambda \in \operatorname{Irr} W$ according to Lemma 6.13). By Lemma 6.13 $(2), 1=\left(\tau, 1_{P_{J}}^{G}\right)=$ $\left(\left.\lambda\right|_{W_{J}}, 1_{W_{J}}\right)$. Similarly, $\left(\left.\lambda\right|_{W_{K}}, 1_{W_{K}}\right)=1$. This is only possible if $\lambda=1_{W}$, and hence $\left(\chi, 1_{G}\right)=1$.

\section{Chevalley groups of BN-Pair Rank 2}

In this section, we deal with Chevalley groups of BN-pair rank 2. These are $S L(3, q), S p(4, q), S U(4, q) S U(5, q), G_{2}(q),{ }^{3} D_{4}(q)$ and ${ }^{2} F_{4}\left(q^{2}\right)$.

In view of Proposition 6.10 we state the following:

Lemma 7.1. Let $G$ be of $B N$-pair rank 2 and let $\nu$ be a multiplicity 1 irreducible constituent of $1_{B}^{G}$. Then, either $\nu=1_{G}$, or $\nu=S t$ or of degree written below:

$G={ }^{2} F_{4}\left(q^{2}\right): \frac{q^{2}\left(q^{6}+1\right)\left(q^{12}+1\right)}{\left(q^{4}+1\right)\left(q^{2}+1\right)}$ and $\frac{q^{10}\left(q^{6}+1\right)\left(q^{12}+1\right)}{\left(q^{4}+1\right)\left(q^{2}+1\right)}$;

$G={ }^{3} D_{4}(q): q^{7}\left(q^{4}-q^{2}+1\right)$ and $q\left(q^{4}-q^{2}+1\right)$;

$G=G_{2}(q): 2$ characters of degree $q\left(q^{4}+q^{2}+1\right) / 3$;

$G=S p(4, q): 2$ characters of degree $q\left(q^{2}+1\right) / 2 ; G=S U(4, q): q\left(q^{2}-q+1\right)$ and $q^{3}\left(q^{2}-q+1\right) ; G=S U(5, q): q^{2}\left(q^{5}+1\right)(q+1)$ and $q^{4}\left(q^{5}+1\right)(q+1)$. 
Proof. These degrees are given in [4, pp. $114-115]$.

7.1. Groups $S L(3, q)$ and $G L(3, q)$. Note that $S L(3, q)$ is the only quasi-simple group of rank 2 where we can avoid substantial computations.

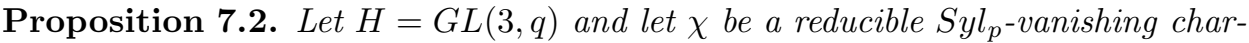
acter of $H$ of degree $q^{3}$. Then $\chi=\mu+\sigma^{\# H}$, where $\mu$ is a linear character of $H$ and $\sigma$ is a cuspidal irreducible character of a proper Levi subgroup $L \neq T$ (and hence $\sigma(1)=q-1)$. In addition, $\sigma^{\# H}$ is irreducible.

Let $\sigma$ be a cuspidal character of $L$. Then the character $1_{H}+\sigma^{\# H}$ is p-vanishing if and only if $Z(L)$ is the kernel of $\sigma$.

Proof. Let $L$ be a Levi subgroup of a parabolic subgroup $P$ of $H$, where $P$ is not a Borel subgroup. Then $L=X Z(L)$, where $X \cong G L(2, q)$.

By Proposition 6.16, $\bar{\chi}_{L}=\left.\mu\right|_{L}+\sigma$, where $\mu(1)=1$ and $\sigma$ is cuspidal. (Indeed, as $L / Z(L) \cong P G L(2, q)$, it follows that $L$ has no irreducible character of degree $d$ with $1<d \leq(q+1) / 2$ for $q \neq 3$. In the latter case $(q-1) / 2$ equals 1 .) By HarishChandra reciprocity, $\left(\chi, \sigma^{\# H}\right)=1$. In addition, as $C_{W}(\sigma)=1$, the Harish-Chandra series of $\sigma$ consists of a single character, see [6, p. 678]. (Note that in our case the ramification group defined in [6, p. 678] is trivial as $N_{H}(L)=L$.) Therefore, $\sigma^{\# H}$ is irreducible, and $\sigma^{\# H}(1)=q^{3}-1$. As $\chi(1)=q^{3}$, the first statement of the lemma follows.

Let $\sigma$ be any cuspidal character of $L$. It is easy to compute that $\sigma^{\# H}(u)=-1$ for every unipotent element $u \neq 1$. So $\mu+\sigma^{\# H}$ is $S y l_{p}$-vanishing for every linear character $\mu$ of $H$. Suppose that $\chi=1_{H}+\sigma^{\# H}$ is $p$-vanishing. Then, by Corollary 6.12, $\sigma$ is trivial on $Z(L)$. Conversely, let $g=s u \in H$, where $s \neq 1$ is semisimple, $u$ is unipotent and $s u=u s$. Then $g$ is conjugate to an element of $L$, and if $g \in L$ then $s \in Z(L)$. Therefore, $\sigma(s u)=\sigma(u)=-1$. Let $C$ be the conjugacy class of $s u$ in $H$. One observes that $C \cap P$ forms a conjugacy class in $P$. Let $\tau$ be the inflation of $\sigma$ to $P$. Then $\tau^{H}=\sigma^{\# H}$ and $\tau(s u)=-1$. By the induced character formula, we have $\tau^{H}(s u)=-1$, which proves the second statement.

Corollary 7.3. Let $G=S L(3, q)$, and let $\chi$ be a p-vanishing character of degree $q^{3}$. Then either $\chi=S t$ or $\left(\chi, 1_{G}\right)=1$. In addition, no irreducible constituent of $\chi$ is cuspidal.

Proposition 7.2 can be compared with the results obtained with use of CHEvIE. Below are some details.

Let $H=G L(3, q)$. Following CHEvie, we partition $\operatorname{Irr} H$ into 8 sets $\mathbf{X}_{h}, 1 \leq$ $h \leq 8$, see Table 11.5.1-A. Applying procedure 4.5 we obtain in step (1) the $S y l_{p^{-}}$ decompositions of Table11.5,1-B and $\Delta_{H}=\{1,2,7,8\}$. In step (2) we get a unique character $\psi_{1}=\chi_{1}+\chi_{7}$. Now, in step (3) we obtain all $S y l_{p}$-vanishing characters of $H$ of degree $|H|_{p}$ (see Table11.51-C). In particular, every such character has a one-dimensional irreducible constituent.

Lemma 7.4. Let $H=G L(3, q)$. Then $H$ admits exactly $(q-1)(q-\delta) / 2$ reducible p-vanishing characters $\psi$ of degree $|H|_{p}$, all of type

$$
\psi=\chi_{1}\left(n_{1}\right) \cdot\left(1_{H}+\chi_{7}\left(q-1, n_{7}\right)\right),
$$

where $\delta=\frac{1-(-1)^{q}}{2}, n_{1} \in \mathbb{Z}_{q-1}$ and $n_{7}=(q-1) n_{7}^{\prime}$, with $n_{7}^{\prime} \in \mathbb{Z}_{q+1}$ and $q+1$ does not divide $n_{7}^{\prime}$. 
Proof. Modulo linear characters of $H$, take $\psi_{1}=1_{H}+\chi_{7}(m, n)$. Then, $(q-1) \mid$ $(m+n)$ and it suffices to look at the classes $C_{5}(a, b)$, where we have $\zeta_{1}^{m(b-a)}=1$. Thus, $m=q-1$ and $n=(q-1) n^{\prime}$, where $n^{\prime} \in \mathbb{Z}_{q+1}$ and $q+1$ does not divide $n^{\prime}$.

Now, let $G=S L(3, q)$. We have to distinguish two cases, depending on whether 3 divides $q-1$ or not. However, the computations remain very similar to those for $G L(3, q)$. We provide the relevant informations in Tables 11.5, 2-A,B,C if $3 \mid(q-1)$, and in Tables 11.5. 3-A,B,C otherwise. Thus, it can be easily proved the following

Lemma 7.5. Let $G=S L(3, q)$ and $\delta=\frac{1-(-1)^{q}}{2}$. Then, $G$ admits exactly $(q-\delta) / 2$ reducible p-vanishing characters $\psi$ of degree $|G|_{p}$, all of type

$$
\psi=1_{G}+\chi_{i}(a)
$$

where $a=(q+1) a^{\prime}, i=10$ if $3 \mid(q-1)$, and $i=7$, otherwise.

7.2. Groups $U(4, q)$ and $U(5, q)$. First, apply Procedure 4.5 to the group $H=$ $U(4, q)$. In step (1) we obtain the $S y l_{p}$-decompositions of Table 11.6-B and $\Delta_{H}=$ $\{2,5,7,8,12,19,23\}$. Note that $\chi_{19}$ and $\chi_{23}$ are the only regular characters of this set. In step (2), let $\psi$ be a $S y l_{p}$-vanishing character of $H$ of degree $|H|_{p}$, whose constituents lie in $\Delta_{H}$. Since the degree of $\chi_{23}$ is greater than $|H|_{p}$, it follows that $\chi_{19}$ is a constituent of $\psi$. So the other constituents of $\psi$ are $\left\{\chi_{h} \mid h=2,5,7,8,12\right\}$. Using the classes $C_{3}(q+1)$ and $C_{5}(q+1)$, we observe that $\psi$ must have at least 2 constituents of type $\chi_{7}$ and a constituent of type $\chi_{8}$. Now, the character values at the class $C_{4}(q+1)$ imply that either $\chi_{2}$ is a constituent of $\psi$, or both $\chi_{8}$ and $\chi_{12}$ are other constituents of $\psi$. In the latter case we get a contradiction on the class $C_{3}(q+1)$. Therefore, we get a unique character $\psi_{1}=\chi_{2}+\chi_{7}+\chi_{7}^{\prime}+\chi_{8}+\chi_{19}$, where $\chi_{7}, \chi_{7}^{\prime} \in \mathbf{X}_{7}$ are not necessarily distinct. Finally, in step (3) we determine all $S y l_{p}$-vanishing characters of $H$ of degree $|H|_{p}$ (see Table 11.6 $\mathrm{C}$ ).

Lemma 7.6. Let $H=U(4, q)$. Then neither $H$ nor $G=H^{\prime}$ has a reducible p-vanishing character of degree $|H|_{p}$.

Proof. Suppose the contrary and assume $q \geq 4$ (the cases $q=2,3$ can be solved with Magma). In view of Table 11.6 $\mathrm{C}$, up to linear characters of $H$, we have to consider the following four cases:

(1) $\psi_{1}=\chi_{2}(0)+\chi_{7}\left(k_{7,1}, k_{7,2}\right)+\chi_{7}\left(k_{7,1}^{\prime}, k_{7,2}^{\prime}\right)+\chi_{8}\left(k_{8,1}, k_{8,2}\right)+\chi_{19}\left(k_{19,1}, k_{19,2}\right.$, $\left.k_{19,3}, k_{19,4}\right)$. On the class $C_{18}(1,2)$ we obtain $\xi_{1}^{k_{8,1}-k_{8,2}}=1$. This implies that $q+1$ divides $k_{8,1}-k_{8,2}$, a contradiction.

(2) $\psi_{2}=\chi_{2}(0)+\chi_{7}\left(k_{7,1}, k_{7,2}\right)+\chi_{16}\left(k_{16,1}, k_{16,2}, k_{16,3}\right)+\chi_{19}\left(k_{19,1}, k_{19,2}, k_{19,3}, k_{19,4}\right)$. On the classes $C_{16}\left(q+1, i_{2},-i_{2}\right)$ we have

$$
\begin{aligned}
2+\operatorname{Re}\left(\xi_{1}^{\left(k_{7,1}-k_{7,2}\right) i_{2}}\right)+ & \operatorname{Re}\left(\xi_{1}^{\left(k_{16,1}-k_{16,2}\right) i_{2}}\right)+\operatorname{Re}\left(\xi_{1}^{\left(k_{16,1}-k_{16,3}\right) i_{2}}\right)+ \\
+\operatorname{Re}\left(\xi_{1}^{\left(k_{16,2}-k_{16,3}\right) i_{2}}\right)= & \operatorname{Re}\left(\xi_{1}^{\left(k_{19,1}-k_{19,2}\right) i_{2}}\right)+\operatorname{Re}\left(\xi_{1}^{\left(k_{19,1}-k_{19,3}\right) i_{2}}\right)+ \\
+\operatorname{Re}\left(\xi_{1}^{\left(k_{19,1}-k_{19,4}\right) i_{2}}\right)+ & +\operatorname{Re}\left(\xi_{1}^{\left(k_{19,2}-k_{19,3}\right) i_{2}}\right)+\operatorname{Re}\left(\xi_{1}^{\left(k_{19,2}-k_{19,4}\right) i_{2}}\right)+ \\
& +\operatorname{Re}\left(\xi_{1}^{\left(k_{19,3}-k_{19,4}\right) i_{2}}\right) .
\end{aligned}
$$

This holds for every $i_{2} \in \mathbb{Z}_{q+1}$ such that $q+1$ does not divide $2 i_{1}$. If $q \geq 13$, this implies that $q+1$ divides $k_{19, i}-k_{19, j}$ for some $i \neq j$, a contradiction. For $4 \leq q \leq 11$, it can be proved directly that this condition for $i_{1}=1, \ldots,\lfloor(q-1) / 2\rfloor$ is never satisfied. 
(3) $\psi_{3}=\chi_{2}(0)+\chi_{7}\left(k_{7,1}, k_{7,2}\right)+\chi_{15}\left(k_{15,1}, k_{15,2}, k_{15,3}\right)$. On the class $C_{8}(1, q-$ 2) we have $\xi_{1}^{k_{7,1}-k_{7,2}}=\xi_{1}^{2 k_{15,1}-k_{15,2}-k_{15,3}}$ and on $C_{18}(1,2)$ we have $1=$ $\xi_{1}^{2 k_{15,1}-k_{15,2}-k_{15,3}}$. So $\xi_{1}^{k_{7,1}-k_{7,2}}=1$, and hence $(q+1) \mid\left(k_{7,1}-k_{7,2}\right)$, a contradiction.

(4) $\psi_{4}=\chi_{2}(0)+\chi_{6}\left(k_{1}, k_{2}\right)$. Using the class $C_{7}(1, q-2)$ we have $\xi_{1}^{k_{2}-k_{1}}=1$. It follows that $(q+1) \mid\left(k_{2}-k_{1}\right)$, a contradiction with the conditions on the parameters.

Since all the conjugacy classes we have considered belong to $H^{\prime}$, it follows from Proposition 6.2 that also $H^{\prime}$ has no $p$-vanishing character of degree $|H|_{p}$.

Next, apply Procedure 4.5 to $H=U(5, q)$. In step (1) we obtain the $S y l_{p^{-}}$ decompositions of Table 11.7. B and $\Delta_{H}=\{2,5,6,7,16,20,21,37,42,43\}$. Note that $\chi_{37}, \chi_{42}$ and $\chi_{43}$ are the only regular characters in this set. In step (2), let $\psi$ be a $S y l_{p}$-vanishing character of $H$ of degree $|H|_{p}$, whose constituents lie in $\Delta_{H}$. Observe that $\chi_{43}$ is ruled out by degree reasons.

(1) Suppose that $\chi_{37}$ is a constituent of $\psi$. Then, looking at the classes $C_{3}(q+1)$ and $C_{7}(q+1)$, the character $\psi$ must have at least one constituent of type $\chi_{21}$ and three constituents of type $\chi_{20}$. With some little efforts, we get a unique $S y l_{p}$-vanishing character $\psi_{1}=\chi_{2}+\chi_{5}+\chi_{16}+\chi_{20}+\chi_{20}^{\prime}+\chi_{20}^{\prime \prime}+\chi_{21}+\chi_{37}$, where $\chi_{20}, \chi_{20}^{\prime}, \chi_{20}^{\prime \prime} \in \mathbf{X}_{20}$ are not necessarily distinct.

(2) Let $\chi_{42}$ be a constituent of $\psi$. Then, by degree reasons, the other constituents of $\psi$ must belong to the set $\left\{\chi_{h} \mid h=5,6,7,16,21\right\}$. Then, looking at the classes $C_{5}(q+1)$ and $C_{7}(q+1)$, the character $\psi$ must have one constituent of type $\chi_{7}$ and at least one constituent of type $\chi_{16}$. This leads to a contradiction on the class $C_{6}(q+1)$.

With step (3) we get all $S y l_{p}$-vanishing characters of $H$ of degree $|H|_{p}$ (see Table 11.7. C).

Lemma 7.7. Let $H=U(5, q)$. Then, neither $H$ nor $G=H^{\prime}$ admits any reducible p-vanishing character of degree $|H|_{p}$.

Proof. Suppose the contrary. The cases $q=2,3$ can be solved using MAgma. Assume $q>3$. First, take $\psi_{14}=\chi_{2}\left(k_{2,1}\right)+\chi_{8}\left(k_{8,1}, k_{8,2}\right)$. Using the class $C_{9}(1, q-3)$ we have $\xi_{1}^{k_{8,2}-k_{8,1}}=1$, a contradiction. In all the other cases, it suffices to consider the classes $C_{34}\left(q+1, i_{2}, i_{2}\right)$. We obtain one of the two following conditions:

(a) $1+\xi_{1}^{\left(k_{a, 2}-k_{a, 1}\right) i_{2}}=\xi_{1}^{\left(k_{b, 1}-k_{b, 2}\right) i_{2}}+\xi_{1}^{\left(k_{b, 1}-k_{b, 3}\right) i_{2}}, i_{2}=1,2$;

(b) $2+\xi_{1}^{\left(k_{a, 2}-k_{a, 1}\right) i_{2}}=\xi_{1}^{\left(k_{b, 1}-k_{b, 2}\right) i_{2}}+\xi_{1}^{\left(k_{b, 1}-k_{b, 3}\right) i_{2}}+\xi_{1}^{\left(k_{b, 1}-k_{b, 4}\right) i_{2}}, i_{2}=1,2,3$.

In both the cases, this implies that $q+1$ divides $k_{b, 1}-k_{b, j}$ for some $j \neq 1$, a contradiction with the conditions on the parameters. (The case (b) for $q=4$ needs to be done directly.)

Since all the conjugacy classes used above belong to $H^{\prime}=G$, if follows from Proposition 6.2 that also $G$ has no $p$-vanishing characters of degree $|G|_{p}$.

7.3. Groups $G=S p(4, q)$. Let $G=S p(4, q)$ with $q$ even and apply Procedure 4.5. In step (1) we get the $S y l_{p}$-decompositions of Table 11.8.1-B and $\Delta_{G}=$ $\{1,2,3,4,5,9,13,18,19\}$. Note that the regular characters belonging to this set are $\chi_{18}$ and $\chi_{19}$. In step (2), let $\psi$ be a $S y l_{p}$-vanishing character of $G$ of degree $|G|_{p}$, whose constituents lie in $\Delta_{G}$. 
(1) Let $\chi_{18}$ be a constituent of $\psi$. Then, by degree reasons, the other constituents of $\psi$ must belong to the set $\left\{\chi_{h} \mid h=1,5\right\}$. In this case we easily obtain a contradiction.

(2) Let $\chi_{19}$ be a constituent of $\psi$. Then, by degree reasons, the other constituents of $\psi$ must belong to the set $\left\{\chi_{h} \mid h=1,2,3,4,5,9,13\right\}$. Looking at the class $C_{5}$, either $\chi_{9}$ or $\chi_{13}$ is a constituent of $\psi$. In the former case, we obtain the $S y l_{p}$-vanishing characters $\psi_{1}=\chi_{1}+\chi_{9}+\chi_{13}+\chi_{19}$ and $\psi_{2}=\chi_{3}+\chi_{5}+\chi_{9}+\chi_{19}$. In the latter case, we obtain the characters $\psi_{1}$ and $\psi_{3}=\chi_{4}+\chi_{5}+\chi_{13}+\chi_{19}$.

With step (3) we are able to list all the $S y l_{p}$-vanishing characters of $G$ of degree $|G|_{p}$ (see Table 11.8.C).

Lemma 7.8. Let $G=S p(4, q), q$ even. Then $G$ has no reducible p-vanishing character of degree $|G|_{p}$.

Proof. Suppose the contrary. Then we have to consider the following cases.

(1) $\psi_{1}=1_{G}+\chi_{9}\left(k_{9}\right)+\chi_{13}\left(k_{13}\right)+\chi_{19}\left(k_{19}, l_{19}\right)$. For $i=1,2$, on the classes $C_{10}(i)$ we have $\operatorname{Re}\left(\xi_{1}^{i k_{19}}\right)+\operatorname{Re}\left(\xi_{1}^{i l_{19}}\right)=\operatorname{Re}\left(\xi_{1}^{i k_{13}}\right)+\operatorname{Re}\left(\xi_{1}^{i k_{9}}\right)$ and on the classes $C_{14}(i)$ we have $\operatorname{Re}\left(\xi_{1}^{\left(k_{19}+l_{19}\right) i}\right)+\operatorname{Re}\left(\xi_{1}^{\left(k_{19}-l_{19}\right) i}\right)=\operatorname{Re}\left(\xi_{1}^{2 i k_{13}}\right)+\operatorname{Re}\left(\xi_{1}^{i k_{9}}\right)$. Set $A=\operatorname{Re}\left(\xi_{1}^{k_{19}}\right), B=\operatorname{Re}\left(\xi_{1}^{l_{19}}\right), C=\operatorname{Re}\left(\xi_{1}^{k_{13}}\right)$ and $D=\operatorname{Re}\left(\xi_{1}^{k_{9}}\right)$ :

$$
\left\{\begin{array}{l}
2 A B=2 C^{2}-1+D \\
(2 A B)^{2}-2\left(A^{2}+B^{2}\right)+1=4 C^{4}-4 C^{2}+D^{2} \\
(A, B)=(C, D),(D, C)
\end{array} .\right.
$$

If $C=\frac{1}{2}$, then $2 A B=D-\frac{1}{2}$. This implies that either $A=C=\frac{1}{2}$ and $B=D=D-1 / 2$ or $B=C=\frac{1}{2}$ and $A=D=D-\frac{1}{2}$. So, in both the cases we have an absurd. Hence, we may assume $C \neq \frac{1}{2}$ and $D=\frac{2 C^{2}-1}{2 C-1}$. We get the equation

$$
8(C-1)^{2}\left(C^{3}+\frac{1}{2} C^{2}-\frac{1}{2} C-\frac{1}{8}\right)=0 .
$$

Now, since $C \neq 1$ :

(7.1) $0=C^{3}+\frac{1}{2} C^{2}-\frac{1}{2} C-\frac{1}{8}=\left(C-\cos \left(\frac{2 \pi}{7}\right)\right)\left(C-\cos \left(\frac{4 \pi}{7}\right)\right)\left(C-\cos \left(\frac{6 \pi}{7}\right)\right)$.

Thus, we have a solution if and only if 7 divides $q+1$. However, this does not happen since $q$ is even.

(2) $\psi_{2}=1_{G}+\chi_{9}\left(k_{9}\right)+\chi_{14}\left(k_{14}\right)$. On the class $C_{14}(1)$ we have $\operatorname{Re}\left(\xi_{1}^{k_{9}}\right)=1$, a contradiction.

(3) $\psi_{3}=\chi_{1}+\chi_{10}\left(k_{10}\right)+\chi_{13}\left(k_{13}\right)$. On the class $C_{10}(1)$ we have $\operatorname{Re}\left(\xi_{1}^{k_{13}}\right)=1$, a contradiction.

(4) Take $\psi_{4}=\chi_{3}+\chi_{5}+\chi_{9}\left(k_{9}\right)+\chi_{19}\left(k_{19}, l_{19}\right)$. On the classes $C_{14}(i)(i=1,2)$ we have $1+\operatorname{Re}\left(\xi_{1}^{2 i k_{9}}\right)=\operatorname{Re}\left(\xi_{1}^{i\left(k_{19}+l_{19}\right)}\right)+\operatorname{Re}\left(\xi_{1}^{i\left(k_{19}-l_{19}\right)}\right)$, which implies that $q+1$ divides either $k_{19}+l_{19}$ or $k_{19}-l_{19}$. In both cases, we get a contradiction

(5) $\psi_{5}=\chi_{3}+\chi_{5}+\chi_{10}(k)$. On the class $C_{14}(1)$ we have $R e\left(\xi_{1}^{k}\right)=1$, a contradiction.

(6) $\psi_{6}=\chi_{4}+\chi_{5}+\chi_{13}\left(k_{13}\right)+\chi_{19}\left(k_{19}, l_{19}\right)$. On the classes $C_{14}(i)(i=1,2)$ we have $1+\operatorname{Re}\left(\xi_{1}^{2 i k_{13}}\right)=\operatorname{Re}\left(\xi_{1}^{i\left(k_{19}+l_{19}\right)}\right)+\operatorname{Re}\left(\xi_{1}^{i\left(k_{19}-l_{19}\right)}\right)$, which implies 
that $q+1$ divides either $k_{19}+l_{19}$ or $k_{19}-l_{19}$. In both cases, we get a contradiction.

(7) $\psi_{7}=\chi_{4}+\chi_{5}+\chi_{14}(k)$. On the class $C_{10}(1)$ we have $\operatorname{Re}\left(\xi_{1}^{k}\right)=1$, a contradiction.

Let $G=S p(4, q), q$ odd. The character table of this group is not implemented in CHEvie, but it was described in papers by Srinivasan [21, Przygocki [19] and Bleher 11. We keep the notation of 21 for the conjugacy classes. We partition $\operatorname{Irr} G$ into 49 sets $\mathbf{X}_{h}$ as done in Table 11.8,2-A. (Note that this is not taken from CHEVIE but again all characters in every set are $S y l_{p}$-equivalent.)

Applying Procedure 4.5 we obtain in step (1) the $S y l_{p}$-decompositions of Table 11.8, 2-B and $\Delta_{G}=\{6,16,17,37,38,40,42,44\}$. When we consider step (2), we note that the only regular characters in $\Delta_{G}$ are $\chi_{16}$ and $\chi_{17}$. By Lemma 3.1 at least one of them is a constituent of a $S y l_{p}$-vanishing character $\psi$ of degree $|G|_{p}$ whose constituents lie in $\Delta_{G}$. Inspecting [20, we observe that $\chi_{16}$ and $\chi_{17}$ are $C S p(4, q)$ conjugate. By Proposition 6.2, both of them are constituents of $\psi$. (It follows that they are constituents of two distinct non-degenerate Gelfand-Graev characters.) Working only with these constituents, we obtain only two $S y l_{p}$-vanishing characters of degree $|G|_{p}$ :

$$
\psi_{1}=\chi_{6}+\chi_{16}+\chi_{17}+\chi_{37}+\chi_{38}+\chi_{40}+\chi_{40}^{\prime}+\chi_{44}
$$

and

$$
\psi_{2}=\chi_{16}+\chi_{17}+\chi_{37}+\chi_{38}+\chi_{40}+\chi_{40}^{\prime}+\chi_{40}^{\prime \prime}+\chi_{42} .
$$

Now step (3) produces all $S y l_{p}$-vanishing characters of $G$ of degree $|G|_{p}$ (see Table 11.8, 2-C).

Lemma 7.9. Let $G=S p(4, q), q$ odd. Then $G$ admits reducible $p$-vanishing characters $\psi$ of degree $|G|_{p}$ if and only if $7 \mid(q+1)$. In this case, there are exactly 3 characters $\psi$, all of type

$$
\psi=1_{G}+\chi_{4}(a, b)+\chi_{6}(c)+\chi_{10}(d),
$$

where

$$
(a, b, c, d) \in\{(T, 3 T, T, 3 T),(T, 2 T, 2 T, T),(2 T, 3 T, 3 T, 2 T)\}
$$

and $T=(q+1) / 7$.

Proof. Most of the $S y l_{p}$-vanishing characters of $G$ can be easily ruled out. We present here some examples for the most interesting cases.

(1) Let $\psi_{1}=1_{G}+\chi_{4}\left(k_{4}, l_{4}\right)+\chi_{6}\left(k_{6}\right)+\chi_{10}\left(k_{10}\right)$. On the class $D_{31}$ we have $(-1)^{k_{4}}+(-1)^{l_{4}}=(-1)^{k_{6}}+(-1)^{k_{10}}$ On the class $D_{21}$ we have $(q-1)(-1)^{k_{6}}+$ $q=(q-1)\left((-1)^{k_{4}}+(-1)^{l_{4}}\right)+(-1)^{k_{10}}$. Thus, $k_{10}$ is even. On the class $B_{7}(i)$ we have $\operatorname{Re}\left(\xi_{1}^{\left(k_{4}+l_{4}\right) i}\right)+\operatorname{Re}\left(\xi_{1}^{\left(k_{4}-l_{4}\right) i}\right)=\operatorname{Re}\left(\xi_{1}^{2 i k_{6}}\right)+\operatorname{Re}\left(\xi_{1}^{i k_{10}}\right)$ and on the class $C_{21}(i): \operatorname{Re}\left(\xi_{1}^{k_{4} i}\right)+\operatorname{Re}\left(\xi_{1}^{l_{4} i}\right)=\operatorname{Re}\left(\xi_{1}^{i k_{6}}\right)+\operatorname{Re}\left(\xi_{1}^{i k_{10}}\right)$.

We set $A=\operatorname{Re}\left(\xi_{1}^{k_{4}}\right), B=\operatorname{Re}\left(\xi_{1}^{l_{4}}\right), C=\operatorname{Re}\left(\xi_{1}^{k_{6}}\right)$ and $D=\operatorname{Re}\left(\xi_{1}^{k_{10}}\right)$, obtaining

$$
\left\{\begin{array}{l}
2 A B=2 C^{2}-1+D \\
(2 A B)^{2}-2\left(A^{2}+B^{2}\right)+1=4 C^{4}-4 C^{2}+D^{2} \\
(A, B)=(C, D),(D, C)
\end{array} .\right.
$$


If $C=\frac{1}{2}$, then $2 A B=D-\frac{1}{2}$. This implies that either $A=C=\frac{1}{2}$ and $B=D=D-1 / 2$ or $B=C=\frac{1}{2}$ and $A=D=D-\frac{1}{2}$. So, in both the cases we have an absurd. Hence, we may assume $C \neq \frac{1}{2}$ and $D=\frac{2 C^{2}-1}{2 C-1}$. We get the equation

$$
8(C-1)^{2}\left(C^{3}+\frac{1}{2} C^{2}-\frac{1}{2} C-\frac{1}{8}\right)=0,
$$

and so Equation (7.1) which implies $C=\cos (2 t \pi / 7), t=1,2,3$, and $D=$ $\cos (6 t \pi / 7)$. Thus,

$$
\begin{aligned}
(A, B, C, D) \in \quad & \left(\cos \frac{2 \pi}{7}, \cos \frac{6 \pi}{7}, \cos \frac{2 \pi}{7}, \cos \frac{6 \pi}{7}\right), \\
& \left(\cos \frac{6 \pi}{7}, \cos \frac{2 \pi}{7}, \cos \frac{2 \pi}{7}, \cos \frac{6 \pi}{7}\right), \\
& \left(\cos \frac{4 \pi}{7}, \cos \frac{2 \pi}{7}, \cos \frac{4 \pi}{7}, \cos \frac{2 \pi}{7}\right) \\
& \left(\cos \frac{2 \pi}{7}, \cos \frac{4 \pi}{7}, \cos \frac{4 \pi}{7}, \cos \frac{2 \pi}{7}\right) \\
& \left(\cos \frac{6 \pi}{7}, \cos \frac{4 \pi}{7}, \cos \frac{6 \pi}{7}, \cos \frac{4 \pi}{7}\right), \\
& \left.\left(\cos \frac{4 \pi}{7}, \cos \frac{6 \pi}{7}, \cos \frac{6 \pi}{7}, \cos \frac{4 \pi}{7}\right)\right\} .
\end{aligned}
$$

This means, denoting $T=\frac{q+1}{7}$, that

$\left(k_{4}, l_{4}, k_{6}, k_{10}\right) \in\{(T, 3 T, T, 3 T),(3 T, T, T, 3 T),(2 T, T, 2 T, T)$

$$
(T, 2 T, 2 T, T),(3 T, 2 T, 3 T, 2 T),(2 T, 3 T, 3 T, 2 T)\} .
$$

It is clear now that $\operatorname{Re}\left(\xi_{1}^{\left(k_{4}+l_{4}\right) i}\right)+\operatorname{Re}\left(\xi_{1}^{\left(k_{4}-l_{4}\right) i}\right)=\operatorname{Re}\left(\xi_{1}^{2 i k_{6}}\right)+\operatorname{Re}\left(\xi_{1}^{i k_{10}}\right)$ and $\operatorname{Re}\left(\xi_{1}^{i k_{4}}\right)+\operatorname{Re}\left(\xi_{1}^{i l_{4}}\right)=\operatorname{Re}\left(\xi_{1}^{i k_{6}}\right)+\operatorname{Re}\left(\xi_{1}^{i k_{10}}\right)$ for all $i$. It follows that $\psi$ is a $p$-vanishing character if and only if 7 divides $q+1$. In this case, since $\chi_{4}(a, b)=\chi_{4}(b, a), G$ admits exactly three $p$-vanishing characters of degree $|G|_{p}$ as described in the statement.

(2) Take $\psi_{16}=1_{G}+\chi_{6}\left(k_{6}\right)+\chi_{10}\left(k_{10}\right)+\chi_{16}\left(k_{16}\right)+\chi_{17}\left(k_{17}\right)$. Looking at classes $C_{21}(1)$ and $C_{22}(1)$ we obtain $\operatorname{Re}\left(\xi_{1}^{k_{16}}\right)=\operatorname{Re}\left(\xi_{1}^{k_{17}}\right)$. On the classes $B_{7}(i)(i=1,2)$ we get $\operatorname{Re}\left(\xi_{1}^{2 i k_{6}}\right)+\operatorname{Re}\left(\xi_{1}^{i k_{10}}\right)=2(-1)^{i} \operatorname{Re}\left(\xi_{1}^{i k_{16}}\right)$. Hence $\operatorname{Re}\left(\xi_{1}^{k_{10}}\right)=-1$, a contradiction.

(3) $\psi_{29}=1_{G}+\chi_{6}\left(k_{6}\right)+\chi_{16}\left(k_{16}\right)+\chi_{23}+\chi_{24}$. On the classes $C_{21}(1)$ and $C_{22}(1)$ we obtain $\operatorname{Re}\left(\xi_{1}^{k_{16}}\right)=1$, a contradiction.

Remark 7.10. It can be shown that the $p$-vanishing characters $\psi_{1}$ of $S p(4, q)$, described above, can be extended to $p$-vanishing characters of $C S p(4, q)$. In Tables 11.8. 3-A,B,C we report information about irreducible characters, $S y l_{p}$-decompositions and $S y l_{p}$-vanishing characters of $C S p(4, q), q$ odd.

7.4. Groups ${ }^{3} D_{4}(q), G_{2}(q)$ and ${ }^{2} F_{4}\left(q^{2}\right)$. Let $G={ }^{3} D_{4}(q)$. The character table of $G$ depends on the parity of $q$. Here we describe in detail only the case $q$ odd; the case $q$ even is very similar (see Tables 11.9 1.A,B,C).

So, let $q$ be odd and apply Procedure 4.5 In step (1) we obtain the $S y l_{p^{-}}$ decompositions of Table 11.9 2-B and $\Delta_{G}=\{1,2,3,4,5,6,7,9,15,16,21,24,25,30$, 
$33\}$. Note that, for these $h$, the only regular characters $\chi_{h}$ are with $h=30,33$. In step (2), let $\psi$ be a $S y l_{p}$-vanishing character of $G$ of degree $|G|_{p}$, whose constituents lie in $\Delta_{G}$. First, some computation shows that $\left(\chi_{30}, \psi\right)=0$.

Next, assume that $\chi_{33}$ is a constituent of $\psi$. Looking at the values of $\chi_{h}$ at the class $C_{7}$, we observe that $\chi_{21}$ or $\chi_{24}$ is a constituent of $\psi$. In the former case, we get the character $\psi_{1}=\chi_{1}+\chi_{21}+\chi_{24}+\chi_{25}+\chi_{33}$. In the latter case, we obtain the characters $\psi_{2}=\chi_{5}+\chi_{6}+\chi_{7}+\chi_{21}+\chi_{33}$ and $\psi_{3}=\chi_{2}+\chi_{5}+\chi_{6}+\chi_{24}+\chi_{25}+\chi_{33}$. With step (3) we determine all $S y l_{p}$-vanishing characters of $G$ of degree $|G|_{p}$ (see Table 11.92-C).

Lemma 7.11. The group $G={ }^{3} D_{4}(q)$ has no reducible p-vanishing character of degree $|G|_{p}$.

Proof. Suppose the contrary. Assume that $q$ is odd (the case of $q$ even is similar).

(1) Take $\psi_{1}=1_{G}+\chi_{21}\left(k_{21}\right)+\chi_{24}\left(k_{24}\right)+\chi_{25}\left(k_{25}\right)+\chi_{33}\left(k_{33}, l_{33}\right)$. On the classes $C_{22}(a)(a=1,2,3)$ we have

$$
1+\operatorname{Re}\left(\xi_{1}^{a k_{21}}\right)+\operatorname{Re}\left(\xi_{1}^{2 a k_{21}}\right)=\operatorname{Re}\left(\xi_{1}^{a l_{33}}\right)+\operatorname{Re}\left(\xi_{1}^{a\left(k_{33}+l_{33}\right)}\right)+\operatorname{Re}\left(\xi_{1}^{a\left(k_{33}+2 l_{33}\right)}\right) .
$$

This implies that $q+1$ divides at least one of the numbers $l_{33}, k_{33}+l_{33}$, $k_{33}+2 l_{33}$, which violates the description of the parameter set $I_{h}$.

(2) Take $\psi_{2}=\chi_{5}+\chi_{6}+\chi_{7}+\chi_{21}\left(k_{21}\right)+\chi_{33}\left(k_{33}, l_{33}\right)$. In this case, we have the same contradiction as in the previous case.

(3) Take $\psi_{3}=\chi_{2}+\chi_{5}+\chi_{6}+\chi_{24}\left(k_{24}\right)+\chi_{25}\left(k_{25}\right)+\chi_{33}\left(k_{33}, l_{33}\right)$. On the class $C_{22}(1)$ we obtain $\operatorname{Re}\left(\xi_{1}^{l_{33}}\right)+\operatorname{Re}\left(\xi_{1}^{k_{33}+l_{33}}\right)+\operatorname{Re}\left(\xi_{1}^{k_{33}+2 l_{33}}\right)=3$. This leads to a contradiction.

(4) Take $\psi_{4}=1_{G}+\chi_{21}\left(k_{21}\right)+\chi_{27}\left(k_{27}\right)+\chi_{33}\left(k_{33}, l_{33}\right)$. On the class $C_{22}(a)$ we get

$\operatorname{Re}\left(\xi_{1}^{a k_{21}}\right)+\operatorname{Re}\left(\xi_{1}^{2 a k_{21}}\right)+\operatorname{Re}\left(\xi_{1}^{a k_{27}}\right)=\operatorname{Re}\left(\xi_{1}^{a l_{33}}\right)+\operatorname{Re}\left(\xi_{1}^{a\left(k_{33}+l_{33}\right)}\right)+\operatorname{Re}\left(\xi_{1}^{a\left(k_{33}+2 l_{33}\right)}\right)$.

Using the classes $C_{25}(1)$ and $C_{26}(1)$ we get $\operatorname{Re}\left(\varphi_{6}^{k_{27}}\right)=\operatorname{Re}\left(\varphi_{6}^{k_{33}}\right)=1$. Furthermore, setting $k_{27}=\left(q^{2}-q+1\right) k_{27}^{\prime}$ and $k_{33}=\left(q^{2}-q+1\right) k_{33}^{\prime}$, on the classes $C_{28}(a)$ we have

$\operatorname{Re}\left(\xi_{1}^{a k_{21}}\right)+\operatorname{Re}\left(\xi_{1}^{a k_{27}^{\prime}}\right)+\operatorname{Re}\left(\xi_{1}^{2 a k_{27}^{\prime}}\right)=\operatorname{Re}\left(\xi_{1}^{a k_{33}^{\prime}}\right)+\operatorname{Re}\left(\xi_{1}^{a\left(k_{33}^{\prime}+l_{33}\right)}\right)+\operatorname{Re}\left(\xi_{1}^{a\left(2 k_{33}^{\prime}+l_{33}\right)}\right)$.

With some work, this yields a contradiction.

(5) Take $\psi_{5}=\chi_{1}+\chi_{21}\left(k_{21}\right)+\chi_{28}\left(k_{28}\right)$. On the classes $C_{22}(a)(a=1,2)$ we obtain $1+\operatorname{Re}\left(\xi_{1}^{a k_{28}}\right)=\operatorname{Re}\left(\xi_{1}^{a k_{21}}\right)+\operatorname{Re}\left(\xi_{1}^{2 a k_{21}}\right)$, which implies $(q+1) / 2 \mid k_{21}$, a contradiction.

(6) Take $\psi_{6}=1_{G}+\chi_{22}\left(k_{22}\right)+\chi_{24}\left(k_{24}\right)+\chi_{25}\left(k_{25}\right)$. On the class $C_{22}(1)$ we obtain the equality $\operatorname{Re}\left(\xi_{1}^{k_{22}}\right)=1$, which is a contradiction.

(7) Take $\psi_{7}=1_{G}+\chi_{22}\left(k_{22}\right)+\chi_{27}\left(k_{27}\right)$. On the class $C_{22}(1)$ we obtain $\operatorname{Re}\left(\xi_{1}^{k_{22}}\right)=\operatorname{Re}\left(\xi_{1}^{k_{27}}\right)$ and on the class $C_{26}(1)$ we get $\operatorname{Re}\left(\varphi_{6}^{k_{27}}\right)=1$. On the class $C_{28}(1)$ we have $\operatorname{Re}\left(\xi_{3}^{q(q-1) k_{27}}\right)=1$. This implies that $\left(q^{3}+1\right) / 2$ divides $k_{27}$, which is a contradiction.

(8) Take $\psi_{8}=\chi_{2}+\chi_{5}+\chi_{6}+\chi_{27}\left(k_{27}\right)+\chi_{33}\left(k_{33}\right)$. On the classes $C_{22}(a)$ $(a=1,2,3)$ we get

$$
2+\operatorname{Re}\left(\xi_{1}^{a k_{27}}\right)=\operatorname{Re}\left(\xi_{1}^{a l_{33}}\right)+\operatorname{Re}\left(\xi_{1}^{a\left(k_{33}+l_{33}\right)}\right)+\operatorname{Re}\left(\xi_{1}^{a\left(k_{33}+2 l_{33}\right)}\right) .
$$

This implies that $q+1$ divides at least one of the numbers $l_{33}, k_{33}+l_{33}$, $k_{33}+2 l_{33}$, which is a contradiction. 
(9) Take $\psi_{9}=\chi_{2}+\chi_{5}+\chi_{6}+\chi_{28}\left(k_{28}\right)$. On the class $C_{22}(1)$ we have $R e\left(\xi_{1}^{k_{28}}\right)=1$, a contradiction.

(10) Take $\psi_{10}=\chi_{5}+\chi_{6}+\chi_{7}+\chi_{22}\left(k_{22}\right)$. On the class $C_{22}(1)$ we obtain $\operatorname{Re}\left(\xi_{1}^{k_{22}}\right)=1$, a contradiction.

Now, let $G=G_{2}(q)$. The character table of $G$ provided by CHEVIE distinguishes five cases, depending on the parity of $q$ and on the congruence class of $q$ modulo 3 . All the cases are very similar, so we provide details only for the case with $q$ odd, $q \equiv-1(\bmod 3)$, which requires more work.

So, let $q$ be odd with $3 \mid(q+1)$ and apply Procedure 4.5. With step (1) we obtain the $S y l_{p}$-decompositions of Table 11.10. 5 -B and $\Delta_{G}=\{1,2,3,5,6,7,9,11,15,22,27$, $30\}$. Note that $\chi_{27}$ and $\chi_{30}$ are the only regular characters of this set.

In step (2), let $\psi$ be a $S y l_{p}$-vanishing character of $G$ of degree $|G|_{p}$, whose constituents lie in $\Delta_{G}$. First, assume that $\chi_{27}$ is a constituent of $\psi$. Using the class $C_{7}$, we conclude that either $\chi_{15}$ or $\chi_{22}$ occurs as a constituent of $\psi$. In the former case, we get $\psi_{1}=\chi_{5}+\chi_{6}+\chi_{6}+\chi_{7}+\chi_{7}+\chi_{9}+\chi_{15}+\chi_{27}$. In the latter case, we get $\psi_{2}=\chi_{1}+\chi_{6}+\chi_{7}+\chi_{9}+\chi_{15}+\chi_{22}+\chi_{27}$. Next, assume that $\chi_{30}$ is a constituent of $\psi$. Then, with some work we obtain a contradiction.

Finally, in step (3) we determine all $S y l_{p}$-vanishing characters of $G$ of degree $|G|_{p}$ (see Table 11.10,5-C).

Lemma 7.12. The group $G=G_{2}(q)$ has no reducible p-vanishing character of degree $|G|_{p}$.

Proof. Suppose the contrary. As before, we consider here only the case with $q$ odd and $3 \mid(q+1)$. The proof for the other cases is very similar.

(1) Take $\psi_{1}, \psi_{2}, \psi_{3}, \psi_{4}, \psi_{5}, \psi_{13}$. All these characters do not vanish on the class $C_{14}$.

(2) Take $\psi_{6}=1_{G}+\chi_{15}+\chi_{16}+\chi_{22}\left(k_{22}\right)+\chi_{27}\left(k_{27}, l_{27}\right)$. On the classes $C_{23}(i)$ $(i=1,2,3)$, we have

$$
1+\operatorname{Re}\left(\xi_{1}^{i k_{22}}\right)+\operatorname{Re}\left(\xi_{1}^{2 i k_{22}}\right)=\operatorname{Re}\left(\xi_{1}^{i\left(k_{27}+l_{27}\right)}\right)+\operatorname{Re}\left(\xi_{1}^{i\left(k_{27}-2 l_{27}\right)}\right)+\operatorname{Re}\left(\xi_{1}^{i\left(2 k_{27}-l_{27}\right)}\right) .
$$

This implies that $q+1$ divides at least one of the integers $k_{27}+l_{27}, k_{27}-2 l_{27}$, $2 k_{27}-l_{27}$, a contradiction.

(3) Take $\psi_{7}=1_{G}+\chi_{15}+\chi_{16}+\chi_{23}\left(k_{23}\right)$. On the class $C_{23}(1)$ we have $\operatorname{Re}\left(\xi_{1}^{k_{23}}\right)=$ 1 , a contradiction.

(4) Take $\psi_{8}=1_{G}+\chi_{22}\left(k_{22}\right)+\chi_{24}\left(k_{24}\right)+\chi_{27}\left(k_{27}, l_{27}\right)$. On the classes $C_{23}(i)$ we have

$\operatorname{Re}\left(\xi_{1}^{i k_{22}}\right)+\operatorname{Re}\left(\xi_{1}^{2 i k_{22}}\right)+\operatorname{Re}\left(\xi_{1}^{3 i k_{24}}\right)=\operatorname{Re}\left(\xi_{1}^{i\left(k_{27}+l_{27}\right)}\right)+\operatorname{Re}\left(\xi_{1}^{i\left(k_{27}-2 l_{27}\right)}\right)+\operatorname{Re}\left(\xi_{1}^{i\left(2 k_{27}-l_{27}\right)}\right)$ and on the classes $C_{25}(i)$ we have

$$
\operatorname{Re}\left(\xi_{1}^{i k_{22}}\right)+\operatorname{Re}\left(\xi_{1}^{i k_{24}}\right)+\operatorname{Re}\left(\xi_{1}^{2 i k_{24}}\right)=\operatorname{Re}\left(\xi_{1}^{i k_{27}}\right)+\operatorname{Re}\left(\xi_{1}^{i l_{27}}\right)+\operatorname{Re}\left(\xi_{1}^{i\left(k_{27}-l_{27}\right)}\right) .
$$

These conditions lead to a contradiction.

(5) Take $\psi_{9}=1_{G}+\chi_{22}\left(k_{22}\right)+\chi_{25}\left(k_{25}\right)$. On the classes $C_{23}(i)(i=1,2)$ we have $1+\operatorname{Re}\left(\xi_{1}^{3 i k_{25}}\right)=\operatorname{Re}\left(\xi_{1}^{i k_{22}}\right)+\operatorname{Re}\left(\xi_{1}^{2 i k_{22}}\right)$. This implies that $(q+1) / 2$ divides $k_{22}$, which is a contradiction. 
(6) Take $\psi_{10}=1_{G}+\chi_{23}\left(k_{23}\right)+\chi_{24}\left(k_{24}\right)$. On the classes $C_{25}(i)(i=1,2)$ we obtain $1+\operatorname{Re}\left(\xi_{1}^{i k_{23}}\right)=\operatorname{Re}\left(\xi_{1}^{i k_{24}}\right)+\operatorname{Re}\left(\xi_{1}^{2 i k_{24}}\right)$. This implies that $(q+1) / 2$ divides $k_{24}$, a contradiction.

(7) Take $\psi_{11}=\chi_{4}+\chi_{6}+\chi_{7}+\chi_{22}\left(k_{22}\right)+\chi_{27}\left(k_{27}, l_{27}\right)$. On the classes $C_{23}(i)$ $(i=1,2,3)$ we obtain

$1+\operatorname{Re}\left(\xi_{1}^{i k_{22}}\right)+\operatorname{Re}\left(\xi_{1}^{2 i k_{22}}\right)=\operatorname{Re}\left(\xi_{1}^{i\left(k_{27}+l_{27}\right)}\right)+\operatorname{Re}\left(\xi_{1}^{i\left(k_{27}-2 l_{27}\right)}\right)+\operatorname{Re}\left(\xi_{1}^{i\left(2 k_{27}+l_{27}\right)}\right)$.

This implies that $q+1$ divides at least one of the numbers $k_{27}+l_{27}, k_{27}-2 l_{27}$, $2 k_{27}-l_{27}$, which contradicts the description of the parameter sets $I_{h}$.

(8) Take $\psi_{12}=\chi_{4}+\chi_{6}+\chi_{7}+\chi_{23}\left(k_{23}\right)$. On the class $C_{23}(1)$ we obtain $\operatorname{Re}\left(\xi_{1}^{k_{23}}\right)=1$, a contradiction.

(9) Take $\psi_{14}=\chi_{5}+\chi_{6}+\chi_{7}+\chi_{15}+\chi_{16}+\chi_{27}\left(k_{27}, l_{27}\right)$. On the classes $C_{23}(1)$ we obtain $\operatorname{Re}\left(\xi_{1}^{k_{27}+l_{27}}\right)+\operatorname{Re}\left(\xi_{1}^{k_{27}-2 l_{27}}\right)+\operatorname{Re}\left(\xi_{1}^{2 k_{27}+l_{27}}\right)=3$, which implies that $(q+1)$ divides $\left(k_{27}+l_{27}\right)$, a contradiction.

(10) Take $\psi_{15}=\chi_{5}+\chi_{6}+\chi_{7}+\chi_{24}\left(k_{24}\right)+\chi_{27}\left(k_{27}, l_{27}\right)$. On the classes $C_{23}(i)$ $(i=1,2,3)$ we obtain

$\operatorname{Re}\left(\xi_{1}^{i\left(k_{27}+l_{27}\right)}\right)+\operatorname{Re}\left(\xi_{1}^{i\left(k_{27}-2 l_{27}\right)}\right)+\operatorname{Re}\left(\xi_{1}^{i\left(2 k_{27}-l_{27}\right)}\right)=2+\operatorname{Re}\left(\xi_{1}^{3 i k_{24}}\right)$,

which implies that $q+1$ divides at least one of the numbers $k_{27}+l_{27}$, $k_{27}-2 l_{27}, 2 k_{27}-l_{27}$, which is a contradiction.

(11) Take $\psi_{16}=\chi_{5}+\chi_{6}+\chi_{7}+\chi_{25}\left(k_{25}\right)$. On the class $C_{23}(1)$ we have $\operatorname{Re}\left(\xi_{1}^{3 k_{25}}\right)=$ 1 , a contradiction.

Observe that the group $G=G_{2}(2)^{\prime}$ has no reducible $S y l_{2}$-vanishing characters of degree $|G|_{2}$. On the other hand, $G$ has six reducible $S y l_{2}$-vanishing characters of degree $2|G|_{2}$. In the notation of $[3]$ :

(1) $\phi_{1}=2 \chi_{13}$;

(2) $\phi_{2}=2 \chi_{14}$

(3) $\phi_{3}=\chi_{13}+\chi_{14}$;

(4) $\phi_{4}=\chi_{1}+\chi_{3}+\chi_{4}+\chi_{9}+\chi_{11}$

(5) $\phi_{5}=\chi_{1}+\chi_{3}+\chi_{5}+\chi_{8}+\chi_{12}$;

(6) $\phi_{6}=\chi_{1}+\chi_{3}+\chi_{6}+\chi_{8}+\chi_{9}$.

The three characters $\phi_{1}, \phi_{2}, \phi_{3}$ are 2-vanishing, whereas the other three characters are not. This can be easily proved using the character table of $G$.

Finally, let $G={ }^{2} F_{4}\left(q^{2}\right)$, with $q^{2}=2^{2 m+1}$ and $m \geq 0$. The groups ${ }^{2} F_{4}\left(q^{2}\right)$ require a much larger amount of computations. Note that the official distribution of CHEvIE contains only a partial character table of these groups. In this case, our computations are based on the character table kindly provided by F. Himstedt (constructed for [12]). This table is available in CHEviE format and so we may apply the same argument as in the previous cases.

Applying Procedure 4.5, in step (1) we obtain the $S y l_{p}$-decompositions of Table 11.11-B and $\Delta_{G}=\{1,2,3,4,5,6,7,8,9,10,11,12,13,14,15,16,17,18,19,20,29,30$, $38,40,41,42,43,44,46,47,48,51\}$. Considering step (2), let $\psi$ be a $S y l_{p}$-vanishing character of degree $|G|_{p}$ whose constituents lie in $\Delta_{G}$. If $\left(\chi_{51}, \psi\right)>0$, we obtain 
two characters $\psi$ :

$$
\begin{aligned}
& \psi_{1}=\chi_{2}+\chi_{4}+\chi_{10}+\chi_{11}+\chi_{13}+\chi_{17}+\chi_{20}+\chi_{46}+\chi_{47}+\chi_{47}+\chi_{48}+\chi_{51} \\
& \psi_{2}=\chi_{3}+\chi_{4}+\chi_{10}+\chi_{12}+\chi_{14}+\chi_{17}+\chi_{19}+\chi_{46}+\chi_{47}+\chi_{48}+\chi_{48}+\chi_{51}
\end{aligned}
$$

On the other side, if $\chi_{38}, \chi_{40}$ or $\chi_{41}$ is a constituent of $\psi$, we get a contradiction. With step (3) we determine all $S y l_{p}$-vanishing characters of $G$ of degree $|G|_{p}$ (see Table 11.11.C).

Lemma 7.13. The group $G={ }^{2} F_{4}\left(q^{2}\right)$ has no reducible p-vanishing character of degree $|G|_{p}$.

Proof. Suppose the contrary. According with Table 11.11 C, there are four cases.

(1) Take $\psi_{1}=\chi_{2}+\chi_{4}+\chi_{10}+\chi_{11}+\chi_{13}+\chi_{17}+\chi_{20}+\chi_{46}\left(k_{46}\right)+\chi_{47}\left(k_{47}\right)$ $+\chi_{47}\left(k_{47}^{\prime}\right)+\chi_{48}\left(k_{48}\right)+\chi_{51}\left(k_{51}, l_{51}\right)$. Comparing the values at the classes $C_{42}(a)$ and $C_{43}(a)(a=1, \ldots, 4)$ we obtain

$\operatorname{Re}\left(\left(\varphi_{8}^{\prime}\right)^{\left(a q k_{47} \sqrt{2}\right) / 2}\right)+\operatorname{Re}\left(\left(\varphi_{8}^{\prime}\right)^{\left(a k_{47}(q \sqrt{2}+2) / 2\right)}\right)+\operatorname{Re}\left(\left(\varphi_{8}^{\prime}\right)^{\left(a q k_{47}^{\prime} \sqrt{2}\right) / 2}\right)+$

$$
\operatorname{Re}\left(\left(\varphi_{8}^{\prime}\right)^{\left(a k_{47}^{\prime}(q \sqrt{2}+2) / 2\right)}\right)=2+\operatorname{Re}\left(\left(\varphi_{8}^{\prime}\right)^{\left(a q k_{48} \sqrt{2}\right) / 2}\right)+\operatorname{Re}\left(\left(\varphi_{8}^{\prime}\right)^{\left(a k_{48}(q \sqrt{2}+2) / 2\right)}\right) .
$$

This implies that either $\operatorname{Re}\left(\left(\varphi_{8}^{\prime}\right)^{\left(q k_{47} \sqrt{2}\right) / 2}\right)=1$ or $\operatorname{Re}\left(\left(\varphi_{8}^{\prime}\right)^{\left(k_{47}(q \sqrt{2}+2) / 2\right)}\right)=$ 1. In both the cases, we obtain that $q^{2}+q \sqrt{2}+1$ divides $k_{47}$, which is a contradiction.

(2) Take $\psi_{2}$. Here, we get a contradiction similar to that obtained for $\psi_{1}$.

(3) Take $\psi_{3}=\chi_{2}+\chi_{4}+\chi_{10}+\chi_{11}+\chi_{13}+\chi_{17}+\chi_{20}+\chi_{47}\left(k_{47}\right)+\chi_{49}\left(k_{49}, l_{49}\right)$. Comparing the values at the classes $C_{42}(1)$ and $C_{43}(1)$ we obtain

$$
\operatorname{Re}\left(\left(\varphi_{8}^{\prime}\right)^{\left(q k_{47} \sqrt{2}\right) / 2}\right)+\operatorname{Re}\left(\left(\varphi_{8}^{\prime}\right)^{\left(k_{47}(q \sqrt{2}+2) / 2\right)}\right)=2 .
$$

This implies $\operatorname{Re}\left(\left(\varphi_{8}^{\prime}\right)^{\left(q k_{47} \sqrt{2}\right) / 2}\right)=1$, and hence $q^{2}+q \sqrt{2}+1$ divides $k_{47}$, which is a contradiction.

(4) Take $\psi_{4}$. Here, we get a contradiction similar to that obtained for $\psi_{3}$.

Observe that the group $G={ }^{2} F_{4}(2)^{\prime}$ has no reducible $S y l_{2}$-vanishing characters of degree $|G|_{2}$. On the other hand, it has four reducible $S y l_{2}$-vanishing characters of degree $2|G|_{2}$. In the notation of [3]:

(1) $\phi_{1}=2 \chi_{21}$;

(2) $\phi_{2}=2 \chi_{22}$;

(3) $\phi_{3}=\chi_{21}+\chi_{22}$;

(4) $\phi_{4}=\chi_{2}+\chi_{3}+\chi_{4}+\chi_{5}+2 \chi_{6}+\chi_{10}+\chi_{11}+\chi_{16}+\chi_{17}+\chi_{20}$.

The three characters $\phi_{1}, \phi_{2}, \phi_{3}$ are 2-vanishing, whereas the character $\phi_{4}$ is not. This can be easily proved by using the character table of $G$.

\section{The trivial COnstituent of a $p$-VAnishing Character}

Our next target is to prove that $1_{G}$ occurs as a constituent of every reducible $p$ vanishing character of degree $|G|_{p}$, if $G$ is quasi-simple and admits such characters. This turns out to be a rather non-trivial task. We observe that for some groups there is a stronger version of this result, see [24], Proposition 6.2 and Remark following it: 
Proposition 8.1. Let $G=S L(n, q), n>2$, or $G=E_{i}(q), i=6,7,8$. Let $\chi \neq S t$ be a Syl $l_{p}$-vanishing character of $G$ of degree $|G|_{p}$. Then $\left(\chi, 1_{G}\right)=1$.

Note that the above statement is not valid for symplectic and special unitary groups, as we already seen.

The groups of rank 2 have been analyzed in the previous section. We summarize the results about these groups in the following.

Proposition 8.2. Let $G$ be a Chevalley group of $B N$-pair rank $\ell=2$. Let $\chi \neq S t$ be a p-vanishing character of $G$ of degree $|G|_{p}$. Then $\left(\chi, 1_{G}\right)=1$ and either $G=$ $S L(3, q)$ or $G=S p(4, q)$ with $7 \mid(q+1)$.

Proof. See Lemmas 7.5, 7.6, 7.7, 7.8, 7.9, 7.11, 7.12 and 7.13,

In order to deal with groups with BN-pair rank $\ell>2$, we begin with some technical results.

Proposition 8.3. Let $\chi$ be a character of $G$. Set $\lambda=\left.\left(\bar{\chi}_{L_{J}}\right)\right|_{G_{J}}$ and $\lambda^{\prime}=\left.\left(\bar{\chi}_{L_{K}}\right)\right|_{G_{K}}$ with $J \cup K=I_{\ell}$.

(1) Suppose that $\lambda=S t_{G_{J}}$ and $\left({\overline{\lambda^{\prime}}}_{T_{K}}, 1_{T_{K}}\right)_{T_{K}}>0$. Then $\bar{\chi}_{L_{J}}=S t_{L_{J}}$.

(2) Suppose that $\bar{\chi}_{T}=1_{T}$ and that $\left(\lambda, 1_{G_{J}}\right)_{G_{J}}>0$. Then $\left(\bar{\chi}_{L_{J}}, 1_{L_{J}}\right)_{L_{J}}=1$.

(3) Suppose that $\left(\lambda, 1_{G_{J}}\right)_{G_{J}}>0$ and $\left(\lambda^{\prime}, 1_{G_{K}}\right)_{G_{K}}>0$. In addition, suppose

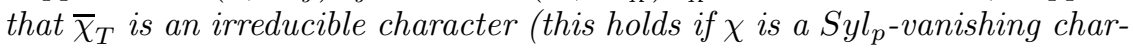
acter of degree $|G|_{p}$ ). Then, $1_{L_{J}}$ (resp. $1_{L_{K}}$ ) is a constituent of $\bar{\chi}_{L_{J}}$ (resp. $\left.\bar{\chi}_{L_{K}}\right)$ and $\bar{\chi}_{T}=1_{T}$.

Proof. (1) Note that $1_{T_{J}}$ coincides with the truncation of $S t_{G_{J}}$ to $T_{J}$. By Lemma $6.8(1), \bar{\chi}_{T}$ is a linear character (as so is $\beta_{J}$ ), and then ${\overline{\lambda^{\prime}}}_{T_{K}}$ is a linear character. So $\left.\left(\bar{\chi}_{T}\right)\right|_{T_{K}}=1_{T_{K}}$, again by Lemma 6.8. Therefore, $\bar{\chi}_{T}=1_{T}$ by Lemma 6.7.

Since $\lambda=S t_{G_{J}}$, the character $\bar{\chi}_{L_{J}}$ is irreducible of degree $\left|L_{J}\right|_{p}=\left|G_{J}\right|_{p}$, and by Lemma 6.5 this character is $S t_{L_{J}}$.

(2) By Frobenius reciprocity, $\left(\lambda, 1_{G_{J}}\right)_{G_{J}}=\left(\bar{\chi}_{L_{J}}, 1_{G_{J}}^{L_{J}}\right)_{L_{J}}>0$. Since $L_{J} / G_{J}$ is abelian, this implies that $\bar{\chi}_{L_{J}}$ has an irreducible constituent $\mu$, say, such that $\mu(g)=$ 1 for all $g \in G_{J}$. We show that actually $\mu=1_{L_{J}}$. Indeed, the character $\bar{\chi}_{T}=1_{T}$ contains the character $\bar{\mu}_{T}$, and, obviously, $\bar{\mu}_{T}=\left.\mu\right|_{T}$. Therefore, $\bar{\mu}_{T}=\left.\mu\right|_{T}=1_{T}$.

As $L_{J}=G_{J} T$, it follows that $\mu=1_{L_{J}}$. Furthermore, since $\bar{\chi}_{T}=1_{T}$, we have $\left(\bar{\chi}_{L_{J}}, 1_{L_{J}}\right)_{L_{J}}=1$.

(3) First note that if $\chi$ is a $S y l_{p}$-vanishing character of degree $|G|_{p}$, then $\left(\bar{\chi}_{T}\right)(1)=$ $\left(\left.\chi\right|_{U}, 1_{U}\right)=1$. Now, since $1_{G_{J}}$ is a constituent of $\lambda$, it follows that $1_{U \cap L_{J}}$ is a constituent of $\left.\lambda\right|_{U \cap L_{J}}$. This implies that $1_{T_{J}}$ is a constituent of $\bar{\lambda}_{T_{J}}$. By Lemma 6.8, $\bar{\lambda}_{T_{J}}$ coincides with the linear character $\left.\left(\bar{\chi}_{T}\right)\right|_{T_{J}}$. So $\bar{\lambda}_{T_{J}}=1_{T_{J}}$ and, similarly, $\bar{\lambda}_{T_{K}}=1_{T_{K}}$. By Lemma 6.7, $\bar{\chi}_{T}=1_{T}$. Now the result follows from the previous item.

Remark 8.4. It follows from (1) that if $\lambda=S t_{G_{J}}$ and $\lambda^{\prime}=S t_{G_{K}}$ then $\bar{\chi}_{L_{J}}=S t_{L_{J}}$.

Corollary 8.5. Let $G$ be a group of $B N$-pair rank $\ell>2$, and let $J, K$ be distinct subsets of $I_{\ell}$ such that $J \cup K=I_{\ell}$ and $J \cap K \neq \emptyset$. Let $\chi$ be a Syl $l_{p}$-vanishing character of $G$ and set $\lambda=\left.\left(\bar{\chi}_{L_{J}}\right)\right|_{G_{J}}$ and $\lambda^{\prime}=\left.\left(\bar{\chi}_{L_{K}}\right)\right|_{G_{K}}$. If $\lambda=S t_{G_{J}}$ then $\left(\lambda^{\prime}, 1_{G_{K}}\right)=0$.

Proof. Assume $\left(\lambda^{\prime}, 1_{G_{K}}\right)>0$. By Proposition $8.3(1), \bar{\chi}_{L_{J}}=S t_{L_{J}}$. Hence, $\bar{\chi}_{L_{J \cap K}}=$ $S t_{L_{J \cap K}}$ and $\bar{\chi}_{T}=1_{T}$. Now, by Proposition 8.3(2) we obtain $\left(\bar{\chi}_{L_{K}}, 1_{L_{K}}\right)=1$ and so $\bar{\chi}_{L_{J \cap K}}$ contains a linear character, a contradiction. 
We may prove now the following.

Theorem 8.6. Let $G$ be a Chevalley group and $\chi$ be a p-vanishing character of $G$ of degree $|G|_{p}$. Then either $\chi=$ St or $\left(\chi, 1_{G}\right)=1$.

Proof. We prove this theorem by induction on the BN-pair rank $\ell$ of $G$. We already proved that this theorem is true for $\ell=1$ (Proposition 6.6) and for $\ell=2$ (Proposition 8.2). Assume $\ell>2$. Take two subsets $J, K$ of $I_{\ell}$ such that $I_{\ell}=J \cup K$, $J \cap K \neq \emptyset$ and $|K|=2$. Set $\lambda=\left.\left(\bar{\chi}_{L_{J}}\right)\right|_{G_{J}}$ and $\lambda^{\prime}=\left.\left(\bar{\chi}_{L_{K}}\right)\right|_{G_{K}}$. Hence, by the induction hypothesis, we have three possibilities:

(a) $\lambda=S t_{G_{J}}$ and $\lambda^{\prime}=S t_{G_{K}}$;

(b) either $\lambda=S t_{G_{J}}$ and $\left(\lambda^{\prime}, 1_{G_{K}}\right)=1$ or $\left(\lambda, 1_{G_{J}}\right)=1$ and $\lambda^{\prime}=S t_{G_{K}}$;

(c) $\left(\lambda, 1_{G_{J}}\right)=1$ and $\left(\lambda^{\prime}, 1_{G_{K}}\right)=1$.

If (a) holds then $\chi=S t$ by Propositions 6.16 and 8.3(1). Case (b) is excluded by Corollary 8.5. If (c) holds, then $\left(\bar{\chi}_{L_{J}}, 1_{L_{J}}\right)=\left(\bar{\chi}_{L_{K}}, 1_{L_{K}}\right)=1$ by Proposition 8.3(3). Hence, Proposition 6.16 implies $\left(\chi, 1_{G}\right)=1$.

\section{Some Chevalley groups of higher Rank}

The results of the previous sections are insufficient to start induction. To set up the basis of induction, we additionally need to perform computational work for $H \in\{G L(n, q), n=4,5,6, C S p(6, q)\}$ in spirit of that done for groups of rank 2 . Our computations heavily use material available in the CHEvIE package. Note that CHEVIE does not provide information for groups $H=\operatorname{Spin}(n, q), n=7,9,11$ and $H=\operatorname{Spin}^{+}(n, q), n=8,10$, so for these groups $p$-vanishing characters of degree $|H|_{p}$ remain unknown.

9.1. Groups $H=G L(4, q), G L(5, q)$ and $G L(6, q)$. We apply Procedure 4.5 for these three groups. If $H=G L(4, q)$, we obtain in step (1) the $S y l_{p}$-decompositions of Table 11.12. B and $\Delta_{H}=\{3,4,5,14,18,22,23\}$. Note that $\chi_{22}$ and $\chi_{23}$ are the only regular characters in this set. In step (2), let $\psi$ be a $S y l_{p}$-vanishing character of $H$ of degree $|H|_{p}$, whose constituents lie in $\Delta_{H}$.

(1) Suppose that $\chi_{22}$ is a constituent of $\psi$. Then, by degree reasons, the other constituents of $\psi$ must belong to the set $\left\{\chi_{h} \mid h=4,5,14\right\}$. Looking at the class $C_{5}(q-1)$ we easily obtain a contradiction.

(2) Suppose that $\chi_{23}$ is a constituent of $\psi$. Then the other constituents of $\psi$ must belong to the set $\left\{\chi_{h} \mid h=3,4,5,14,18\right\}$. Looking at the class $C_{3}(q-1)$, the character $\chi_{18}$ must be a constituent of $\psi$. Now, the character values on the class $C_{2}(q-1)$ imply that $\chi_{14}$ is another constituent of $\psi$. So, we obtain a unique $S y l_{p}$-vanishing character of degree $|H|_{p}: \psi_{1}=$ $\chi_{5}+\chi_{14}+\chi_{18}+\chi_{23}$.

In step (3) we get all $S y l_{p}$-vanishing characters of $H$ of degree $|H|_{p}$ (see Table 11.12. $\mathrm{C}$ ).

Lemma 9.1. Let $H=G L(4, q)$. Then $H$ admits reducible $p$-vanishing characters $\psi$ of degree $|H|_{p}$ if and only if $3 \mid(q+1)$. In this case $H$ admits exactly $(q-1)\left(q^{2}-\delta\right) / 4$ characters $\psi$ with $\delta=\frac{1-(-1)^{q}}{2}$, where

$$
\psi=\chi_{5}(k) \cdot\left(1_{H}+\chi_{14}(a)+\chi_{18}(0, a)+\chi_{23}\left(\left(q^{2}-1\right) b\right)\right),
$$

$\chi_{5}(k)$ is a linear character of $H, a=(q+1) / 3$ and $b \in \mathbb{Z}_{q^{2}+1} \backslash\{0\}$. 
Proof. Up to linear characters of $H$ we have the following possibilities for $\psi$ :

(1) $\psi_{1}=1_{H}+\chi_{14}\left(k_{14,1}\right)+\chi_{18}\left(k_{18,1}, k_{18,2}\right)+\chi_{23}\left(k_{23,1}\right)$, with $(q-1)$ dividing $2 k_{14,1},\left(2 k_{18,1}+k_{18,2}\right)$ and $k_{23,1}$ (so $\left.k_{23,1}=(q-1) k_{23,1}^{\prime}\right)$. On the classes $C_{7}\left(i_{1}, i_{2}\right)$ we obtain $\zeta_{1}^{\left(i_{2}-i_{1}\right) k_{18,1}}=1$, i.e. $k_{18,1}=0$ and $k_{18,2}=(q-1) k_{18,2}^{\prime}$. Now, on the classes $C_{12}\left(i_{1}, i_{2}\right)$ we have $\zeta_{1}^{\left(i_{1}+i_{2}\right) k_{14,1}}=1$, so $k_{14,1}=(q-$ 1) $k_{14,1}^{\prime}$. On the classes $C_{14}\left(i_{1}\right)$ we have

$$
1+\operatorname{Re}\left(\xi_{1}^{2 i_{1} k_{14,1}^{\prime}}\right)=\operatorname{Re}\left(\xi^{i_{1} k_{18,2}^{\prime}}\right)+\operatorname{Re}\left(\xi_{1}^{i_{1} k_{23,1}^{\prime}}\right),
$$

and on the classes $C_{18}\left(i_{1}, i_{2}\right)$ we have

$$
\operatorname{Re}\left(\xi_{1}^{i_{2} k_{14,1}^{\prime}}\right)=\operatorname{Re}\left(\xi_{1}^{i_{2} k_{18,2}^{\prime}}\right) .
$$

This holds if and only if 3 divides $q+1, \xi_{1}^{k_{14,1}^{\prime}}, \xi_{1}^{k_{18,2}^{\prime}} \in\left\{\omega, \omega^{2}\right\}$ and $(q+1) \mid$ $k_{23,1}^{\prime}$.

(2) $\psi_{2}=1_{H}+\chi_{13}\left(k_{13,1}\right)+\chi_{18}\left(k_{18,1}, k_{18,2}\right)$. On the class $C_{14}(q-1)$ we obtain $\operatorname{Re}\left(\xi_{1}^{k_{18,2}}\right)=1$, i.e. $(q+1) \mid k_{18,2}$, a contradiction.

If $H=G L(5, q)$, with step (1) we obtain the $S y l_{p}$-decompositions of Table 11.13B $\mathrm{B}$ and $\Delta_{H}=\{5,6,7,23,24,30,36,41,42,43\}$. Note that $\chi_{41}, \chi_{42}$ and $\chi_{43}$ are the only regular characters of this set. In step (2), let $\psi$ be a $S y l_{p^{-}}$vanishing character of $H$ of degree $|H|_{p}$, whose constituents lie in $\Delta_{H}$.

(1) Let $\chi_{41}$ be a constituent of $\psi$. By degree reasons, the other constituents of $\psi$ must belong to the set $\left\{\chi_{h} \mid h=5,6,7,24,30,36\right\}$. Looking at the class $C_{6}(q-1)$, we observe that $\psi$ must have at least $q-1$ constituents of type $\chi_{24}$, in contradiction with Lemma 3.12 .

(2) Let $\chi_{42}$ be a constituent of $\psi$. Then the other constituents of $\psi$ must belong to the set $\left\{\chi_{h} \mid h=5,6,7,24,30\right\}$. Using the class $C_{5}(q-1)$, we conclude that $\chi_{24}$ must be a constituent of $\psi$. Now, using the class $C_{4}(q-1)$, with these constituents we obtain a unique $S y l_{p}$-vanishing character of degree $|H|_{p}: \psi_{1}=\chi_{7}+\chi_{24}+\chi_{30}+\chi_{42}$.

(3) Let $\chi_{43}$ be a constituent of $\psi$. Then the other constituents of $\psi$ must belong to the set $\left\{\chi_{h} \mid h=5,6,7,24,30,36\right\}$. Looking at the classes $C_{4}(q-1)$ and $C_{7}(q-1)$, we observe that both $\chi_{24}$ and $\chi_{30}$ must be constituents of $\psi$. However, using $C_{7}(q-1)$, it follows that $\psi$ must admit another constituent of type $\chi_{24}$, which contradicts Lemma 3.12 .

In step (3) we get all $S y l_{p}$-vanishing characters of $H$ of degree $|H|_{p}$ (see Table 11.13 - C).

Lemma 9.2. Let $H=G L(5, q)$. Then $H$ admits reducible p-vanishing characters $\psi$ of degree $|H|_{p}$ if and only if $3 \mid(q+1)$. In this case, there are at most $q^{2}(q-1) / 2$ characters

$$
\psi=\chi_{7}(k) \cdot\left(1_{H}+\chi_{24}(0, a)+\chi_{30}(a, 0)+\chi_{42}\left(\left(q^{2}-1\right) b, 0\right)\right),
$$

where $\chi_{7}(k)$ is a linear character of $H, a=(q+1) / 3$ and $b \in \mathbb{Z}_{q^{2}+1} \backslash\{0\}$.

Proof. Modulo linear characters of $H$ we have the following possibilities: 
(1) $\psi_{1}=1_{H}+\chi_{24}\left(k_{24,1}, k_{24,2}\right)+\chi_{30}\left(k_{30,1}, k_{30,2}\right)+\chi_{42}\left(k_{42,1}, k_{42,2}\right)$, where $q-1$ must divide $3 k_{24,1}+k_{24,2}, 2 k_{30,1}+k_{30,2}$ and $k_{41,1}+k_{42,2}$. On the classes $C_{36}\left(i_{1}, i_{2}\right)$ we have $\zeta_{1}^{\left(i_{2}-3 i_{1}\right) k_{24,1}}=1$. So $k_{24,1}=0$ and $k_{24,2}=(q-1) k_{24,2}^{\prime}$. Now, on the classes $C_{15}\left(i_{1}, i_{2}\right)$ we have $\zeta_{1}^{\left(i_{2}-i_{1}\right) k_{30,1}}=1$, i.e. $k_{30,1}=(q-$ 1) $k_{30,1}^{\prime}$ and $k_{30,2}=0$. Next, on $C_{9}\left(i_{1}, i_{2}\right)$ we have $\zeta_{1}^{\left(i_{2}-i_{1}\right) k_{42,2}}=1$, i.e. $k_{42,2}=0$ and $k_{42,1}=(q-1) k_{42,1}^{\prime}$. On the classes $C_{23}\left(i_{1}, i_{2}\right)$ we have $\operatorname{Re}\left(\xi_{1}^{i_{2} k_{24,2}^{\prime}}\right)=\operatorname{Re}\left(\xi_{1}^{i_{2} k_{30,1}^{\prime}}\right)$. Finally, consider the classes $C_{30}\left(i_{1}, i_{2}\right)$ where we have

$$
1+\operatorname{Re}\left(\xi_{1}^{2 i_{1} k_{30,1}^{\prime}}\right)=\operatorname{Re}\left(\xi_{1}^{i_{1} k_{30,1}^{\prime}}\right)+\operatorname{Re}\left(\xi_{1}^{i_{1} k_{42,1}^{\prime}}\right) .
$$

This means that $(q+1) \mid k_{42,1}^{\prime}$ and $\xi_{1}^{k_{30,1}^{\prime}}=\omega^{ \pm 1}$. Thus, 3 must divide $q+1$. (2) $\psi_{2}=1_{H}+\chi_{24}\left(k_{24,1}, k_{24,2}\right)+\chi_{29}\left(k_{29,1}, k_{29,2}\right)$. On the class $C_{30}(q-1, q-1)$ we obtain $\operatorname{Re}\left(\xi_{1}^{k_{24,2}}\right)=1$, which implies that $q+1$ divides $k_{24,2}$, a contradiction.

If $H=G L(6, q)$, with step (1) we obtain the $S y l_{p}$-decompositions of Table 11.14 $\mathrm{B}$ and $\Delta_{H}=\{6,7,9,10,11,39,40,48,49,50,68,70,78,79,99,107,110,111,112\}$. Note that $\chi_{107}, \chi_{110}, \chi_{111}$ and $\chi_{112}$ are the only regular characters in this set. In step (2), let $\psi$ be a $S y l_{p}$-vanishing character of $H$ of degree $|H|_{p}$, whose constituents lie in $\Delta_{H}$.

(1) Suppose that $\chi_{107}$ is a constituent of $\psi$. By degree reasons, the other constituents of $\psi$ must belong to the set $\left\{\chi_{h} \mid h=7,9,10,11,40,50\right\}$. Then, using the class $C_{3}(q-1)$ we get a contradiction.

(2) Suppose that $\chi_{110}$ is a constituent of $\psi$. Then, by degree reasons, the other constituents of $\psi$ must belong to the set $\left\{\chi_{h} \mid h=6,7,9,10,11,39,40,48,49\right.$, $50,68,70,79,99\}$. Then, using the class $C_{3}(q-1)$, we conclude that some constituent of $\psi$ must be of type $\chi_{99}$. With some computations, we observe that the only possibility remained is the $S y l_{p}$-character $\psi_{1}=$ $\chi_{11}+\chi_{40}+\chi_{50}+\chi_{68}+\chi_{99}+\chi_{110}$.

(3) Suppose that $\chi_{111}$ is a constituent of $\psi$. By degree reasons, the other constituents of $\psi$ must belong to the set $\left\{\chi_{h} \mid h=6,7,9,10,11,39,40,48\right.$, $49,50,68,70,79\}$. Using the class $C_{11}(q-1)$, we see that either $\chi_{40}$ or $\chi_{50}$ must be a constituent of $\psi$. In both the cases, we obtain a contradiction.

(4) Suppose that $\chi_{112}$ is a constituent of $\psi$. Then, by degree reasons, the other constituents of $\psi$ must belong to the set $\left\{\chi_{h} \mid h=6,7,9,10,11,39,40,48,49\right.$, $50,68,70,79,99\}$. Using the class $C_{5}(q-1)$, we observe that $\chi_{39}$ must be a constituent of $\psi$. With some computations we again obtain a contradiction.

In step (3) we obtain the list of all $S y l_{p}$-vanishing characters of $H$ of degree $|H|_{p}$ (see Table 11.14. C).

Lemma 9.3. Let $H=G L(6, q)$. Then, both $H$ and $G=H^{\prime}$ have no reducible p-vanishing character of degree $|H|_{p}$.

Proof. Suppose the contrary. Modulo linear characters of $H$, we have to inspect the following cases.

(1) $\psi_{1}=1_{H}+\chi_{40}\left(k_{40,1}\right)+\chi_{50}\left(k_{50,1}, k_{50,2}\right)+\chi_{68}\left(k_{68,1}, k_{68,2}\right)+\chi_{99}\left(k_{99,1}, k_{99,2}\right)+$ $+\chi_{110}\left(k_{110,1}, k_{110,2}\right)$. On the classes $C_{40}\left((q-1) i_{1}^{\prime}\right)\left(i_{1}^{\prime}=1,2\right)$ we have $1+\operatorname{Re}\left(\xi_{1}^{2 i_{1}^{\prime} k_{68,2}}\right)+\operatorname{Re}\left(\xi_{1}^{i_{1}^{\prime}\left(k_{110,1}+k_{110,2}\right)}\right)+\operatorname{Re}\left(\xi_{1}^{i_{1}^{\prime}\left(k_{110,1}-k_{110,2}\right)}\right)=\operatorname{Re}\left(\xi_{1}^{i_{1}^{\prime} k_{40,1}}\right)+$ 
$\operatorname{Re}\left(\xi_{1}^{3 i_{1}^{\prime} k_{40,1}}\right)+\operatorname{Re}\left(\xi_{1}^{i_{1}^{\prime} k_{50,2}}\right)+$

$+\operatorname{Re}\left(\xi_{1}^{i_{1}^{\prime} k_{99,2}}\right)$. Next, on the classes $C_{39}\left((q-1) i_{1}^{\prime}\right)\left(i_{1}^{\prime}=1,2\right)$ we get $1+\operatorname{Re}\left(\xi_{1}^{2 i_{1}^{\prime} k_{68,2}}\right)=\operatorname{Re}\left(\xi_{1}^{i_{1}^{\prime} k_{1,40}}\right)+\operatorname{Re}\left(\xi_{1}^{i_{1}^{\prime} k_{50,2}}\right)$. This implies that $q+1$ divides either $k_{40,1}$ or $k_{50,2}$. In both the cases, we have a contradiction.

(2) $\psi_{2}=1_{H}+\chi_{40}\left(k_{40,1}\right)+\chi_{50}\left(k_{50,1}, k_{50,2}\right)+\chi_{67}\left(k_{67,1}, k_{67,2}\right)+\chi_{110}\left(k_{110,1}, k_{110,2}\right)$. On the classes $C_{40}\left((q-1) i_{1}^{\prime}\right)\left(i_{1}^{\prime}=1,2\right)$ we have

$\operatorname{Re}\left(\xi_{1}^{i_{1}^{\prime} k_{40,1}}\right)+\operatorname{Re}\left(\xi_{1}^{3 i_{1}^{\prime} k_{40,1}}\right)+\operatorname{Re}\left(\xi_{1}^{i_{1}^{\prime}} k_{50,2}\right)=1+\operatorname{Re}\left(\xi_{1}^{i_{1}^{\prime}\left(k_{110,1}+k_{110,2}\right)}\right)+\operatorname{Re}\left(\xi_{1}^{i_{1}^{\prime}\left(k_{110,1}-k_{110,2}\right)}\right)$.

Furthermore, on the classes $C_{39}\left((q-1) i_{1}^{\prime}\right)\left(i_{1}^{\prime}=1,2\right)$ we get $1+\operatorname{Re}\left(\xi_{1}^{2 i_{1}^{\prime} k_{67,1}}\right)=$ $\operatorname{Re}\left(\xi_{1}^{i_{1}^{\prime} k_{40,1}}\right)+\operatorname{Re}\left(\xi_{1}^{i_{1}^{\prime} k_{50,2}}\right)$. This implies that $q+1$ divides either $k_{40,1}$ or $k_{50,2}$. In both the cases, we have a contradiction.

(3) $\psi_{3}=1_{H}+\chi_{38}\left(k_{38,1}\right)+\chi_{50}\left(k_{50,1}, k_{50,2}\right)+\chi_{68}\left(k_{68,1}, k_{68,2}\right)+\chi_{99}\left(k_{99,1}, k_{99,2}\right)$.

On the class $C_{49}(q-1, q-1)$ we have $\operatorname{Re}\left(\xi_{1}^{k_{50,2}}\right)=\operatorname{Re}\left(\xi_{1}^{k_{68,2}}\right)$. On the classes $C_{40}\left((q-1) i_{2}^{\prime}\right)\left(i_{2}^{\prime}=1,2\right)$ we have $1+\operatorname{Re}\left(\xi_{1}^{i_{2}^{\prime} k_{99,2}}\right)=\operatorname{Re}\left(\xi_{1}^{i_{2}^{\prime} k_{50,2}}\right)+$ $\operatorname{Re}\left(\xi_{1}^{2 i_{2}^{\prime} k_{68,2}}\right)$, which implies $\operatorname{Re}\left(\xi_{1}^{2 k_{68,2}}\right)=1$ and $\operatorname{Re}\left(\xi_{1}^{k_{99,2}}\right)=\operatorname{Re}\left(\xi_{1}^{k_{50,2}}\right)$. On the classes $C_{39}\left((q-1) i_{1}^{\prime}\right)\left(i_{2}^{\prime}=1,2\right)$ we have $\left(q^{2}+1\right)\left(1+\operatorname{Re}\left(\xi_{1}^{2 i_{2}^{\prime} k_{68,2}}\right)=\right.$ $\left(q^{2}+1\right) \operatorname{Re}\left(\xi_{1}^{i_{1}^{\prime} k_{50,2}}\right)+q^{2} \operatorname{Re}\left(\xi_{1}^{i_{1}^{\prime} k_{38,1}}\right)+\operatorname{Re}\left(\xi_{1}^{i_{1}^{\prime} k_{99,1}}\right)$.

So $\left(q^{2}+2\right) \operatorname{Re}\left(\xi_{1}^{i_{1}^{\prime} k_{50,2}}\right)+q^{2} \operatorname{Re}\left(\xi_{1}^{i_{1}^{\prime} k_{38,1}}\right)=2\left(q^{2}+1\right)$ which implies $\operatorname{Re}\left(\xi_{1}^{k_{88,1}}\right)=$ 1, i.e. $(q+1) \mid k_{38,1}$, a contradiction.

(4) $\psi_{4}=1_{H}+\chi_{40}\left(k_{40,1}\right)+\chi_{50}\left(k_{50,1}, k_{50,2}\right)+\chi_{68}\left(k_{68,1}, k_{68,2}\right)+\chi_{98}\left(k_{98,1}, k_{98,2}\right)$. On the classes $C_{99}(q-1, q-1)$ we have $\operatorname{Re}\left(\xi_{1}^{k_{40,1}}\right)=\operatorname{Re}\left(\xi_{1}^{k_{68,2}}\right)$, and on the classes $C_{49}\left(q-1,(q-1) i_{2}^{\prime}\right)\left(i_{2}^{\prime}=1,2\right)$ we have $q-1+\operatorname{Re}\left(\xi_{1}^{i_{2}^{\prime} k_{50,2}}\right)=$ $(q-1) \operatorname{Re}\left(\xi_{1}^{i_{2}^{\prime} k_{40,1}}\right)+\operatorname{Re}\left(\xi_{1}^{i_{2}^{\prime} k_{68,2}}\right)$. Hence, $q-1+\operatorname{Re}\left(\xi_{1}^{i_{2}^{\prime} k_{50,2}}\right)=q \operatorname{Re}\left(\xi_{1}^{i_{2}^{\prime} k_{40,1}}\right)$, which implies $\operatorname{Re}\left(\xi_{1}^{k_{40,1}}\right)=1$, i.e. $(q+1) \mid k_{40,1}$, a contradiction.

(5) $\psi_{5}=1_{H}+\chi_{38}\left(k_{38,1}\right)+\chi_{50}\left(k_{50,1}, k_{50,2}\right)+\chi_{67}\left(k_{67,1}, k_{67,2}\right)$. On the class $C_{40}(q-1)$ we have $\operatorname{Re}\left(\xi_{1}^{k_{50,2}}\right)=1$, i.e. $(q+1) \mid k_{50,2}$. However, this contradicts the conditions on the parameters.

Since all the conjugacy classes that we have considered belong to $G=H^{\prime}$, if follows from Proposition 6.2 that $G$ also has no $p$-vanishing character of degree $|G|_{p}$.

9.2. Groups $H=C S p(6, q)$ with $q$ odd. Applying Procedure 4.5, in step (1) we obtain the $S y l_{p}$-decompositions of Table11.15\}-B and $\Delta_{H}=\{1,4,5,7,10,11,18,36$, $45,50,51,65,71,97,98\}$. For step (2), we note that the only regular characters belonging to this set are $\chi_{97}$ and $\chi_{98}$. Let $\psi$ be a $S y l_{p}$-vanishing character whose constituents lie in $\Delta_{H}$.

(i) Let $\chi_{97}$ be a constituent of $\psi$. Then, by degree reasons, the other constituents of $\psi$ must belong to the set $\left\{\chi_{h} \mid h=1,4,5,18,50,71\right\}$. Using the class $C_{10}(q-1), \psi$ must have at least one constituent of type $\chi_{18}$. This easily leads to a contradiction.

(ii) Let $\chi_{98}$ be a constituent of $\psi$. Then, using the class $C_{8}(q-1)$, we observe that another constituent of $\psi$ must be either of type $\chi_{45}$, or $\chi_{50}$, or $\chi_{51}$. 
We get, with some efforts, the following $S y l_{p}$-vanishing characters:

$$
\begin{aligned}
& \psi_{1}=\chi_{1}+\chi_{5}+\chi_{11}+\chi_{18}+\chi_{45}+\chi_{45}^{\prime}+\chi_{45}^{\prime \prime}+\chi_{50}+\chi_{51}+\chi_{65}+\chi_{71}+\chi_{98}, \\
& \psi_{2}=\chi_{1}+\chi_{11}+\chi_{11}^{\prime}+\chi_{18}+\chi_{45}+\chi_{45}^{\prime}+\chi_{45}^{\prime \prime}+\chi_{45}^{\prime \prime}+\chi_{50}+\chi_{65}+\chi_{71}+\chi_{98}, \\
& \psi_{3}=\chi_{1}+\chi_{5}+\chi_{5}^{\prime}+\chi_{18}+\chi_{45}+\chi_{45}^{\prime}+\chi_{50}+\chi_{51}+\chi_{51}^{\prime}+\chi_{65}+\chi_{71}+\chi_{98}, \\
& \psi_{4}=\chi_{5}+\chi_{10}+\chi_{11}+\chi_{11}^{\prime}+\chi_{18}+\chi_{45}+\chi_{45}^{\prime}+\chi_{45}^{\prime \prime}+\chi_{50}+\chi_{71}+\chi_{98}, \\
& \psi_{5}=\chi_{5}+\chi_{5}^{\prime}+\chi_{10}+\chi_{11}+\chi_{18}+\chi_{45}+\chi_{45}^{\prime}+\chi_{50}+\chi_{51}+\chi_{71}+\chi_{98}, \\
& \psi_{6}=\chi_{5}+\chi_{5}^{\prime}+\chi_{5}^{\prime \prime}+\chi_{10}+\chi_{18}+\chi_{45}+\chi_{50}+\chi_{51}+\chi_{51}^{\prime}+\chi_{71}+\chi_{98} .
\end{aligned}
$$

Now, in step (3) we obtain the list of all $S y l_{p}$-vanishing characters of $H$ of degree $|H|_{p}$ (see Table 11.15-C).

Lemma 9.4. Let $H=C S p(6, q)$, $q$ odd. Then both $H$ and $G=H^{\prime}$ have no reducible p-vanishing character of degree $|H|_{p}$.

Proof. Suppose the contrary. We consider the characters $\psi_{j}$ described in Table 11.15-C. By Lemma 7.9, a reducible $p$-vanishing character $\psi$ of $G$ of degree $|G|_{p}$ must satisfy $\left.\left(\bar{\psi}_{P_{J}}\right)\right|_{G_{J}}=1_{G}+\chi_{4}(a, b)+\chi_{6}(c)+\chi_{10}(d)$, where $G_{J} \cong S p(4, q)$. So it suffices to consider only the following cases:

(1) Take $\psi_{j}$ with $j \in\{55,56\}$. We obtain a contradiction with the value at the class $C_{26}(1,0)$.

(2) Take $\psi_{j}$ with $j \in\{58,59,61,62,63,64,65,66,67,70,71,74,75\}$. We obtain a contradiction with the values at the class $C_{25}(1,0)$.

(3) Take $\psi_{81}$. Using the class $C_{70}(1, q-1)$ we have $\operatorname{Re}\left(\xi_{1}^{k_{85,1}}\right)=\operatorname{Re}\left(\xi_{1}^{k_{65,1}}\right)$ and using the class $C_{72}\left(i_{1}, 1, q-1\right)$ we have $\operatorname{Re}\left(\xi_{1}^{k_{85,2}}\right)=\operatorname{Re}\left(\xi_{1}^{k_{41,1}}\right)$. On the classes $C_{74}\left(i_{1}, i_{2}, q-1\right)$ we have $\operatorname{Re}\left(\xi_{1}^{\left(k_{87,1}+k_{87,2}\right) i_{1}}\right)+\operatorname{Re}\left(\xi_{1}^{\left(k_{87,1}-k_{87,2}\right) i_{1}}\right)=$ $\operatorname{Re}\left(\xi_{1}^{2 k_{65,1} i_{1}}\right)+\operatorname{Re}\left(\xi_{1}^{k_{41,1} i_{1}}\right)$ for all $i_{1}=1, \ldots, \frac{q-1}{2}$. Using the classes $C_{68}\left(i_{1}, i_{2}\right.$, $q-1)$ we have $\operatorname{Re}\left(\xi_{1}^{i_{1} k_{87,1}}\right)+\operatorname{Re}\left(\xi_{1}^{i_{1} k_{87,2}}\right)=\operatorname{Re}\left(\xi_{1}^{i_{1} k_{65,1}}\right)+\operatorname{Re}\left(\xi_{1}^{i_{1} k_{41,1}}\right)$ for all $i_{1}=1, \ldots, \frac{q-1}{2}$. Using the classes $C_{38}\left(i_{1}, q-1\right)$ and the previous information, we obtain

$$
\begin{gathered}
\operatorname{Re}\left(\xi_{1}^{\left(k_{98,1}+k_{98,2}+k_{98,3}\right) i_{1}}\right)+\operatorname{Re}\left(\xi_{1}^{\left(k_{98,1}-k_{98,2}+k_{98,3}\right) i_{1}}\right)+ \\
+\operatorname{Re}\left(\xi_{1}^{\left(k_{98,1}-k_{98,2}-k_{98,3}\right) i_{1}}\right)+\operatorname{Re}\left(\xi_{1}^{\left(k_{98,1}+k_{98,2}-k_{98,3}\right) i_{1}}\right)= \\
=\operatorname{Re}\left(\xi_{1}^{\left(2 k_{85,1}+k_{85,2}\right) i_{1}}\right)+\operatorname{Re}\left(\xi_{1}^{\left(2 k_{85,1}-k_{85,2}\right) i_{1}}\right)+\operatorname{Re}\left(\xi_{1}^{k_{51,1} i_{1}}\right)+\operatorname{Re}\left(\xi^{k_{85,2} i_{1}}\right)
\end{gathered}
$$

for all $i_{1}=1, \ldots, \frac{q-1}{2}$. Using the classes $C_{37}\left(i_{1}, q-1\right)$ and the previous information, we obtain $1+\operatorname{Re}\left(\xi_{1}^{\left(2 k_{85,1}+k_{85,2}\right) i_{1}}\right)+\operatorname{Re}\left(\xi_{1}^{\left(2 k_{85,1}-k_{85,2}\right) i_{1}}\right)=$ $\operatorname{Re}\left(\xi_{1}^{2 k_{65,1} i_{1}}\right)+\operatorname{Re}\left(\xi_{1}^{k_{41,1} i_{1}}\right)+\operatorname{Re}\left(\xi_{1}^{3 k_{51,1} i_{1}}\right)$ for all $i_{1}=1, \ldots, \frac{q-1}{2}$. This implies that either $\xi_{1}^{2 k_{65,1}}=1$, or $\xi_{1}^{k_{41,1}}=1$, or $\xi_{1}^{3 k_{51,1}}=1$. In the first two cases, we get a contradiction with the conditions on the parameters $k_{65,1}$ and $k_{41,1}$. So $\xi_{1}^{3 k_{51,1}}=1$, i.e. $\xi_{1}^{k_{51,1}}=\omega^{ \pm 1}$. Furthermore, $\operatorname{Re}\left(\xi_{1}^{\left(2 k_{85,1}+k_{85,2}\right) i_{1}}\right)+\operatorname{Re}\left(\xi_{1}^{\left(2 k_{85,1}-k_{85,2}\right) i_{1}}\right)=\operatorname{Re}\left(\xi_{1}^{2 k_{65,1} i_{1}}\right)+\operatorname{Re}\left(\xi^{k_{41,1} i_{1}}\right)$.

First observe that

$$
\left\{\begin{array}{l}
\operatorname{Re}\left(\xi_{1}^{k_{85,1}}\right)=\operatorname{Re}\left(\xi_{1}^{k_{65,1}}\right) \\
\operatorname{Re}\left(\xi_{1}^{k_{85,2}}\right)=\operatorname{Re}\left(\xi_{1}^{k_{41,1}}\right) \\
\operatorname{Re}\left(\xi_{1}^{2 k_{85,1}+k_{85,2}}\right)+\operatorname{Re}\left(\xi_{1}^{2 k_{85,1}-k_{85,2}}\right)=\operatorname{Re}\left(\xi_{1}^{2 k_{65,1}}\right)+\operatorname{Re}\left(\xi_{1}^{k_{41,1}}\right) \\
\operatorname{Re}\left(\xi_{1}^{4 k_{85,1}+2 k_{85,2}}\right)+\operatorname{Re}\left(\xi_{1}^{4 k_{85,1}-2 k_{85,2}}\right)=\operatorname{Re}\left(\xi_{1}^{4 k_{65,1}}\right)+\operatorname{Re}\left(\xi_{1}^{2 k_{41,1}}\right)
\end{array} .\right.
$$


Setting $A=\operatorname{Re}\left(\xi_{1}^{k_{85,1}}\right)$ and $B=\operatorname{Re}\left(\xi_{1}^{k_{85,2}}\right)$, we obtain

$$
\left\{\begin{array}{l}
2\left(2 A^{2}-1\right) B=\left(2 A^{2}-1\right)+B \\
2\left(8 A^{4}-8 A^{2}+1\right)\left(2 B^{2}-1\right)=\left(8 A^{4}-8 A^{2}+1\right)+\left(2 B^{2}-1\right)
\end{array} .\right.
$$

Solving this system and remembering that $A \neq \pm 1$, we obtain that either

or

$$
\text { (a) } A=\frac{1 \pm \sqrt{5}}{4}, \quad B=-A
$$

$$
\text { (b) } A=\frac{-1 \pm \sqrt{5}}{4}, \quad B=A \text {. }
$$

Now, consider the following equations:

$$
\left\{\begin{array}{l}
\operatorname{Re}\left(\xi_{1}^{k_{87,1}}\right)+\operatorname{Re}\left(\xi_{1}^{k_{87,2}}\right)=\operatorname{Re}\left(\xi_{1}^{k_{65,1}}\right)+\operatorname{Re}\left(\xi_{1}^{k_{41,1}}\right) \\
\operatorname{Re}\left(\xi_{1}^{k_{87,1}+k_{87,2}}\right)+\operatorname{Re}\left(\xi_{1}^{k_{87,1}-k_{87,2}}\right)=\operatorname{Re}\left(\xi_{1}^{2 k_{65,1}}\right)+\operatorname{Re}\left(\xi_{1}^{k_{41,1}}\right) \\
\operatorname{Re}\left(\xi_{1}^{2 k_{87,1}}\right)+\operatorname{Re}\left(\xi_{1}^{2 k_{87,2}}\right)=\operatorname{Re}\left(\xi_{1}^{2 k_{65,1}}\right)+\operatorname{Re}\left(\xi_{1}^{2 k_{41,1}}\right)
\end{array}\right.
$$

Setting $X=\operatorname{Re}\left(\xi_{1}^{k_{87,1}}\right)$ and $Y=\operatorname{Re}\left(\xi_{1}^{k 87,2}\right)$, we obtain in case (a)

$$
\left\{\begin{array}{l}
2 X Y=-1 / 2 \\
X+Y=0 \\
X^{2}+Y^{2}=2 A^{2}
\end{array}\right.
$$

So $Y=-X, X^{2}=\frac{1}{4}$ and $\frac{1}{2}=2\left(\frac{1 \pm \sqrt{5}}{4}\right)^{2}$, an absurd. In case (b) we get

$$
\left\{\begin{array}{l}
2 X Y=-1 / 2 \\
X+Y=2 A \\
X^{2}+Y^{2}=2 A^{2}
\end{array}\right.
$$

This implies $A^{2}=-1 / 4$, again an absurd.

(4) Take $\psi_{82}$. Using the classes $C_{70}(1, q-1)$ and $C_{72}\left(i_{1}, q+1, q-1\right)$, we have $\operatorname{Re}\left(\xi_{1}^{k_{85,1}}\right)=\operatorname{Re}\left(\xi_{1}^{k_{65,1}}\right)$ and $\operatorname{Re}\left(\xi_{1}^{k_{85,2}}\right)=\operatorname{Re}\left(\xi_{1}^{k_{41,1}}\right)$. Now, considering also the class $C_{32}(q, q-1)$, we obtain $\operatorname{Re}\left(\xi_{1}^{k_{85,1}}\right)+\operatorname{Re}\left(\xi_{1}^{k_{85,2}}\right)+\operatorname{Re}\left(\xi_{1}^{k_{51,1}}\right)=$ $1+\operatorname{Re}\left(\xi_{1}^{k_{65,1}}\right)+\operatorname{Re}\left(\xi_{1}^{k_{41,1}}\right)$, whence $\operatorname{Re}\left(\xi_{1}^{k_{51,1}}\right)=1$, a contradiction.

(5) Take $\psi_{83}$. Using the class $C_{70}(1, q-1)$ we have $\operatorname{Re}\left(\xi_{1}^{k_{86,1}}\right)=\operatorname{Re}\left(\xi_{1}^{k_{65,1}}\right)$, and looking at the class $C_{72}\left(i_{1}, 1, q-1\right)$ we have $\operatorname{Re}\left(\xi_{1}^{k_{86,2}}\right)=\operatorname{Re}\left(\xi_{1}^{k_{41,1}}\right)$. Using the classes $C_{74}\left(i_{1}, i_{2}, q-1\right)$ we have $\operatorname{Re}\left(\xi_{1}^{\left(k_{87,1}+k_{87,2}\right) i_{1}}\right)+R e\left(\xi_{1}^{\left(k_{87,1}-k_{87,2}\right) i_{1}}\right)=$ $\operatorname{Re}\left(\xi_{1}^{2 k_{65,1} i_{1}}\right)+\operatorname{Re}\left(\xi_{1}^{k_{41,1} i_{1}}\right)$ for all $i_{1}=1,2,3$. Using the classes $C_{37}\left(i_{1}, q-\right.$ $1)$ and the previous information, we obtain $q+q \operatorname{Re}\left(\xi_{1}^{\left(2 k_{86,1}-k_{86,2}\right) i_{1}}\right)+$ $q \operatorname{Re}\left(\xi_{1}^{\left(2 k_{86,1}+k_{86,2}\right) i_{1}}\right)+(q-1) \operatorname{Re}\left(\xi_{1}^{k_{41,1} i_{1}}\right)=q \operatorname{Re}\left(\xi_{1}^{2 k_{65,1} i_{1}}\right)+q \operatorname{Re}\left(\xi_{1}^{3 k_{51,1} i_{1}}\right)+$ $(2 q-1) \operatorname{Re}\left(\xi_{1}^{k_{51,1} i_{1}}\right)$ for all $i_{1}=1, \ldots, \frac{q-1}{2}$. This implies that either $\xi_{1}^{2 k_{65,1}}=$ 1 , or $\xi_{1}^{k_{51,1}}=1$, or $\xi_{1}^{3 k_{51,1}}=1$. In the first two cases we get a contradiction with the conditions on parameters. So $\xi_{1}^{3 k_{51,1}}=1$, i.e. $\xi_{1}^{k_{51,1}}=\omega^{ \pm 1}$ and $\operatorname{Re}\left(\xi_{1}^{k_{51,1}}\right)=-\frac{1}{2}$. Furthermore, $\operatorname{Re}\left(\xi_{1}^{k_{41,1}}\right)=\operatorname{Re}\left(\xi_{1}^{k_{51,1}}\right)$ and

$$
\operatorname{Re}\left(\xi_{1}^{2 k_{86,1}+k_{86,2}}\right)+\operatorname{Re}\left(\xi_{1}^{2 k_{86,1}-k_{86,2}}\right)=\operatorname{Re}\left(\xi_{1}^{2 k_{65,1}}\right)+\operatorname{Re}\left(\xi_{1}^{k_{41,1}}\right) .
$$

Using the classes $C_{32}\left(i_{1}, q-1\right)$ we have $\operatorname{Re}\left(\xi_{1}^{i_{1} k_{87,1}}\right)+\operatorname{Re}\left(\xi_{1}^{i_{1} k_{87,2}}\right)=$ $\operatorname{Re}\left(\xi_{1}^{i_{1} k_{86,1}}\right)+\operatorname{Re}\left(\xi_{1}^{i_{1} k_{65,1}}\right)=2 \operatorname{Re}\left(\xi_{1}^{i_{1} k_{65,1}}\right)$, for all $i_{1}=1, \ldots, \frac{q-1}{2}$. This implies $\operatorname{Re}\left(\xi_{1}^{k_{87,1}}\right)=\operatorname{Re}\left(\xi^{k_{87,2}}\right)=\operatorname{Re}\left(\xi_{1}^{k_{65,1}}\right)$. Using the classes $C_{68}\left(i_{1}, j, q-\right.$ 
1) we have $\operatorname{Re}\left(\xi_{1}^{k_{87,2}}\right)=\operatorname{Re}\left(\xi_{1}^{k_{41,1}}\right)$. Therefore, $\operatorname{Re}\left(\xi_{1}^{k_{86,1}}\right)=\operatorname{Re}\left(\xi_{1}^{k_{86,2}}\right)=$ $\operatorname{Re}\left(\xi_{1}^{k_{65,1}}\right)+\operatorname{Re}\left(\xi_{1}^{k_{41,1}}\right)=\operatorname{Re}\left(\xi_{1}^{k_{51,1}}\right)=-\frac{1}{2}$. Now, equation (9.1) becomes

$$
\operatorname{Re}\left(\xi^{2 k_{86,1}+k_{86,2}}\right)+\operatorname{Re}\left(\xi^{2 k_{86,1}-k_{86,2}}\right)=-1,
$$

which has no solution with our values of the parameters.

(6) Take $\psi_{84}, \psi_{85}$. Using the classes $C_{74}\left(1, i_{2}, 0\right)$, we have $k_{41,1}=q+1$, a contradiction.

(7) Take $\psi_{86}, \psi_{87}$. Using the classes $C_{72}\left(i_{1}, 1,0\right)$, we have $k_{41,1}=q+1$, a contradiction.

(8) Take $\psi_{92}, \psi_{93}, \psi_{100}$. Using the class $C_{70}(1,0)$, we have $k_{65,1}=q+1$, a contradiction.

(9) Take $\psi_{96}, \psi_{97}$. Using the classes $C_{68}\left(i_{1}, q, 0\right)$, we have $k_{65,1}=q+1$, a contradiction.

(10) Take $\psi_{78}$. Using the classes $C_{70}(q, 0)$ and $C_{72}\left(i_{1}, 0,0\right)$ we obtain $\operatorname{Re}\left(\xi_{1}^{k_{11,1}}\right)=$ $\operatorname{Re}\left(\xi_{1}^{k_{50,1}}\right)=\operatorname{Re}\left(\xi_{1}^{k_{65,1}}\right)$. Using the classes $C_{68}\left(i_{1}, q, 0\right)$ we obtain $\operatorname{Re}\left(\xi_{1}^{k_{87,1}}\right)=$ $\operatorname{Re}\left(\xi_{1}^{k 87,2}\right)$, a contradiction.

(11) Take $\psi_{79}$. Proceed as for $\psi_{78}$.

(12) Take $\psi_{89}$. Using the classes $C_{37}\left(i_{1}, 0\right)$ and $C_{74}\left(i_{1}, i_{2}, 0\right)\left(i_{1}=0,1\right)$, we obtain $1+\operatorname{Re}\left(\xi_{1}^{k_{52,1}}\right)=\operatorname{Re}\left(\xi_{1}^{k_{41,1}}\right)+\operatorname{Re}\left(\xi_{1}^{2 k_{65,1}}\right)$. So either $q+1$ divides $k_{41,1}$ or $(q+1) / 2$ divides $k_{65,1}$. In both the cases, we have a contradiction.

Since all the conjugacy classes that we have considered belong to $H^{\prime}$, it follows from Proposition 6.2 that also $G=H^{\prime}$ has no $p$-vanishing character of degree $|G|_{p}$.

\section{0. $p$-VANISHING CHARACTERS IN HIGH RANK GROUPS}

We can now prove that a Chevalley group of rank $\ell \geq 6$ has no $p$-vanishing character of degree $|G|_{p}$.

Theorem 10.1. Let $G$ be a Chevalley group, and $\chi$ be a p-vanishing character of $G$ of degree $|G|_{p}$. Then $\chi=S t$ in each of the following cases:

(1) $S L(n, q)$ with $n \geq 6$;

(2) $S U(n, q)$ with $n \geq 4$;

(3) $S p(2 m, q)$ with either $m \geq 3$ if $7 \mid(q+1)$ or $m \geq 2$ otherwise;

(4) $\operatorname{Spin}(2 m+1, q)$, q odd, with $m \geq 6$;

(5) $\operatorname{Spin}^{+}(2 m, q)$ with $m \geq 6$;

(6) $\operatorname{Spin}^{-}(2 m, q)$ with $m \geq 3$;

(7) $E_{6}(q), E_{7}(q), E_{8}(q)$;

(8) ${ }^{2} E_{6}(q)$;

(9) $F_{4}(q)$;

(10) $\operatorname{Spin}(2 m+1, q), q$ odd, with either $(q+1,7)=1$ and $m=3,4,5$ or $(q+$ $1,3)=1$ and $m=4,5$.

(11) $\operatorname{Spin}^{+}(2 m, q)$ with $m=4,5$ and $(q+1,3)=1$.

Proof. We proceed by induction on the BN-pair rank $\ell$ of the group. Let $\chi$ be a $p$ vanishing character of $G$ of degree $|G|_{p}$. Take two subsets $J, K$ of $I_{\ell}$ with $I_{\ell}=J \cup K$ and $J \cap K \neq \emptyset$, such that $|K|=2$. By Theorem 8.6. either $\chi=S t$ or $\left(\chi, 1_{G}\right)=1$. In the latter case, $\left(\bar{\chi}_{L}, 1_{L}\right)=1$ for every Levi subgroup $L$ of $G$. So, we obtain the statement if we are able to find $J$ such that $G_{J}$ has no reducible $p$-vanishing characters of degree $\left|G_{J}\right|_{p}$. 
(1) Let $G=S L(n, q)$ with $n \geq 7$. Then we can take $J, K$ as described above, such that $G_{J} \cong S L(n-1, q)$ and $G_{K} \cong S L(3, q)$.

(2) Let $G=S U(n, q)$ with $n \geq 6$. In this case, we can take $J, K$ such that $G_{J} \cong S U(n-2, q)$ and $G_{K} \cong S L(3, q)$.

(3) Let $G=S p(2 m, q)$, with either $m \geq 4$ if 7 divides $q+1$ or $m \geq 3$, otherwise. In this case, we can take $J, K$ such that $G_{J}=S p(2 m-2, q)$ and $G_{K} \cong S L(3, q)$.

(4) Let $G=\operatorname{Spin}(2 m+1, q)$ with $m \geq 6$. In this case, we can take $J, K$ such that $G_{J}=S L(m, q)$ and $G_{K} \cong S p(4, q)$. So the result follows from item (1).

(5) Let $G=\operatorname{Spin}^{+}(2 m, q)$ with $m \geq 6$. In this case, we can take $J, K$ such that $G_{J}=S L(m, q)$ and $G_{K} \cong S L(3, q)$. So the result follows from item (1).

(6) If $G=\operatorname{Spin}^{-}(6, q)$, then $G \cong S U(4, q)$ and so $\chi=S t$ by Lemma 7.6. So, assume that $G=\operatorname{Spin}^{-}(2 m, q)$ with $m>3$. Thus, we can take $J, K$ such that $G_{J} \cong \operatorname{Spin}^{-}(2 m-2, q)$ and $G_{K} \cong S L(3, q)$.

(7) Let $G=E_{n}(q)$ with $n=6,7,8$. In this case we can take $J, K$ such that $G_{J} \cong S L(n, q)$ and $G_{K} \cong S L(3, q)$. So, the result follows from item (1).

(8) Let $G={ }^{2} E_{6}(q)$. In this case we can take $J, K$ such that $G_{J} \cong S U(6, q)$ and $G_{K} \cong S L(3, q)$.

(9) Let $G=F_{4}(q)$. In this case we can take $J, K$ such that $G_{J} \cong S p(6, q)$ and $G_{K} \cong \Omega(5, q)$.

(10) Let $G=\operatorname{Spin}(2 m+1, q)$ and $q$ odd. Assume first that $(q+1,7)=1$. In this case, for $m=3,4,5$ we can take $G_{J} \cong \operatorname{Spin}(2 m-1, q)$ and $G_{K} \cong S L(3, q)$. Now, suppose that $(q+1,3)=1$. Hence, for $m=4,5$ we can take $G_{J} \cong S L(m, q)$ and $G_{K} \cong S p(4, q)$.

(11) Let $G=\operatorname{Spin}^{+}(2 m, q)$ with $m=4,5$ and $(q+1,3)=1$. In this case we can take $J, K$ such that $G_{J} \cong S L(m, q)$ and $G_{K} \cong S L(3, q)$. So the result follows from Lemmas 9.1 and 9.2 . 


\section{The TABLES}

The tables below contain information on the $S y l_{p}$-vanishing characters and, when they exist, on the reducible $p$-vanishing characters of degree $S t(1)$. (This equals the order of a Sylow $p$-subgroup $U$ of the group in question.) The tables are organized as follows. For every quasi-simple Chevalley group $G$ and/or its version $H$ with connected center we assign a subsection in which we provide some properties of the groups $G, H$ and their characters, necessary for understanding our results about these groups tabulated in the same subsection. In some cases we have distinct subsections for the same group $H$, depending on whether $q$ is odd or even, or congruent to 1 or -1 modulo 3 , etc.

First we recall that CHEVIE partitions the conjugacy classes and irreducible characters of $H$ in subsets which we denote here by $\mathbf{C}_{h}$ and $\mathbf{X}_{h}(h=1,2, \ldots)$. Note that the size of every class in $\mathbf{C}_{h}$ is the same. Similarly, the degrees of the characters in $\mathbf{X}_{h}$ are the same. (Sometimes the degrees of characters from distinct $\mathbf{X}_{h}$ may coincide.) We keep the notation of CHEVIE for irreducible characters and conjugacy classes. The symbol $\chi_{h}(k)$ identifies the irreducible character of the set $\mathbf{X}_{h}$ associated to the particular element $k \in I_{h}$ according to CHEVIE. We denote by $I_{h}$ the set of parameters describing the individual elements of $\mathbf{X}_{h}$. If it is not necessary to specify the parameter $k$, we simply write $\chi_{h}$ to indicate any element of the set $\mathbf{X}_{h}$. Similarly, an individual conjugacy class $C_{h}(k) \in \mathbf{C}_{h}$ is the class which corresponds to the parameter $k \in I_{h}$.

The parameters $k \in I_{h}$ labeling individual characters $\chi_{h}(k) \in \mathbf{X}_{h}$ and classes $C_{h}(k) \in \mathbf{C}_{h}$ are represented as elements of a direct product of cyclic groups, with some conditions (note that not necessarily to distinct parameters correspond distinct characters). In the tables we indicate the group $I_{h}$ belongs to in the columns headed $I_{h}$ and the exact description of $I_{h}$ is obtained by the exclusion of the elements written in the columns headed 'exceptions'.

In each subsection we usually give two or three tables headed as Table A, Table B, Table C and Table D (in some cases Table D is omitted).

Tables A provides some information on the irreducible characters of $H$ occurred in Tables B,C,D. Specifically, Tables A list the degrees of the members of the sets $\mathbf{X}_{h}$, the range of $h$ and we also describe the structure of the character parameter group $I_{h}$. In some degenerate case the set $\mathbf{X}_{h}$ consists of a single element, so no parameter needs to be assigned to the element of the corresponding set $I_{h}$. In this case the respective position in the table is marked by - , see for instance Table 11.2 2.A. In order to avoid misunderstanding with CHEvIE notation $\chi_{h}(k)$, in Tables A we use $\operatorname{deg} \chi_{h}$ to indicate the degree of a character $\chi_{h}$.

In Tables B, we report the $S y l_{p}$-decompositions that we have found and used in our computations, see Sections [5, 7 and 9.

In Tables C we list all the $S y l_{p}$-vanishing characters of degree $|H|_{p}$. The column headed by $\mathbf{v}$ lists, in more friendly notation, the $S y l_{p}$-vanishing characters $\psi=$ $\sum \chi_{h}$, where $\chi_{h} \in \mathbf{X}_{h}$ and the sum ranges over the indices from $\mathbf{v}$. Say, if $\mathbf{v}=(1,4)$ then $\psi=\chi_{1}+\chi_{4}$. The column headed by $j$ is simply the ordering numbers for $\mathbf{v}$; we could write $\mathbf{v}_{j}$ to be more precise.

Finally, in Tables D, when they exist, we provide the values of the $p$-vanishing characters $\psi$ of degree $|H|_{p}$ satisfying the condition $\left(\psi, 1_{H}\right)=1$. Note that no information on the character values is lost because all the other $p$-vanishing characters can be obtained from these by multiplying them with linear characters of 
$H$. In addition, we explicitly write down only the values of $\psi$ at those conjugacy classes where $\psi$ differs from the Steinberg character. Unless otherwise stated, the conjugacy classes in Table D are parametrised by the same sets $I_{h}$ that are used to parametrise the characters in Table A.

To facilitate understanding the tables, as an illustration, in Section 11.1 for groups $G L(2, q)$ and $S L(2, q)$ we write additional comments about the table content.

We use the following notation for some primitive complex roots of the unity: $\omega=\exp \left(\frac{2 \pi i}{3}\right), \xi_{1}=\exp \left(\frac{2 \pi i}{q+1}\right), \xi_{2}=\exp \left(\frac{2 \pi i}{q^{2}+1}\right), \varphi_{8}^{\prime}=\exp \left(\frac{2 \pi i}{q^{2}+\sqrt{2} q+1}\right)$ and $\varphi_{12}^{\prime \prime}=$ $\exp \left(\frac{2 \pi i}{q^{2}+\sqrt{3} q+1}\right)$.

\subsection{Groups $H=G L(2, q)$.}

Table (11.1 A). ChEviE sets $\mathbf{X}_{h}$, their degrees and parameters for $G L(2, q)$.

\begin{tabular}{|c|c|c|c|c|c|c|}
\hline & $\operatorname{der} \gamma$ & & & $h$ & $I_{h}$ & exceptions \\
\hline 1 & 1 & $\frac{\pi}{2}$ & ueg. & 1,2 & $k \in \mathbb{Z}_{q-1}$ & \\
\hline 3 & $q+1$ & 4 & $q-1$ & $\begin{array}{l}3 \\
4\end{array}$ & $\begin{array}{l}(k, l) \in \mathbb{Z}_{q-1}^{2} \\
k \in \mathbb{Z}_{\alpha^{2}-1}\end{array}$ & $\begin{array}{l}(q-1) \mid(k-l) \\
(q+1) \mid k\end{array}$ \\
\hline
\end{tabular}

These two tables provide the following information. The irreducible characters of the group $G L(2, q)$ are partitioned into 4 sets $\mathbf{X}_{h}$. The characters of the set $\mathbf{X}_{1}$ have degree $\operatorname{deg} \chi_{1}=1$ and are parametrised by the elements of the set $I_{1}=\mathbb{Z}_{q-1}$. This means that $\mathbf{X}_{1}=\left\{\chi_{1}(0), \ldots, \chi_{1}(q-2)\right\}$. The characters of the set $\mathbf{X}_{2}$ have degree $q$ and are parametrised by the elements of $I_{2}=\mathbb{Z}_{q-1}$. So, $\mathbf{X}_{2}=\left\{\chi_{2}(u) \mid u \in \mathbb{Z}_{q-1}\right\}$. The characters of the set $\mathbf{X}_{3}$ have degree $q+1$ and are parametrised by the elements of $I_{3}=\mathbb{Z}_{q-1} \times \mathbb{Z}_{q-1}$. One more time, we put in evidence that different choices of the parameters $(k, l) \in I_{3}$ may identify the same irreducible character. Furthermore, the exceptions listed in the third column mean that the character $\chi_{3}(k, l)$ is not a member of $\mathbf{X}_{3}$ whenever $(q-1) \mid(k-l)$. It turns out that the set $\mathbf{X}_{3}$ contains $(q-2)(q-1) / 2$ distinct characters. Finally, the characters of the set $\mathbf{X}_{4}$ have degree $q-1$ and are parametrised by the elements of $I_{4}=\mathbb{Z}_{q^{2}-1}$. In this case, $\chi_{4}(k)$ does not belong to $\mathbf{X}_{4}$, whenever $(q+1) \mid k$. (Observe that $(q-1) \mid(k-l)$ is equivalent to $k=l$, but we prefer to keep CHEviE notation.)

Table (11.1łB). Some $S y l_{p}$-decompositions of irreducible characters for $G L(2, q)$.

$$
\begin{array}{c|c||c|c}
h & \mathbf{v} & h & \mathbf{v} \\
\hline 2 & (1,4) & 3 & (1,2)
\end{array}
$$

This table give the following information on the $S y l_{p}$-decompositions that we were able to find. The restriction to $U$ of each character $\chi_{2}$ belonging to the set $\mathbf{X}_{2}$ can be written as the sum of two summands: the first one is the restriction of any character of the set $\mathbf{X}_{1}$, the second one is the restriction of any character of the set $\mathbf{X}_{4}$. Briefly, using the notation of Section $4, \chi_{2} \equiv \chi_{1}+\chi_{4}(\bmod U)$. Similarly, $\chi_{3} \equiv \chi_{1}+\chi_{2}(\bmod U)$.

Table (11.17C). The $S y l_{p}$-vanishing characters of degree $\operatorname{St}(1)$ for $G L(2, q)$.

\begin{tabular}{c|c||c|c}
$j$ & $\mathbf{v}$ & $j$ & $\mathbf{v}$ \\
\hline 1 & $(1,4)$ & 2 & $(2)$
\end{tabular}

This table lists all $S y l_{p}$-characters $\psi_{j}$ of $H=G L(2, q)$ of degree $|H|_{p}$. For $H$ they are of two types: $\psi_{1}=\chi_{1}+\chi_{4}$ (i.e. the sum of two irreducible characters: the first one is any character belonging to the set $\mathbf{X}_{1}$, the second one is any character belonging to the set $\mathbf{X}_{4}$ ); $\psi_{2}=\chi_{2}$ (i.e. any character of the set $\mathbf{X}_{2}$ ). 
Table (11.1 D). The $p$-vanishing characters of degree $S t(1)$ and their values for $G L(2, q)$.

In view of Lemma 7.12 we have the character $\psi=1_{H}+\chi_{4}(k)$, where $k \in I_{4}$ and $k=(q-1) k^{\prime}$. This character differs from $S t$ only on the following classes:

$$
\begin{array}{l||l|l}
C_{h} & S t & \psi \\
\hline C_{4}(i) & -1 & 1-2 \operatorname{Re}\left(\xi_{1}^{i k^{\prime}}\right)
\end{array}
$$

In the above table, we give the values of the reducible $p$-vanishing character $\psi$ and those of the Steinberg character $S t$ of $H$ only at the conjugacy classes where these values differ. The notation for the conjugacy classes, which is similar to the notation for the irreducible characters, is taken according to CHEviE.

11.2. Groups $U(3, q)$ and $S U(3, q)$.

Table (11.21-A). CHEviE sets $\mathbf{X}_{h}$, their degrees and parameters for $U(3, q)$.

\begin{tabular}{l|l||l|l||l|l}
$h$ & $\operatorname{deg} \chi_{h}$ & $h$ & $\operatorname{deg} \chi_{h}$ & $h$ & $\operatorname{deg} \chi_{h}$ \\
\hline 1 & 1 & 2 & $q(q-1)$ & 3 & $q^{3}$ \\
4 & $q^{2}-q+1$ & 5 & $q\left(q^{2}-q+1\right)$ & 6 & $(q-1)\left(q^{2}-q+1\right)$ \\
7 & $(q+1)\left(q^{2}-q+1\right)$ & 8 & $(q+1)^{2}(q-1)$ & \\
$h$ & $I_{h}$ & exceptions \\
\hline $1,2,3$ & $u \in \mathbb{Z}_{q+1}$ & $(q+1) \mid(u-v)$ \\
4,5 & $(u, v) \in \mathbb{Z}_{q+1} \times \mathbb{Z}_{q+1}$ & $(q+1) \mid(u-v),(u-w),(v-w)$ \\
6 & $(u, v, w) \in \mathbb{Z}_{q+1} \times \mathbb{Z}_{q+1} \times \mathbb{Z}_{q+1}$ & $(q-1) \mid u$ \\
7 & $(v, u) \in \mathbb{Z}_{q+1} \times \mathbb{Z}_{q^{2}-1}$ & $\left(q^{2}-q+1\right) \mid u$
\end{tabular}

Table (11.21-B). Some $S y l_{p}$-decompositions of irreducible characters for $U(3, q)$.

\begin{tabular}{c|c||c|c||c|c||c|c||c|c||c|c}
$h$ & $\mathbf{v}$ & $h$ & $\mathbf{v}$ & $h$ & $\mathbf{v}$ & $h$ & $\mathbf{v}$ & $h$ & $\mathbf{v}$ & $h$ & $\mathbf{v}$ \\
\hline 3 & $(2,5)$ & 4 & $(1,2)$ & 5 & $(4,6)$ & 7 & $(1,3)$ & 7 & $(4,5)$ & 8 & $(2,2,2,6)$
\end{tabular}

The above table describes 6 types of $S y l_{p}$-decompositions of irreducible characters of $U(3, q)$. For instance, the last column tells us that we have $\chi_{8} \equiv \chi_{2}+\chi_{2}^{\prime}+$ $\chi_{2}^{\prime \prime}+\chi_{6}(\bmod U)$. This means that the restriction to $U$ of any character of the set $\mathbf{X}_{8}$ is the sum of the restrictions of four characters: any 3 characters $\chi_{2}, \chi_{2}^{\prime}, \chi_{2}^{\prime \prime}$ of the set $\mathbf{X}_{2}$ (distinct or not) and an arbitrary character $\chi_{6}$ from $\mathbf{X}_{6}$.

Table (11.21-C). The $S y l_{p}$-vanishing characters of degree $\operatorname{St}(1)$ for $U(3, q)$.

$$
\begin{array}{c|c||c|c||c|c||c|c}
j & \mathbf{v} & j & \mathbf{v} & j & \mathbf{v} & j & \mathbf{v} \\
\hline 1 & (1,2,2,6) & 2 & (2,4,6) & 3 & (2,5) & 4 & (3)
\end{array}
$$

Note that the above table gives the $S y l_{p}$-vanishing character $\psi_{1}=\chi_{1}+\chi_{2}+$ $\chi_{2}^{\prime}+\chi_{6}$. This means that $\psi_{1}$ is the sum of 4 characters: $\chi_{1}$ is any character the set $\mathbf{X}_{1}, \chi_{2}, \chi_{2}^{\prime}$ are any characters (distinct or not) of $\mathbf{X}_{2}$, and $\chi_{6}$ is any character of $\mathbf{X}_{6}$.

Table (11.2 1-D). The $p$-vanishing characters of degree $S t(1)$ and their values for $U(3, q)$ when 3 divides $q+1$.

In view of Lemma 5.4, we have the character $\psi=1_{H}+\chi_{2}(a)+\chi_{2}(2 a)+$ $\chi_{6}(a, 2 a, 3 a)$, where $a=(q+1) / 3$. This character differs from $S t$ only on the following classes:

$$
\begin{array}{l||l|l}
C_{h} & S t & \psi \\
\hline C_{6}(k, l, m) & -1 & 1+4 \operatorname{Re}\left(\omega^{k+l+m}\right)-2 \operatorname{Re}\left(\omega^{k-l}\right)-2 \operatorname{Re}\left(\omega^{k-m}\right)-2 \operatorname{Re}\left(\omega^{l-m}\right) \\
C_{8}(k) & -1 & 1-2 \operatorname{Re}\left(\omega^{k}\right)
\end{array}
$$


Table (11.2-2.A). CHEvIE sets $\mathbf{X}_{h}$, their degrees and parameters for $S U(3, q)$ with the condition that $3 \nmid(q+1)$.

\begin{tabular}{|c|c|c|c|c|c|c|}
\hline$h$ & $\operatorname{deg} \chi_{h}$ & \begin{tabular}{l|l}
$h$ & $\mathrm{deg}$ \\
\end{tabular} & & $\mid h$ & $\operatorname{deg} \chi_{h}$ & \begin{tabular}{l|l}
$h$ & $\operatorname{deg} \chi_{h}$ \\
\end{tabular} \\
\hline 1 & & \begin{tabular}{l|l}
2 & $q(c$ \\
\end{tabular} & -1) & $\mid$\begin{tabular}{||l|}
3 \\
3
\end{tabular} & & \begin{tabular}{l|l}
4 & $q^{2}-q+1$
\end{tabular} \\
\hline 5 & $q\left(q^{2}-q+1\right)$ & $(q$ & -1) $\left(q^{2}-q+1\right)$ & 7 & $(q+1)\left(q^{2}-q+1\right)$ & $8 \mid(q+1)^{2}(q-1)$ \\
\hline & & $h$ & $I_{h}$ & & exceptions & \\
\hline & & $1,2,3$ & - & & & \\
\hline & & 4,5 & $n \in \mathbb{Z}_{q+1}$ & & $(q+1) \mid n$ & \\
\hline & & 6 & $(n, m) \in \mathbb{Z}_{q+1}$ & $\times \mathbb{Z}_{q+1}$ & $(q+1) \mid(n-m)$ & \\
\hline & & 7 & $n \in \mathbb{Z}_{q^{2}-1}$ & & $(q-1) \mid n$ & \\
\hline & & 8 & $n \in \mathbb{Z}_{q^{2}-q+1}$ & & $\left(q^{2}-q+1\right) \mid n$ & \\
\hline
\end{tabular}

Note that each set $\mathbf{X}_{1}, \mathbf{X}_{2}$ and $\mathbf{X}_{3}$ consists of a single character.

Table (11.2 2-B). Some $S y l_{p}$-decompositions of irreducible characters for $S U(3, q)$ with the condition that $3 \nmid(q+1)$.

\begin{tabular}{c|c||c|c||c|c||c|c||c|c||c|c}
$h$ & $\mathbf{v}$ & $h$ & $\mathbf{v}$ & $h$ & $\mathbf{v}$ & $h$ & $\mathbf{v}$ & $h$ & $\mathbf{v}$ & $h$ & $\mathbf{v}$ \\
\hline 3 & $(2,5)$ & 4 & $(1,2)$ & 5 & $(4,6)$ & 7 & $(1,3)$ & 7 & $(4,5)$ & 8 & $(2,2,2,6)$
\end{tabular}

Table $[11.2,2-\mathrm{C})$. The $S y l_{p}$-vanishing characters of degree $\operatorname{St}(1)$ for $S U(3, q)$ with the condition that $3 \nmid(q+1)$.

\begin{tabular}{c|c||c|c||c|c||c|c}
$j$ & $\mathbf{v}$ & $j$ & $\mathbf{v}$ & $j$ & $\mathbf{v}$ & $j$ & $\mathbf{v}$ \\
\hline 1 & $(1,2,2,6)$ & 2 & $(2,4,6)$ & 3 & $(2,5)$ & 4 & $(3)$
\end{tabular}

Table (11.2 3-A). CHEvIE sets $\mathbf{X}_{h}$, their degrees and parameters for $S U(3, q)$ with the condition that $3 \mid(q+1)$.

\begin{tabular}{l|l||l|l||l|l}
$h$ & $\operatorname{deg} \chi_{h}$ & $h$ & $\operatorname{deg} \chi_{h}$ & $h$ & $\operatorname{deg} \chi_{h}$ \\
\hline 1 & 1 & 2 & $q(q-1)$ & 3 & $q^{3}$ \\
4 & $q^{2}-q+1$ & 5 & $q\left(q^{2}-q+1\right)$ & 6 & $(q+1)\left(q^{2}-q+1\right)$ \\
$7,8,9$ & $\frac{1}{3}(q-1)\left(q^{2}-q+1\right)$ & 10 & $(q-1)\left(q^{2}-q+1\right)$ & $11, \ldots, 16$ & $\frac{1}{3}(q+1)^{2}(q-1)$ \\
17 & $(q+1)^{2}(q-1)$ & & \\
& $h$ & $I_{h}$ & exceptions \\
\cline { 2 - 4 } & $1,2,3,7,8,9,11, \ldots, 16$ & - & \\
& 4,5 & $n \in \mathbb{Z}_{q+1}$ & $(q+1) \mid n$ \\
& 6 & $n \in \mathbb{Z}_{q^{2}-1}$ & $(q-1) \mid n$ \\
& 10 & $(n, m) \in \mathbb{Z}_{q+1} \times \mathbb{Z}_{q+1}$ & $(q+1) \mid n, m,(n-m)$ \\
& 17 & $n \in \mathbb{Z}_{q^{2}-q+1}$ & $\frac{q^{2}-q+1}{3} \mid n$
\end{tabular}

Table 11.23 -B). Some $S y l_{p}$-decompositions of irreducible characters for $S U(3, q)$ with the condition that $3 \mid(q+1)$.

\begin{tabular}{c|c||c|c||c|c||c|c||c|c}
$h$ & $\mathbf{v}$ & $h$ & $\mathbf{v}$ & $h$ & $\mathbf{v}$ & $h$ & $\mathbf{v}$ & $h$ & $\mathbf{v}$ \\
\hline 11 & $(14)$ & 12 & $(16)$ & 13 & $(15)$ & 3 & $(2,5)$ & 4 & $(1,2)$ \\
5 & $(4,10)$ & 6 & $(1,3)$ & 6 & $(4,5)$ & 14 & $(2,7)$ & 15 & $(2,9)$ \\
16 & $(2,8)$ & 10 & $(7,8,9)$ & 17 & $(2,2,2,7,8,9)$ & & & &
\end{tabular}

Note that the characters of the set $\mathbf{X}_{11}$ are $S y l_{p}$-equivalent to those of $\mathbf{X}_{14}$. Similarly for the pairs $\left(\mathbf{X}_{12}, \mathbf{X}_{16}\right)$ and $\left(\mathbf{X}_{13}, \mathbf{X}_{15}\right)$. 
Table (11.2,3-C). The $S y l_{p}$-vanishing characters of degree $S t(1)$ for $S U(3, q)$ with the condition that $3 \mid(q+1)$.

\begin{tabular}{c|c||c|c||c|c||c|c}
$j$ & $\mathbf{v}$ & $j$ & $\mathbf{v}$ & $j$ & $\mathbf{v}$ & $j$ & $\mathbf{v}$ \\
\hline 1 & $(1,2,2,7,8,9)$ & 2 & $(1,2,2,10)$ & 3 & $(1,2,7,8,13)$ & 4 & $(1,2,7,8,15)$ \\
5 & $(1,2,7,9,12)$ & 6 & $(1,2,7,9,16)$ & 7 & $(1,2,8,9,11)$ & 8 & $(1,2,8,9,14)$ \\
9 & $(1,7,12,13)$ & 10 & $(1,7,12,15)$ & 11 & $(1,7,13,16)$ & 12 & $(1,7,15,16)$ \\
13 & $(1,8,11,13)$ & 14 & $(1,8,11,15)$ & 15 & $(1,8,13,14)$ & 16 & $(1,8,14,15)$ \\
17 & $(1,9,11,12)$ & 18 & $(1,9,11,16)$ & 19 & $(1,9,12,14)$ & 20 & $(1,9,14,16)$ \\
21 & $(2,4,7,8,9)$ & 22 & $(2,4,10)$ & 23 & $(2,5)$ & 24 & $(3)$ \\
25 & $(4,7,8,13)$ & 26 & $(4,7,8,15)$ & 27 & $(4,7,9,12)$ & 28 & $(4,7,9,16)$ \\
29 & $(4,8,9,11)$ & 30 & $(4,8,9,14)$ & & & &
\end{tabular}

Table 11.2,3-D). The $p$-vanishing characters of degree $S t(1)$ and their values for $S U(3, q)$ with the condition that $3 \mid(q+1)$.

In view of Lemma 5.6, we have the character $\psi=1_{G}+2 \chi_{2}+\chi_{7}+\chi_{8}+\chi_{9}$. This character differs from $S t$ only on the following classes:

$$
\begin{array}{l||l|l}
C_{h} & S t & \psi \\
\hline C_{8}(a, b) & -1 & 5-6 \operatorname{Re}\left(\omega^{a-b}\right)
\end{array}
$$

Here $(a, b) \in I_{8}=\mathbb{Z}_{q+1} \times \mathbb{Z}_{q+1}$ with the exceptions: $(q+1) \mid a, b,(a-b)$.

11.3. Groups ${ }^{2} B_{2}\left(q^{2}\right)$.

Table (11.3 A). CHEviE sets $\mathbf{X}_{h}$, their degrees and parameters for ${ }^{2} B_{2}\left(q^{2}\right)$.

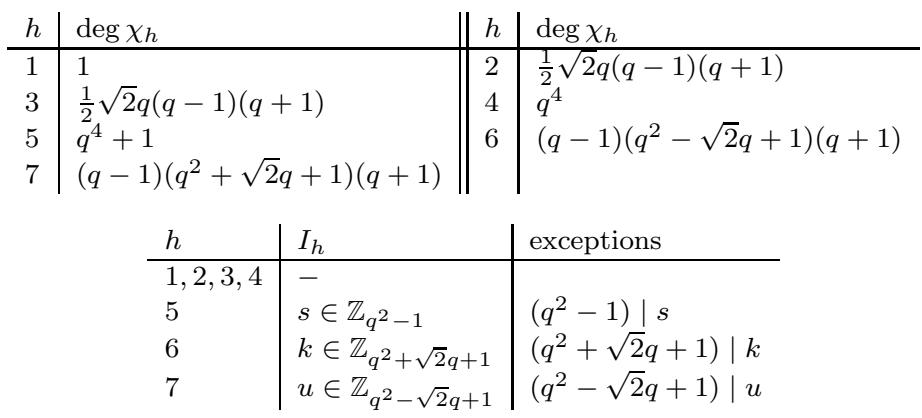

Table (11.3+B). Some $S y l_{p}$-decompositions of irreducible characters for ${ }^{2} B_{2}\left(q^{2}\right)$.

\begin{tabular}{c|c||c|c||c|c}
$h$ & $\mathbf{v}$ & $h$ & $\mathbf{v}$ & $h$ & $\mathbf{v}$ \\
\hline 5 & $(1,4)$ & 4 & $(1,2,3,6)$ & 7 & $(2,2,3,3,6)$
\end{tabular}

Table (11.3 $\mathrm{C})$. The $S y l_{p}$-vanishing characters of degree $S t(1)$ for ${ }^{2} B_{2}\left(q^{2}\right)$.

$$
\begin{array}{c|c||c|c}
j & \mathbf{v} & j & \mathbf{v} \\
\hline 1 & (1,2,3,6) & 2 & (4)
\end{array}
$$

Table (11.3-D). The $p$-vanishing characters of degree $S t(1)$ and their values for ${ }^{2} B_{2}\left(q^{2}\right)$.

In view of Lemma 5.7 we have the character $\psi=1_{G}+\chi_{2}+\chi_{3}+\chi_{6}(k)$. This character differs from $S t$ only on the following classes:

$$
\begin{array}{l||l|l}
C_{h} & S t & \psi \\
\hline C_{6}(b) & -1 & 3-2 \operatorname{Re}\left(\left(\varphi_{8}^{\prime}\right)^{b k}\right)-2 \operatorname{Re}\left(\left(\varphi_{8}^{\prime}\right)^{q^{2} b k}\right)
\end{array}
$$


11.4. Groups ${ }^{2} G_{2}\left(q^{2}\right)$.

Table (11.4 A). CHEviE sets $\mathbf{X}_{h}$, their degrees and parameters for ${ }^{2} G_{2}\left(q^{2}\right)$.

\begin{tabular}{l|l||l|l}
$h$ & $\operatorname{deg} \chi_{h}$ & $h$ & $\operatorname{deg} \chi_{h}$ \\
\hline 1 & 1 & 2 & $\frac{1}{6} \sqrt{3} q\left(q^{2}-1\right)\left(q^{2}+\sqrt{3} q+1\right)$ \\
3 & $\frac{1}{6} \sqrt{3} q\left(q^{2}-1\right)\left(q^{2}-\sqrt{3} q+1\right)$ & 4 & $\frac{1}{6} \sqrt{3} q\left(q^{2}-1\right)\left(q^{2}+\sqrt{3} q+1\right)$ \\
5 & $\frac{1}{6} \sqrt{3} q\left(q^{2}-1\right)\left(q^{2}-\sqrt{3} q+1\right)$ & 6 & $\frac{1}{3} \sqrt{3} q(q-1)\left(q^{2}+1\right)(q+1)$ \\
7 & $\frac{1}{3} \sqrt{3} q(q-1)\left(q^{2}+1\right)(q+1)$ & 8 & $q^{6}$ \\
9 & $q^{4}-q^{2}+1$ & 10 & $q^{2}\left(q^{4}-q^{2}+1\right)$ \\
11 & $\left(q^{2}+1\right)\left(q^{4}-q^{2}+1\right)$ & 12 & $\left(q^{2}-1\right)\left(q^{2}+\sqrt{3} q+1\right)\left(q^{2}+1\right)$ \\
13 & $\left(q^{2}-1\right)\left(q^{4}-q^{2}+1\right)$ & 14 & $\left(q^{2}-1\right)\left(q^{2}-\sqrt{3} q+1\right)\left(q^{2}+1\right)$
\end{tabular}

\begin{tabular}{l|l|l}
$h$ & $I_{h}$ & exceptions \\
\hline $1, \ldots, 10$ & - & \\
11 & $k \in \mathbb{Z}_{q^{2}-1}$ & $\frac{q^{2}-1}{2} \mid k$ \\
12 & $k \in \mathbb{Z}_{q^{2}-\sqrt{3} q+1}$ & $\left(q^{2}-\sqrt{3} q+1\right) \mid k$ \\
13 & $(k, l) \in \mathbb{Z}_{\frac{q^{2}+1}{2}} \times \mathbb{Z}_{2}$ & $\frac{q^{2}+1}{4} \mid k$ \\
14 & $k \in \mathbb{Z}_{q^{2}+\sqrt{3} q+1}$ & $\left(q^{2}-\sqrt{3} q+1\right) \mid k$
\end{tabular}

Table (11.4 B). Some decompositions of the restrictions of the irreducible characters on the classes $C_{1}, C_{2}, C_{5}$ for ${ }^{2} G_{2}\left(q^{2}\right)$.

\begin{tabular}{c|c||c|c||c|c||c|c}
$h$ & $\mathbf{v}$ & $h$ & $\mathbf{v}$ & $h$ & $\mathbf{v}$ & $h$ & $\mathbf{v}$ \\
\hline 2 & $(4)$ & 3 & $(5)$ & 6 & $(7)$ & 10 & $(9,13)$ \\
11 & $(1,8)$ & 11 & $(9,10)$ & 8 & $(1,3,5,6,7,14)$ & &
\end{tabular}

Table (11.4.C). The $S y l_{p}$-vanishing characters of degree $S t(1)$ for ${ }^{2} G_{2}\left(q^{2}\right)$.

$$
\begin{array}{c|c||c|c}
j & \mathbf{v} & j & \mathbf{v} \\
\hline 1 & (1,3,5,6,7,14) & 2 & (8)
\end{array}
$$

Table (11.4 D). The $p$-vanishing characters of degree $S t(1)$ and their values for ${ }^{2} G_{2}\left(q^{2}\right)$.

In view of Lemma 5.8 we have the character $\psi=1_{G}+\chi_{3}+\chi_{5}+\chi_{6}+\chi_{7}+\chi_{14}(k)$. This character differs from $S t$ only on the following classes:

$$
\begin{array}{l||l|l}
C_{h} & S t & \psi \\
\hline C_{14}(i) & -1 & 5-2 \operatorname{Re}\left(\left(\varphi_{12}^{\prime \prime}\right)^{i k}\right)-2 \operatorname{Re}\left(\left(\varphi_{12}^{\prime \prime}\right)^{(\sqrt{3} q+1) i k}\right)-2 \operatorname{Re}\left(\left(\varphi_{12}^{\prime \prime}\right)^{(\sqrt{3} q+2) i k}\right)
\end{array}
$$

11.5. Groups $G L(3, q)$ and $S L(3, q)$.

Table (11.51-A). CHEVIE sets $\mathbf{X}_{h}$, their degrees and parameters for $G L(3, q)$.

\begin{tabular}{l|l||c|l}
$h$ & $\operatorname{deg} \chi_{h}$ & $h$ & $\operatorname{deg} \chi_{h}$ \\
\hline 1 & 1 & 2 & $q(q+1)$ \\
3 & $q^{3}$ & 4 & $q^{2}+q+1$ \\
5 & $q\left(q^{2}+q+1\right)$ & 6 & $(q+1)\left(q^{2}+q+1\right)$ \\
7 & $(q-1)\left(q^{2}+q+1\right)$ & 8 & $(q-1)^{2}(q+1)$
\end{tabular}

\begin{tabular}{l|l|l}
$h$ & $I_{h}$ & exceptions \\
\hline $1,2,3$ & $n \in \mathbb{Z}_{q-1}$ & \\
4,5 & $(n, m) \in \mathbb{Z}_{q-1} \times \mathbb{Z}_{q-1}$ & $(q-1) \mid(n-m)$ \\
6 & $(n, m, l) \in \mathbb{Z}_{q-1} \times \mathbb{Z}_{q-1} \times \mathbb{Z}_{q-1}$ & $(q-1) \mid(n-m),(n-l),(m-l)$ \\
7 & $(m, n) \in \mathbb{Z}_{q-1} \times \mathbb{Z}_{q^{2}-1}$ & $(q+1) \mid n$ \\
8 & $n \in \mathbb{Z}_{q^{3}-1}$ & $\left(q^{2}+q+1\right) \mid n$
\end{tabular}

Table (11.5,1-B). Some $S y l_{p}$-decompositions of irreducible characters for $G L(3, q)$.

\begin{tabular}{c|c||c|c||c|c||c|c||c|c}
$h$ & $\mathbf{v}$ & $h$ & $\mathbf{v}$ & $h$ & $\mathbf{v}$ & $h$ & $\mathbf{v}$ & $h$ & $\mathbf{v}$ \\
\hline 3 & $(1,7)$ & 4 & $(1,2)$ & 5 & $(2,3)$ & 5 & $(4,7)$ & 6 & $(4,5)$
\end{tabular}


Table (11.51-C). The $S y l_{p}$-vanishing characters of degree $S t(1)$ for $G L(3, q)$.

$$
\begin{array}{c|c||c|c}
j & \mathbf{v} & j & \mathbf{v} \\
\hline 1 & (1,7) & 2 & (3)
\end{array}
$$

Table (11.5 1-D). The $p$-vanishing characters of degree $S t(1)$ and their values for $G L(3, q)$.

In view of Lemma 7.4 we have the character $\psi=1_{H}+\chi_{7}(q-1, n)$, with $n=$ $(q-1) n^{\prime}$. This character differs from $S t$ only on the following classes:

$$
\begin{array}{l||l|l}
C_{h} & S t & \psi \\
\hline C_{7}(a, b) & -1 & 1-2 \operatorname{Re}\left(\xi_{1}^{b n^{\prime}}\right)
\end{array}
$$

Table (11.5,2-A). CHEVIE sets $\mathbf{X}_{h}$, their degrees and parameters for $S L(3, q)$ with the condition that $3 \mid(q-1)$.

\begin{tabular}{l|l||l|l}
$h$ & $\operatorname{deg} \chi_{h}$ & $h$ & $\operatorname{deg} \chi_{h}$ \\
\hline 1 & 1 & 2 & $q(q+1)$ \\
3 & $q^{3}$ & 4 & $q^{2}+q+1$ \\
5 & $q\left(q^{2}+q+1\right)$ & $6,7,8$ & $\frac{1}{3}(q+1)\left(q^{2}+q+1\right)$ \\
9 & $(q+1)\left(q^{2}+q+1\right)$ & 10 & $(q-1)\left(q^{2}+q+1\right)$ \\
$11, \ldots, 16$ & $\frac{1}{3}(q-1)^{2}(q+1)$ & 17 & $(q-1)^{2}(q+1)$ \\
$h$ & $I_{h}$ & exceptions \\
\hline $1,2,3,6,7,8,11, \ldots, 16$ & - & \\
4,5 & $n \in \mathbb{Z}_{q-1}$ & $(q-1) \mid n$ \\
9 & $(n, m) \in \mathbb{Z}_{q-1}^{2}$ & $(q-1) \mid n, m,(n-m)$ \\
10 & $n \in \mathbb{Z}_{q^{2}-1}$ & $(q+1) \mid n$ \\
17 & $n \in \mathbb{Z}_{q^{2}+q+1}$ & $\frac{q^{2}+q+1}{3} \mid n$
\end{tabular}

Table (11.5,2-B). Some $S y l_{p}$-decompositions of irreducible characters for $S L(3, q)$ with the condition that $3 \mid(q-1)$.

\begin{tabular}{c|c||c|c||c|c||c|c||c|c||c|c}
$h$ & $\mathbf{v}$ & $h$ & $\mathbf{v}$ & $h$ & $\mathbf{v}$ & $h$ & $\mathbf{v}$ & $h$ & $\mathbf{v}$ & $h$ & $\mathbf{v}$ \\
\hline 11 & $(14)$ & 12 & $(16)$ & 13 & $(15)$ & 3 & $(1,10)$ & 4 & $(1,2)$ & 5 & $(2,3)$ \\
5 & $(4,10)$ & 6 & $(2,14)$ & 7 & $(2,16)$ & 8 & $(2,15)$ & 9 & $(4,5)$ & 17 & $(14,15,16)$
\end{tabular}

Table (11.52-C). The $S y l_{p}$-vanishing characters of degree $S t(1)$ for $S L(3, q)$ with the condition that $3 \mid(q-1)$.

\begin{tabular}{c|c||c|c}
$j$ & $\mathbf{v}$ & $j$ & $\mathbf{v}$ \\
\hline 1 & $(1,10)$ & 2 & $(3)$
\end{tabular}

Table (11.5,3-A). CHEVIE sets $\mathbf{X}_{h}$, their degrees and parameters for $S L(3, q)$ with the condition that $3 \nmid(q-1)$.

\begin{tabular}{l|l||l|ll|l|l}
$h$ & $\operatorname{deg} \chi_{h}$ & $h$ & $\operatorname{deg} \chi_{h}$ & $h$ & $I_{h}$ & exceptions \\
\hline 1 & 1 & 2 & $q(q+1)$ & $1,2,3$ & - & \\
3 & $q^{3}$ & 4 & $q^{2}+q+1$ & 4,5 & $n \in \mathbb{Z}_{q-1}$ & $(q-1) \mid n$ \\
5 & $q\left(q^{2}+q+1\right)$ & 6 & $(q+1)\left(q^{2}+q+1\right)$ & 6 & $(n, m) \in \mathbb{Z}_{q-1}^{2}$ & $(q-1) \mid(n-m)$ \\
7 & $(q-1)\left(q^{2}+q+1\right)$ & 8 & $(q-1)^{2}(q+1)$ & 7 & $n \in \mathbb{Z}_{q^{2}-1}$ & $(q+1) \mid n$ \\
& & 8 & $n \in \mathbb{Z}_{q^{2}+q+1}$ & $\left(q^{2}+q+1\right) \mid n$
\end{tabular}

Table (11.5.3-B). Some $S y l_{p}$-decompositions of irreducible characters for $S L(3, q)$ with the condition that $3 \nmid(q-1)$.

\begin{tabular}{c|c||c|c||c|c||c|c||c|c}
$h$ & $\mathbf{v}$ & $h$ & $\mathbf{v}$ & $h$ & $\mathbf{v}$ & $h$ & $\mathbf{v}$ & $h$ & $\mathbf{v}$ \\
\hline 3 & $(1,7)$ & 4 & $(1,2)$ & 5 & $(2,3)$ & 5 & $(4,7)$ & 6 & $(4,5)$
\end{tabular}


Table [11.5]3-C). The $S y l_{p}$-vanishing characters of degree $S t(1)$ for $S L(3, q)$ with the condition that $3 \nmid(q-1)$.

$$
\begin{array}{c|c||c|c}
j & \mathbf{v} & j & \mathbf{v} \\
\hline 1 & (1,7) & 2 & (3)
\end{array}
$$

11.6. Groups $U(4, q)$.

Table (11.6 A). CHEviE sets $\mathbf{X}_{h}$, their degrees and parameters for $U(4, q)$.

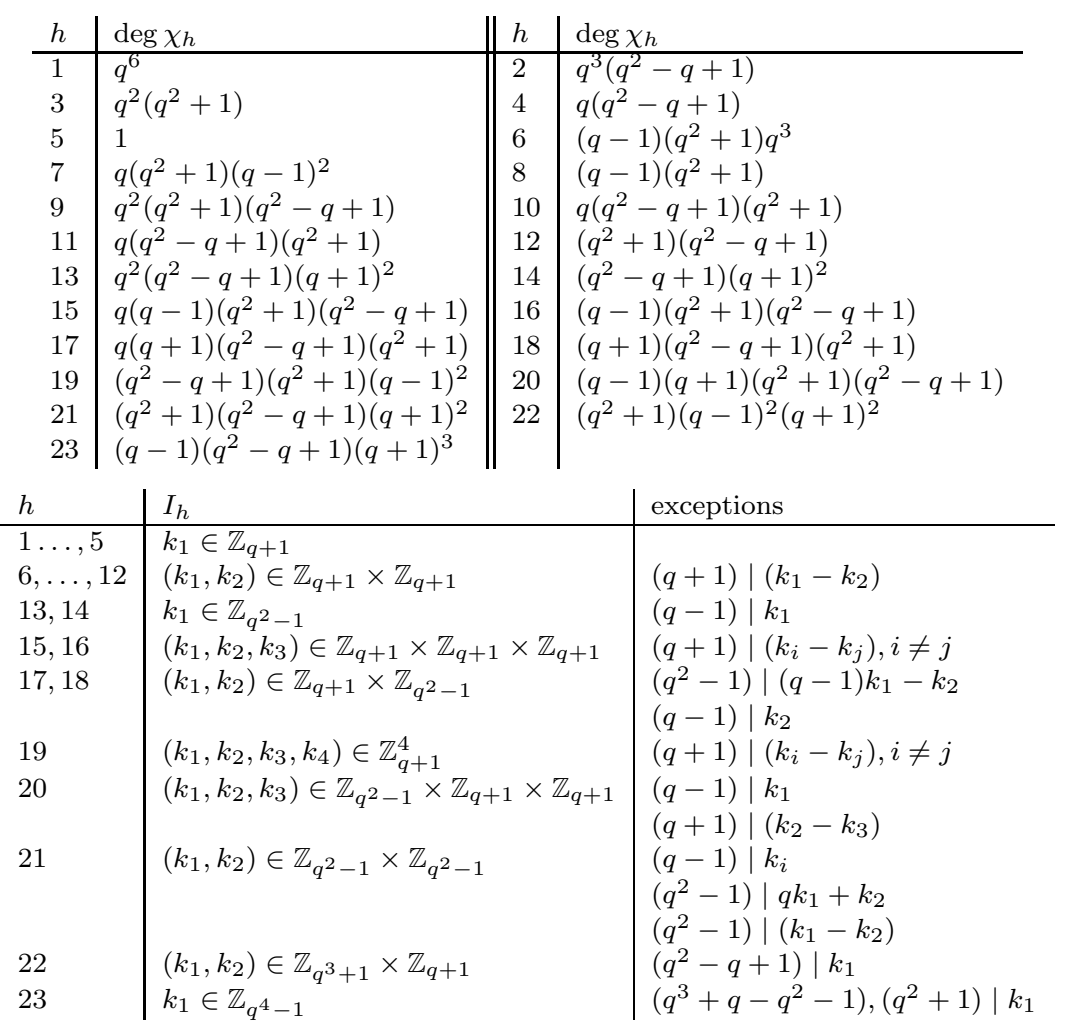

Table (11.6 B). Some $S y l_{p}$-decompositions of irreducible characters for $U(4, q)$.

\begin{tabular}{c|c||c|c||c|c||c|c||c|c||c|c}
$h$ & $\mathbf{v}$ & $h$ & $\mathbf{v}$ & $h$ & $\mathbf{v}$ & $h$ & $\mathbf{v}$ & $h$ & $\mathbf{v}$ & $h$ & $\mathbf{v}$ \\
\hline 10 & $(11)$ & 1 & $(2,6)$ & 3 & $(8,12)$ & 4 & $(5,8)$ & 6 & $(7,15)$ & 9 & $(3,6)$ \\
9 & $(11,15)$ & 9 & $(12,20)$ & 11 & $(2,4)$ & 11 & $(3,7)$ & 11 & $(12,16)$ & 13 & $(14,23)$ \\
15 & $(16,19)$ & 16 & $(7,8)$ & 17 & $(9,11)$ & 17 & $(18,20)$ & 18 & $(11,12)$ & 20 & $(6,8)$ \\
20 & $(15,16)$ & 21 & $(13,14)$ & 21 & $(17,18)$ & 22 & $(7,7,7,19)$ & 14 & $(5,5,8,8,12)$ & &
\end{tabular}

Table (11.6-C). The $S y l_{p}$-vanishing characters of degree $S t(1)$ for $U(4, q)$.

\begin{tabular}{c|c||c|c||c|c||c|c||c|c}
$j$ & $\mathbf{v}$ & $j$ & $\mathbf{v}$ & $j$ & $\mathbf{v}$ & $j$ & $\mathbf{v}$ & $j$ & $\mathbf{v}$ \\
\hline 1 & $(1)$ & 2 & $(2,6)$ & 3 & $(2,7,7,8,19)$ & 4 & $(2,7,15)$ & 5 & $(2,7,16,19)$
\end{tabular}




\subsection{Groups $U(5, q)$.}

Table (11.7 A). CHEVIE sets $\mathbf{X}_{h}$, their degrees and parameters for $U(5, q)$.

\begin{tabular}{l|l||l|l}
$h$ & $\operatorname{deg} \chi_{h}$ & $h$ & $\operatorname{deg} \chi_{h}$ \\
\hline 1 & $q^{10}$ & 2 & $q^{6}(q-1)\left(q^{2}+1\right)$ \\
5 & $q^{2}\left(q^{4}-q^{3}+q^{2}-q+1\right)$ & 8 & $q^{6}\left(q^{4}-q^{3}+q^{2}-q+1\right)$ \\
9 & $q^{3}\left(q^{2}-q+1\right)\left(q^{4}-q^{3}+q^{2}-q+1\right)$ & 15 & $q^{2}(q-1)\left(q^{2}+1\right)\left(q^{4}-q^{3}+q^{2}-q+1\right)$ \\
16 & $q(q-1)\left(q^{2}+1\right)\left(q^{4}-q^{3}+q^{2}-q+1\right)$ & 19 & $q^{3}(q-1)\left(q^{2}+1\right)\left(q^{4}-q^{3}+q^{2}-q+1\right)$ \\
20 & $q\left(q^{2}+1\right)\left(q^{4}-q^{3}+q^{2}-q+1\right)(q-1)^{2}$ & 21 & $(q-1)\left(q^{2}+1\right)\left(q^{4}-q^{3}+q^{2}-q+1\right)$ \\
31 & $q(q-1)\left(q^{2}-q+1\right)\left(q^{4}-q^{3}+q^{2}-q+1\right)\left(q^{2}+1\right)$ & 32 & $(q-1)\left(q^{2}-q+1\right)\left(q^{4}-q^{3}+q^{2}-q+1\right)\left(q^{2}+1\right)$ \\
37 & $\left(q^{2}-q+1\right)\left(q^{4}-q^{3}+q^{2}-q+1\right)\left(q^{2}+1\right)(q-1)^{2}$ &
\end{tabular}

\begin{tabular}{l|l|l}
$h$ & $I_{h}$ & exceptions \\
\hline $1,2,5$ & $k_{1} \in \mathbb{Z}_{+1}$ & \\
$8,9,15,16$ & $\left(k_{1}, k_{2}\right) \in \mathbb{Z}_{q+1}^{2}$ & $(q+1) \mid k_{1}-k_{2}$ \\
$19,20,21$ & $\left(k_{1}, k_{2}, k_{3}\right) \in \mathbb{Z}_{q+1}^{3}$ & $(q+1) \mid k_{i}-k_{j}, i \neq j$ \\
31,32 & $\left(k_{1}, k_{2}, k_{3}, k_{4}\right) \in \mathbb{Z}_{q+1}^{4}$ & $(q+1) \mid k_{i}-k_{j}, i \neq j$ \\
37 & $\left(k_{1}, k_{2}, k_{3}, k_{4}, k_{5}\right) \in \mathbb{Z}_{q+1}^{5}$ & $(q+1) \mid k_{i}-k_{j}, i \neq j$
\end{tabular}

Table (11.7 B). Some $S y l_{p}$-decompositions of irreducible characters for $U(5, q)$.

\begin{tabular}{c|c||c|c||c|c||c|c||c|c||c|c}
$h$ & $\mathbf{v}$ & $h$ & $\mathbf{v}$ & $h$ & $\mathbf{v}$ & $h$ & $\mathbf{v}$ & $h$ & $\mathbf{v}$ & $h$ & $\mathbf{v}$ \\
\hline 26 & $(27)$ & 1 & $(2,8)$ & 3 & $(11,16)$ & 4 & $(6,17)$ & 8 & $(9,19)$ & 9 & $(5,15)$ \\
10 & $(3,5)$ & 10 & $(16,17)$ & 10 & $(21,28)$ & 11 & $(12,21)$ & 12 & $(6,7)$ & 13 & $(3,8)$ \\
13 & $(14,19)$ & 13 & $(15,25)$ & 14 & $(2,4)$ & 14 & $(3,9)$ & 14 & $(10,15)$ & 14 & $(16,27)$ \\
14 & $(17,23)$ & 15 & $(16,20)$ & 17 & $(5,11)$ & 17 & $(18,21)$ & 18 & $(5,12)$ & 19 & $(20,31)$ \\
22 & $(13,14)$ & 22 & $(23,33)$ & 23 & $(2,6)$ & 23 & $(15,16)$ & 24 & $(17,18)$ & 25 & $(10,19)$ \\
25 & $(27,31)$ & 25 & $(28,38)$ & 27 & $(9,11)$ & 27 & $(10,20)$ & 27 & $(15,17)$ & 27 & $(28,32)$ \\
28 & $(16,18)$ & 29 & $(30,42)$ & 31 & $(32,37)$ & 32 & $(20,21)$ & 33 & $(13,17)$ & 33 & $(25,27)$ \\
33 & $(34,38)$ & 34 & $(14,18)$ & 34 & $(23,24)$ & 34 & $(27,28)$ & 35 & $(36,40)$ & 38 & $(19,21)$ \\
38 & $(31,32)$ & 39 & $(22,24)$ & 39 & $(29,30)$ & 39 & $(33,34)$ & 41 & $(35,36)$ & 36 & $(2,6,16,21)$ \\
40 & $(20,20,20,37)$ & & & & & & & & &
\end{tabular}

Table (11.7 $\mathrm{C})$. The $S y l_{p}$-vanishing characters of degree $S t(1)$ for $U(5, q)$.

\begin{tabular}{c|c||c|c||c|c}
$j$ & $\mathbf{v}$ & $j$ & $\mathbf{v}$ & $j$ & $\mathbf{v}$ \\
\hline 1 & $(2,5,15,19)$ & 2 & $(2,5,15,20,20,21,37)$ & 3 & $(2,5,15,20,31)$ \\
4 & $(2,5,15,20,32,37)$ & 5 & $(2,5,16,19,20)$ & 6 & $(2,5,16,20,20,20,21,37)$ \\
7 & $(2,5,16,20,20,31)$ & 8 & $(2,5,16,20,20,32,37)$ & 9 & $(2,5,16,21,40)$ \\
10 & $(2,9,19)$ & 11 & $(2,9,20,20,21,37)$ & 12 & $(2,9,20,31)$ \\
13 & $(2,9,20,32,37)$ & 14 & $(2,8)$ & 15 & $(1)$
\end{tabular}

11.8. Groups $S p(4, q)$.

Table (11.8,1-A). CHEviE sets $\mathbf{X}_{h}$, their degrees and parameters for $S p(4, q)$ with $q$ even.

\begin{tabular}{l|l||l|l||l|l}
$h$ & $\operatorname{deg} \chi_{h}$ & $h$ & $\operatorname{deg} \chi_{h}$ & $h$ & $\operatorname{deg} \chi_{h}$ \\
\hline 1 & 1 & 2 & $\frac{1}{2} q(q+1)^{2}$ & 3,4 & $\frac{1}{2} q\left(q^{2}+1\right)$ \\
5 & $\frac{1}{2} q(q-1)^{2}$ & 6 & $q^{4}$ & 7 & $(q+1)\left(q^{2}+1\right)$ \\
8 & $q(q+1)\left(q^{2}+1\right)$ & 9 & $(q-1)\left(q^{2}+1\right)$ & 10 & $q(q-1)\left(q^{2}+1\right)$ \\
11 & $(q+1)\left(q^{2}+1\right)$ & 12 & $q(q+1)\left(q^{2}+1\right)$ & 13 & $(q-1)\left(q^{2}+1\right)$ \\
14 & $q(q-1)\left(q^{2}+1\right)$ & 15 & $(q+1)^{2}\left(q^{2}+1\right)$ & 16,17 & $(q-1)(q+1)\left(q^{2}+1\right)$ \\
18 & $(q-1)^{2}(q+1)^{2}$ & 19 & $(q-1)^{2}\left(q^{2}+1\right)$ & &
\end{tabular}




\begin{tabular}{l|l|l}
$h$ & $I_{h}$ & exceptions \\
\hline $1, \ldots, 6$ & - & \\
$7,8,11,12$ & $k \in \mathbb{Z}_{q-1}$ & $(q-1) \mid k$ \\
$9,10,13,14$ & $k \in \mathbb{Z}_{q+1}$ & $(q+1) \mid k$ \\
15 & $(k, l) \in \mathbb{Z}_{q-1}^{2}$ & $(q-1) \mid k \pm l$ \\
16 & $k \in \mathbb{Z}_{q^{2}-1}$ & $(q \pm 1) \mid k$ \\
17 & $(k, l) \in \mathbb{Z}_{q-1} \times \mathbb{Z}_{q+1}$ & $(q-1)|k,(q+1)| l$ \\
18 & $k \in \mathbb{Z}_{q^{2}+1}$ & $\left(q^{2}+1\right) \mid k$ \\
19 & $(k, l) \in \mathbb{Z}_{q+1}^{2}$ & $(q+1) \mid k \pm l$
\end{tabular}

Table (11.8,1-B). Some $S y l_{p}$-decompositions of irreducible characters for $S p(4, q)$ with $q$ even.

\begin{tabular}{c|c||c|c||c|c||c|c||c|c}
$h$ & $\mathbf{v}$ & $h$ & $\mathbf{v}$ & $h$ & $\mathbf{v}$ & $h$ & $\mathbf{v}$ & $h$ & $\mathbf{v}$ \\
\hline 8 & $(7,17)$ & 10 & $(9,19)$ & 12 & $(11,16)$ & 14 & $(13,19)$ & 15 & $(7,8)$ \\
15 & $(11,12)$ & 16 & $(13,14)$ & 17 & $(9,10)$ & 7 & $(1,2,3)$ & 11 & $(1,2,4)$ \\
6 & $(1,9,13,19)$ & 6 & $(3,5,9,19)$ & 6 & $(4,5,13,19)$ & & & &
\end{tabular}

Table (11.81-C). The $S y l_{p}$-vanishing characters of degree $S t(1)$ for $S p(4, q)$ with $q$ even.

\begin{tabular}{c|c||c|c||c|c||c|c}
$j$ & $\mathbf{v}$ & $j$ & $\mathbf{v}$ & $j$ & $\mathbf{v}$ & $j$ & $\mathbf{v}$ \\
\hline 1 & $(1,9,13,19)$ & 2 & $(1,9,14)$ & 3 & $(1,10,13)$ & 4 & $(3,5,9,19)$ \\
5 & $(3,5,10)$ & 6 & $(4,5,13,19)$ & 7 & $(4,5,14)$ & 8 & $(6)$
\end{tabular}

Table (11.82-A). CHEvie sets $\mathbf{X}_{h}$ and their degrees for $S p(4, q)$ with $q$ odd.

In this table, for reader's sake, we additionally provide the notation of [21] for the irreducible characters of $G$.

\begin{tabular}{l|l|l||l|l|l}
$h$ & {$[21$} & $\operatorname{deg} \chi_{h}$ & $h$ & $\underline{21}$ & $\operatorname{deg} \chi_{h}$ \\
\hline 1 & $\chi_{1}$ & $q^{4}-2 q^{2}+1$ & 2 & $-\chi_{2}$ & $q^{4}-1$ \\
3 & $\chi_{3}$ & $q^{4}+2 q^{3}+2 q^{2}+2 q+1$ & 4 & $\chi_{4}$ & $q^{4}-2 q^{3}+2 q^{2}-2 q+1$ \\
5 & $-\chi_{5}$ & $q^{4}-1$ & 6 & $-\chi_{6}$ & $q^{3}-q^{2}+q-1$ \\
7 & $\chi_{7}$ & $q^{4}-q^{3}+q^{2}-q$ & 8 & $\chi_{8}$ & $q^{3}+q^{2}+q+1$ \\
9 & $\chi_{9}$ & $q^{4}+q^{3}+q^{2}+q$ & 10 & $-\xi_{1}$ & $q^{3}-q^{2}+q-1$ \\
11 & $-\xi_{1}^{\prime}$ & $q^{4}-q^{3}+q^{2}-q$ & 12 & $\xi_{3}$ & $q^{3}+q^{2}+q+1$ \\
13 & $\xi_{3}^{\prime}$ & $q^{4}+q^{3}+q^{2}+q$ & 14 & $-\xi_{21}$ & $\left(q^{4}-1\right) / 2$ \\
15 & $-\xi_{22}$ & $\left(q^{4}-1\right) / 2$ & 16 & $\xi_{21}^{\prime}$ & $\left(q^{4}-2 q^{3}+2 q^{2}-2 q+1\right) / 2$ \\
17 & $\xi_{22}^{\prime}$ & $\left(q^{4}-2 q^{3}+2 q^{2}-2 q+1\right) / 2$ & 18 & $\xi_{41}$ & $\left(q^{4}+2 q^{3}+2 q^{2}+2 q+1\right) / 2$ \\
19 & $\xi_{42}$ & $\left(q^{4}+2 q^{3}+2 q^{2}+2 q+1 / 2\right.$ & 20 & $-\xi_{41}^{\prime}$ & $\left(q^{4}-1\right) / 2$ \\
21 & $-\xi_{42}^{\prime}$ & $\left(q^{4}-1\right) / 2$ & 22 & $-\Phi_{1}$ & $\left(q^{3}-q^{2}+q-1\right) / 2$ \\
23 & $-\Phi_{2}$ & $\left(q^{3}-q^{2}+q-1\right) / 2$ & 24 & $-\Phi_{3}$ & $\left(q^{4}-q^{3}+q^{2}-q\right) / 2$ \\
25 & $-\Phi_{4}$ & $\left(q^{4}-q^{3}+q^{2}-q\right) / 2$ & 26 & $\Phi_{5}$ & $\left(q^{3}+q^{2}+q+1\right) / 2$ \\
27 & $\Phi_{6}$ & $\left(q^{3}+q^{2}+q+1\right) / 2$ & 28 & $\Phi_{7}$ & $\left(q^{4}+q^{3}+q^{2}+q\right) / 2$ \\
29 & $\Phi_{8}$ & $\left(q^{4}+q^{3}+q^{2}+q\right) / 2$ & 30 & $\Phi_{9}$ & $q^{3}+q$ \\
31 & $\theta_{1}$ & $\left(q^{4}+q^{2}\right) / 2$ & 32 & $\theta_{2}$ & $\left(q^{4}+q^{2}\right) / 2$ \\
33 & $\theta_{3}$ & $\left(q^{2}+1\right) / 2$ & 34 & $\theta_{4}$ & $\left(q^{2}+1\right) / 2$ \\
35 & $-\theta_{5}$ & $\left(q^{4}-q^{2}\right) / 2$ & 36 & $-\theta_{6}$ & $\left(q^{4}-q^{2}\right) / 2$ \\
37 & $-\theta_{7}$ & $\left(q^{2}-1\right) / 2$ & 38 & $-\theta_{8}$ & $\left(q^{2}-1\right) / 2$ \\
39 & $\theta_{9}$ & $\left(q^{3}+2 q^{2}+q\right) / 2$ & 40 & $\theta_{10}$ & $\left(q^{3}-2 q^{2}+q\right) / 2$ \\
41 & $\theta_{11}$ & $\left(q^{3}+q\right) / 2$ & 42 & $\theta_{12}$ & $\left(q^{3}+q\right) / 2$ \\
43 & $\theta_{13}$ & $q^{4}$ & 44 & $\theta_{0}$ & 1
\end{tabular}


Table (11.8,2-B). Some $S y l_{p}$-decompositions of irreducible characters for $S p(4, q)$ with $q$ odd.

\begin{tabular}{c|c||c|c||c|c||c|c||c|c||c|c}
$h$ & $\mathbf{v}$ & $h$ & $\mathbf{v}$ & $h$ & $\mathbf{v}$ & $h$ & $\mathbf{v}$ & $h$ & $\mathbf{v}$ & $h$ & $\mathbf{v}$ \\
\hline 2 & $(6,7)$ & 3 & $(8,9)$ & 3 & $(12,13)$ & 3 & $(18,19)$ & 4 & $(16,17)$ & 5 & $(10,11)$ \\
5 & $(14,15)$ & 5 & $(20,21)$ & 7 & $(4,6)$ & 9 & $(2,8)$ & 10 & $(22,23)$ & 11 & $(4,10)$ \\
11 & $(14,16)$ & 11 & $(15,17)$ & 11 & $(24,25)$ & 12 & $(26,27)$ & 13 & $(5,12)$ & 13 & $(18,20)$ \\
13 & $(19,21)$ & 13 & $(28,29)$ & 14 & $(10,17)$ & 14 & $(23,24)$ & 14 & $(35,38)$ & 15 & $(10,16)$ \\
15 & $(22,25)$ & 15 & $(36,37)$ & 18 & $(12,21)$ & 18 & $(26,28)$ & 19 & $(12,20)$ & 19 & $(27,29)$ \\
20 & $(22,24)$ & 20 & $(35,37)$ & 21 & $(23,25)$ & 21 & $(36,38)$ & 22 & $(37,40)$ & 23 & $(38,40)$ \\
24 & $(17,22)$ & 25 & $(16,23)$ & 26 & $(34,42)$ & 27 & $(33,42)$ & 28 & $(15,26)$ & 28 & $(21,27)$ \\
28 & $(25,30)$ & 28 & $(31,41)$ & 28 & $(36,39)$ & 29 & $(14,27)$ & 29 & $(20,26)$ & 29 & $(24,30)$ \\
29 & $(32,41)$ & 29 & $(35,39)$ & 30 & $(22,26)$ & 30 & $(23,27)$ & 30 & $(39,40)$ & 30 & $(41,42)$ \\
31 & $(25,42)$ & 32 & $(24,42)$ & 33 & $(37,44)$ & 34 & $(38,44)$ & 35 & $(24,40)$ & 36 & $(25,40)$ \\
39 & $(26,37)$ & 39 & $(27,38)$ & 41 & $(22,34)$ & 41 & $(23,33)$ & 43 & $(31,35)$ & 43 & $(32,36)$
\end{tabular}

$$
\begin{array}{c|c}
h & \mathbf{v} \\
\hline 1 & (16,17,40,40,40,40)
\end{array}
$$

Table (11.82-C). The $S y l_{p}$-vanishing characters of degree $S t(1)$ for $S p(4, q)$ with $q$ odd.

\begin{tabular}{|c|c|c|c|c|c|}
\hline$j$ & $\mathbf{v}$ & $j$ & $\mathbf{V}$ & $j$ & $\mathbf{V}$ \\
\hline 1 & $(4,6,10,44)$ & 2 & $(4,6,22,23,44)$ & 3 & $(4,6,22,34,40)$ \\
\hline 4 & $(4,6,22,38,40,44)$ & 5 & $(4,6,23,33,40)$ & 6 & $(4,6,23,37,40,44)$ \\
\hline 7 & $(4,6,33,38,40,40)$ & 8 & $(4,6,34,37,40,40)$ & 9 & $(4,6,37,38,40,40,44)$ \\
\hline 10 & $(4,6,40,41)$ & 11 & $(4,10,40,42)$ & 12 & $(4,22,23,40,42)$ \\
\hline 13 & $(4,22,38,40,40,42)$ & 14 & $(4,23,37,40,40,42)$ & 15 & $(4,37,38,40,40,40,42)$ \\
\hline 16 & $(6,10,16,17,44)$ & 17 & $(6,11,44)$ & 18 & $(6,14,16,44)$ \\
\hline 19 & $(6,15,17,44)$ & 20 & $(6,16,17,22,23,44)$ & 21 & $(6,16,17,22,34,40)$ \\
\hline 22 & $(6,16,17,22,38,40,44)$ & 23 & $(6,16,17,23,33,40)$ & 24 & $(6,16,17,23,37,40,44)$ \\
\hline 25 & $(6,16,17,33,38,40,40)$ & 26 & $(6,16,17,34,37,40,40)$ & 27 & $(6,16,17,37,38,40,40,44)$ \\
\hline 28 & $(6,16,17,40,41)$ & 29 & $(6,16,23,24,44)$ & 30 & $(6,16,24,34,40)$ \\
\hline 31 & $(6,16,24,38,40,44)$ & 32 & $(6,16,34,35)$ & 33 & $(6,16,35,38,44)$ \\
\hline 34 & $(6,17,22,25,44)$ & 35 & $(6,17,25,33,40)$ & 36 & $(6,17,25,37,40,44)$ \\
\hline 37 & $(6,17,33,36)$ & 38 & $(6,17,36,37,44)$ & 39 & $(6,24,25,44)$ \\
\hline 40 & $(7,10,44)$ & 41 & $(7,22,23,44)$ & 42 & $(7,22,34,40)$ \\
\hline 43 & $(7,22,38,40,44)$ & 44 & $(7,23,33,40)$ & 45 & $(7,23,37,40,44)$ \\
\hline 46 & $(7,33,38,40,40)$ & 47 & $(7,34,37,40,40)$ & 48 & $(7,37,38,40,40,44)$ \\
\hline 49 & $(7,40,41)$ & 50 & $(10,16,17,40,42)$ & 51 & $(11,40,42)$ \\
\hline 52 & $(14,16,40,42)$ & 53 & $(15,17,40,42)$ & 54 & $(16,17,22,23,40,42)$ \\
\hline 55 & $(16,17,22,38,40,40,42)$ & 56 & $(16,17,23,37,40,40,42)$ & 57 & $(16,17,37,38,40,40,40,42)$ \\
\hline 58 & $(16,23,24,40,42)$ & 59 & $(16,23,32,40)$ & 60 & $(16,23,35,42)$ \\
\hline 61 & $(16,24,38,40,40,42)$ & 62 & $(16,32,38,40,40)$ & 63 & $(16,35,38,40,42)$ \\
\hline 64 & $(17,22,25,40,42)$ & 65 & $(17,22,31,40)$ & 66 & $(17,22,36,42)$ \\
\hline 67 & $(17,25,37,40,40,42)$ & 68 & $(17,31,37,40,40)$ & 69 & $(17,36,37,40,42)$ \\
\hline 70 & $(24,25,40,42)$ & 71 & $(24,31,40)$ & 72 & $(24,36,42)$ \\
\hline 73 & $(25,32,40)$ & 74 & $(25,35,42)$ & 75 & $(31,35)$ \\
\hline 76 & $(32,36)$ & 77 & $(43)$ & & \\
\hline
\end{tabular}


Table (11.8,2-D). The $p$-vanishing characters of degree $S t(1)$ and their values for $S p(4, q)$ with $q$ odd and $7 \mid(q+1)$.

In view of Lemma 7.9 we have the character $\psi=1_{G}+\chi_{4}(a, b)+\chi_{6}(c)+\chi_{10}(d)$. This character differs from $S t$ only on the following classes:

\begin{tabular}{l||l|l}
$C_{i}$ & $S t$ & $\psi$ \\
\hline$B_{2}(i)$ & -1 & $1-\beta_{i c}$ \\
$B_{4}(i, j)$ & 1 & $1+\beta_{i a} \beta_{j b}+\beta_{i b} \beta_{j a}-\beta_{i c} \beta_{j c}-\beta_{i d}-\beta_{j d}$ \\
$B_{5}(i)$ & -1 & $1-\beta_{i d}$ \\
$B_{6}(i)$ & $-q$ & $q\left(1-\beta_{2 i c}\right)$ \\
$C_{1}(i)$ & $-q$ & $q\left(1-\beta_{i d}\right)$
\end{tabular}

Here, $\beta_{t}=2 \operatorname{Re}\left(\xi_{1}^{t}\right)$.

Table (11.8 3-A). CHEvIE sets $\mathbf{X}_{h}$ and their degrees for $C S p(4, q)$ with $q$ odd.

Here, for the reader's sake, we also recall the notation of [20] for irreducible characters of $H=C S p(4, q)$.

\begin{tabular}{l|l|l||l|l|l}
$h$ & 20 & $\operatorname{deg} \chi_{h}$ & $h$ & 20 & $\operatorname{deg} \chi_{h}$ \\
\hline 1 & $X_{1}(\lambda, \mu)$ & $(q+1)^{2}\left(q^{2}+1\right)$ & 2 & $X_{2}(\Lambda)$ & $q^{4}-1$ \\
3 & $X_{3}(\Lambda, \nu)$ & $q^{4}-1$ & 4 & $X_{4}(\theta)$ & $\left(q^{2}-1\right)^{2}$ \\
5 & $X_{5}(\Lambda, \omega)$ & $\left(q^{2}+1\right)(q-1)^{2}$ & 6 & $X_{1}(\lambda)$ & $(q+1)\left(q^{2}+1\right)$ \\
7 & $X_{2}(\lambda)$ & $q(q+1)\left(q^{2}+1\right)$ & 8 & $X_{3}(\lambda)$ & $(q+1)\left(q^{2}+1\right)$ \\
9 & $X_{4}(\lambda)$ & $q(q+1)\left(q^{2}+1\right)$ & 10 & $X_{5}(\omega)$ & $(q-1)\left(q^{2}+1\right)$ \\
11 & $X_{6}(\omega)$ & $q(q-1)\left(q^{2}+1\right)$ & 12 & $X_{7}(\Lambda)$ & $(q-1)\left(q^{2}+1\right)$ \\
13 & $X_{8}(\Lambda)$ & $q(q-1)\left(q^{2}+1\right)$ & 14 & $\tau_{1}$ & $q^{2}+1$ \\
15 & $\tau_{2}$ & $q\left(q^{2}+1\right)$ & 16 & $\tau_{3}$ & $q^{2}\left(q^{2}+1\right)$ \\
17 & $\tau_{4}\left(\lambda^{\prime}\right)$ & $q^{2}-1$ & 18 & $\tau_{5}\left(\lambda^{\prime}\right)$ & $q^{2}\left(q^{2}-1\right)$ \\
19 & $\theta_{1}$ & $q(q+1)^{2} / 2$ & 20 & $\theta_{2}$ & $q(q-1)^{2} / 2$ \\
21 & $\theta_{3}$ & $q\left(q^{2}+1\right) / 2$ & 22 & $\theta_{4}$ & $q\left(q^{2}+1\right) / 2$ \\
23 & $\theta_{5}$ & $q^{4}$ & 24 & $\theta_{0}(\lambda)$ & 1
\end{tabular}

Table (11.8,3-B). Some $S y l_{p}$-decompositions of irreducible characters for $C \operatorname{Sp}(4, q)$ with $q$ odd.

\begin{tabular}{c|c||c|c||c|c||c|c||c|c}
$h$ & $\mathbf{v}$ & $h$ & $\mathbf{v}$ & $h$ & $\mathbf{v}$ & $h$ & $\mathbf{v}$ & $h$ & $\mathbf{v}$ \\
\hline 1 & $(6,7)$ & 1 & $(8,9)$ & 2 & $(10,11)$ & 3 & $(12,13)$ & 3 & $(17,18)$ \\
7 & $(3,6)$ & 8 & $(14,15)$ & 9 & $(2,8)$ & 9 & $(15,16)$ & 11 & $(5,10)$ \\
13 & $(5,12)$ & 15 & $(10,14)$ & 15 & $(19,20)$ & 15 & $(21,22)$ & 16 & $(2,14)$ \\
16 & $(11,15)$ & 18 & $(4,17)$ & 6 & $(14,22,22)$ & 6 & $(19,22,24)$ & 12 & $(17,20,20)$ \\
14 & $(17,24,24)$ & 19 & $(17,22,24)$ & 21 & $(17,20,24)$ & & &
\end{tabular}

\begin{tabular}{c|c||c|c||c|c}
$h$ & $\mathbf{v}$ & $h$ & $\mathbf{v}$ & $h$ & $\mathbf{v}$ \\
\hline 4 & $(5,20,20,20,20)$ & 23 & $(5,10,17,20,20,24)$ & 23 & $(5,17,20,20,20,22)$
\end{tabular}

Table (11.8,3-C). The $S y l_{p}$-vanishing characters of degree $\operatorname{St}(1)$ for $C S p(4, q)$ with $q$ odd.

\begin{tabular}{c|c||c|c||c|c||c|c}
$j$ & $\mathbf{v}$ & $j$ & $\mathbf{v}$ & $j$ & $\mathbf{v}$ & $j$ & $\mathbf{v}$ \\
\hline 1 & $(5,10,12,24)$ & 2 & $(5,10,17,20,20,24)$ & 3 & $(5,10,20,21)$ & 4 & $(5,12,20,22)$ \\
5 & $(5,17,20,20,20,22)$ & 6 & $(10,13,24)$ & 7 & $(11,12,24)$ & 8 & $(11,17,20,20,24)$ \\
9 & $(11,20,21)$ & 10 & $(13,20,22)$ & 11 & $(23)$ &
\end{tabular}




\subsection{Groups ${ }^{3} D_{4}(q)$.}

Table (11.9, 1-A). CHEVIE sets $\mathbf{X}_{h}$, their degrees and parameters for ${ }^{3} D_{4}(q)$ with $q$ even.

\begin{tabular}{|c|c|c|c|c|}
\hline$h$ & \multicolumn{2}{|l|}{$\operatorname{deg} \chi_{h}$} & \begin{tabular}{|l|l}
$h$ \\
\end{tabular} & $\operatorname{deg} \chi_{h}$ \\
\hline 1 & \multirow{2}{*}{\multicolumn{2}{|c|}{$\begin{array}{l}1 \\
q^{3}(q+1)^{2}\left(q^{4}-q^{2}+1\right) / 2\end{array}$}} & 2 & $q\left(q^{4}-q^{2}+1\right)$ \\
\hline 3 & & & 4 & $q^{3}(q+1)^{2}\left(q^{2}-q+1\right)^{2} / 2$ \\
\hline 5 & \multicolumn{2}{|l|}{$q^{3}(q-1)^{2}\left(q^{2}+q+1\right)^{2} / 2$} & 6 & $q^{3}(q-1)^{2}\left(q^{4}-q^{2}+1\right) / 2$ \\
\hline 7 & \multicolumn{2}{|c|}{$q^{7}\left(q^{4}-q^{2}+1\right)$} & 8 & \\
\hline 9 & \multicolumn{2}{|c|}{$(q+1)\left(q^{2}+q+1\right)\left(q^{2}-q+1\right)\left(q^{4}-q^{2}+1\right)$} & 10 & $q^{3}(q+1)\left(q^{2}-q+1\right)\left(q^{2}+q+1\right)\left(q^{4}-q^{2}+1\right)$ \\
\hline 11 & \multirow{2}{*}{\multicolumn{2}{|c|}{$\begin{array}{l}(q+1)\left(q^{4}-q^{2}+1\right)\left(q^{2}-q+1\right)^{2} \\
q^{3}(q+1)\left(q^{4}-q^{2}+1\right)\left(q^{2}-q+1\right)^{2}\end{array}$}} & 12 & $q\left(q^{4}-q^{2}+1\right)(q+1)^{2}\left(q^{2}-q+1\right)^{2}$ \\
\hline 13 & & & 14 & $(q+1)\left(q^{2}+q+1\right)\left(q^{4}-q^{2}+1\right)\left(q^{2}-q+1\right)^{2}$ \\
\hline 15 & \multicolumn{2}{|c|}{$q(q+1)\left(q^{2}+q+1\right)\left(q^{4}-q^{2}+1\right)\left(q^{2}-q+1\right)^{2}$} & 16 & $\left(q^{2}+q+1\right)\left(q^{4}-q^{2}+1\right)(q+1)^{2}\left(q^{2}-q+1\right)^{2}$ \\
\hline 17 & \multirow{2}{*}{\multicolumn{2}{|c|}{$\begin{array}{l}(q-1)\left(q^{2}+q+1\right)\left(q^{2}-q+1\right)\left(q^{4}-q^{2}+1\right) \\
(q-1)(q+1)\left(q^{2}+q+1\right)\left(q^{2}-q+1\right)^{2}\left(q^{4}-q^{2}+1\right)\end{array}$}} & 18 & $q^{3}(q-1)\left(q^{2}-q+1\right)\left(q^{2}+q+1\right)\left(q^{4}-q^{2}+1\right)$ \\
\hline 19 & & & 20 & $(q-1)\left(q^{4}-q^{2}+1\right)\left(q^{2}+q+1\right)^{2}$ \\
\hline 21 & \multirow{2}{*}{\multicolumn{2}{|c|}{$\begin{array}{l}q\left(q^{4}-q^{2}+1\right)(q-1)^{2}\left(q^{2}+q+1\right)^{2} \\
(q-1)\left(q^{2}-q+1\right)\left(q^{4}-q^{2}+1\right)\left(q^{2}+q+1\right)^{2}\end{array}$}} & 22 & $q^{3}(q-1)\left(q^{4}-q^{2}+1\right)\left(q^{2}+q+1\right)^{2}$ \\
\hline 23 & & & 24 & $q(q-1)\left(q^{2}-q+1\right)\left(q^{4}-q^{2}+1\right)\left(q^{2}+q+1\right)^{2}$ \\
\hline 25 & \multicolumn{2}{|c|}{$(q-1)(q+1)\left(q^{2}+q+1\right)^{2}\left(q^{2}-q+1\right)\left(q^{4}-q^{2}+1\right)$} & 26 & $(q-1)^{2}(q+1)^{2}\left(q^{2}-q+1\right)^{2}\left(q^{4}-q^{2}+1\right)$ \\
\hline 27 & \multirow{2}{*}{\multicolumn{2}{|c|}{$\begin{array}{l}(q-1)^{2}(q+1)^{2}\left(q^{2}+q+1\right)^{2}\left(q^{4}-q^{2}+1\right) \\
\left(q^{2}-q+1\right)\left(q^{4}-q^{2}+1\right)(q-1)^{2}\left(q^{2}+q+1\right)^{2}\end{array}$}} & 28 & $(q-1)^{2}(q+1)^{2}\left(q^{2}+q+1\right)^{2}\left(q^{2}-q+1\right)^{2}$ \\
\hline 29 & & & & \\
\hline & $h$ & $I_{h}$ & \multicolumn{2}{|c|}{ exceptions } \\
\hline & $1, \ldots, 8$ & - & \multirow{2}{*}{\multicolumn{2}{|c|}{$(q-1) \mid k$}} \\
\hline & 9,10 & $k \in \mathbb{Z}_{q-1}$ & & \\
\hline & $11,12,13$ & $k \in\left(q^{2}+q+1\right)$ & \multicolumn{2}{|c|}{$\left(q^{2}+q+1\right) \mid k$} \\
\hline & 14,15 & $k \in \mathbb{Z}_{q^{3}-1}$ & \multirow{2}{*}{\multicolumn{2}{|c|}{$\begin{array}{l}(q-1) \mid k \\
\text { many }\end{array}$}} \\
\hline & & $(k, l) \in \mathbb{Z}_{q^{3}-1} \times \mathbb{Z}_{q-1}$ & & \\
\hline & 17,18 & $k \in \mathbb{Z}_{q+1}$ & \\
\hline & & $k \in \mathbb{Z}_{(q+1)\left(q^{3}-1\right)}$ & \multirow{2}{*}{\multicolumn{2}{|c|}{$\begin{array}{l}(q+1),\left(q^{3}-1\right) \mid k \\
\left(q^{2}-q+1\right) \mid k\end{array}$}} \\
\hline & $20,21,22$ & $k \in \mathbb{Z}_{q^{2}-q+1}$ & & \\
\hline & 23,24 & $k \in \mathbb{Z}_{q^{3}+1}^{1}$ & \multicolumn{2}{|c|}{$(q+1) \mid k$} \\
\hline & 25 & $k \in \mathbb{Z}_{(q-1)\left(q^{3}+1\right)}$ & \multirow{2}{*}{\multicolumn{2}{|c|}{$\begin{array}{l}(q-1)\left(q^{3}+1\right) \mid k \\
\text { many }\end{array}$}} \\
\hline & 26 & $(k, l) \in \mathbb{Z}_{q^{2}+q+1}^{2}$ & & \\
\hline & 27 & $(k, l) \in \mathbb{Z}_{q^{2}-q+1}^{2}$ & \\
\hline & 28 & $k \in \mathbb{Z}_{q^{4}-q^{2}+1}$ & \multirow{2}{*}{\multicolumn{2}{|c|}{$\begin{array}{l}\left(q^{4}-q^{2}+1\right) \mid k \\
\text { many }\end{array}$}} \\
\hline & 29 & $(k, l) \in \mathbb{Z}_{q^{3}+1} \times \mathbb{Z}_{q+1}$ & & \\
\hline
\end{tabular}

Table (11.9,1-B). Some $S y l_{p}$-decompositions of irreducible characters for ${ }^{3} D_{4}(q)$ with $q$ even.

\begin{tabular}{c|c||c|c||c|c||c|c||c|c}
$h$ & $\mathbf{v}$ & $h$ & $\mathbf{v}$ & $h$ & $\mathbf{v}$ & $h$ & $\mathbf{v}$ & $h$ & $\mathbf{v}$ \\
\hline 10 & $(9,25)$ & 13 & $(11,19)$ & 14 & $(11,12)$ & 15 & $(12,13)$ & 15 & $(14,19)$ \\
16 & $(9,10)$ & 16 & $(14,15)$ & 18 & $(17,29)$ & 19 & $(17,18)$ & 22 & $(21,24)$ \\
23 & $(20,21)$ & 24 & $(23,29)$ & 25 & $(20,22)$ & 25 & $(23,24)$ & 9 & $(1,2,3,4)$ \\
27 & $(21,21,21,29)$ & 8 & $(1,17,20,21,29)$ & 8 & $(5,6,7,17,29)$ & & & &
\end{tabular}

Table (11.9) 1-C). The $S y l_{p}$-vanishing characters of degree $S t(1)$ for ${ }^{3} D_{4}(q)$ with $q$ even.

\begin{tabular}{c|c||c|c||c|c||c|c}
$j$ & $\mathbf{v}$ & $j$ & $\mathbf{v}$ & $j$ & $\mathbf{v}$ & $j$ & $\mathbf{v}$ \\
\hline 1 & $(1,17,20,21,29)$ & 2 & $(1,17,23,29)$ & 3 & $(1,17,24)$ & 4 & $(1,18,20,21)$ \\
5 & $(1,18,23)$ & 6 & $(2,5,6,20,21,29)$ & 7 & $(2,5,6,23,29)$ & 8 & $(2,5,6,24)$ \\
9 & $(5,6,7,17,29)$ & 10 & $(5,6,7,18)$ & 11 & $(8)$ & &
\end{tabular}


Table (11.9) 2-A). CHEviE sets $\mathbf{X}_{h}$, their degrees and parameters for ${ }^{3} D_{4}(q)$ with $q$ odd.

\begin{tabular}{l|l||l|l}
$h$ & $\operatorname{deg} \chi_{h}$ & $h$ & $\operatorname{deg} \chi_{h}$ \\
\hline 1 & 1 & 2 & $q\left(q^{4}-q^{2}+1\right)$ \\
3 & $q^{3}(q+1)^{2}\left(q^{4}-q^{2}+1\right) / 2$ & 4 & $q^{3}(q+1)^{2}\left(q^{2}-q+1\right)^{2} / 2$ \\
5 & $q^{3}(q-1)^{2}\left(q^{2}+q+1\right)^{2} / 2$ & 6 & $q^{3}(q-1)^{2}\left(q^{4}-q^{2}+1\right) / 2$ \\
7 & $q^{7}\left(q^{4}-q^{2}+1\right)$ & 8 & $q^{12}$ \\
9 & $\left(q^{2}+q+1\right)\left(q^{2}-q+1\right)\left(q^{4}-q^{2}+1\right)$ & 10 & $q\left(q^{2}+q+1\right)\left(q^{2}-q+1\right)\left(q^{4}-q^{2}+1\right)$ \\
11 & $q^{3}\left(q^{2}+q+1\right)\left(q^{2}-q+1\right)\left(q^{4}-q^{2}+1\right)$ & 12 & $q^{4}\left(q^{2}+q+1\right)\left(q^{2}-q+1\right)\left(q^{4}-q^{2}+1\right)$ \\
13 & $(q+1)\left(q^{2}+q+1\right)\left(q^{2}-q+1\right)\left(q^{4}-q^{2}+1\right)$ & 14 & $q^{3}(q+1)\left(q^{2}-q+1\right)\left(q^{2}+q+1\right)\left(q^{4}-q^{2}+1\right)$ \\
15 & $(q+1)\left(q^{4}-q^{2}+1\right)\left(q^{2}-q+1\right)^{2}$ & 16 & $q\left(q^{4}-q^{2}+1\right)(q+1)^{2}\left(q^{2}-q+1\right)^{2}$ \\
17 & $q^{3}(q+1)\left(q^{4}-q^{2}+1\right)\left(q^{2}-q+1\right)^{2}$ & 18 & $(q+1)\left(q^{2}+q+1\right)\left(q^{4}-q^{2}+1\right)\left(q^{2}-q+1\right)^{2}$ \\
19 & $q(q+1)\left(q^{2}+q+1\right)\left(q^{4}-q^{2}+1\right)\left(q^{2}-q+1\right)^{2}$ & 20 & $\left(q^{2}+q+1\right)\left(q^{4}-q^{2}+1\right)(q+1)^{2}\left(q^{2}-q+1\right)^{2}$ \\
21 & $(q-1)\left(q^{2}+q+1\right)\left(q^{2}-q+1\right)\left(q^{4}-q^{2}+1\right)$ & 22 & $q^{3}(q-1)\left(q^{2}-q+1\right)\left(q^{2}+q+1\right)\left(q^{4}-q^{2}+1\right)$ \\
23 & $(q-1)(q+1)\left(q^{2}+q+1\right)\left(q^{2}-q+1\right)^{2}\left(q^{4}-q^{2}+1\right)$ & 24 & $(q-1)\left(q^{4}-q^{2}+1\right)\left(q^{2}+q+1\right)^{2}$ \\
25 & $q\left(q^{4}-q^{2}+1\right)(q-1)^{2}\left(q^{2}+q+1\right)^{2}$ & 26 & $q^{3}(q-1)\left(q^{4}-q^{2}+1\right)\left(q^{2}+q+1\right)^{2}$ \\
27 & $(q-1)\left(q^{2}-q+1\right)\left(q^{4}-q^{2}+1\right)\left(q^{2}+q+1\right)^{2}$ & 28 & $q(q-1)\left(q^{2}-q+1\right)\left(q^{4}-q^{2}+1\right)\left(q^{2}+q+1\right)^{2}$ \\
29 & $(q-1)(q+1)\left(q^{2}+q+1\right)^{2}\left(q^{2}-q+1\right)\left(q^{4}-q^{2}+1\right)$ & 30 & $(q-1)^{2}(q+1)^{2}\left(q^{2}-q+1\right)^{2}\left(q^{4}-q^{2}+1\right)$ \\
31 & $(q-1)^{2}(q+1)^{2}\left(q^{2}+q+1\right)^{2}\left(q^{4}-q^{2}+1\right)$ & 32 & $(q-1)^{2}(q+1)^{2}\left(q^{2}+q+1\right)^{2}\left(q^{2}-q+1\right)^{2}$ \\
33 & $\left(q^{2}-q+1\right)\left(q^{4}-q^{2}+1\right)(q-1)^{2}\left(q^{2}+q+1\right)^{2}$ & &
\end{tabular}

\begin{tabular}{l|l|l}
$h$ & $I_{h}$ & exceptions \\
\hline $1, \ldots, 12$ & - & \\
13,14 & $k \in \mathbb{Z}_{q-1}$ & $(q-1) / 2 \mid k$ \\
$15,16,17$ & $k \in \mathbb{Z}_{q^{2}+q+1}$ & $\left(q^{2}+q+1\right) \mid k$ \\
18,19 & $k \in \mathbb{Z}_{q^{3}-1}$ & $(q-1),\left(q^{3}-1\right) / 2 \mid k$ \\
20 & $(k, l) \in \mathbb{Z}_{q^{3}-1} \times \mathbb{Z}_{q-1}$ & $\operatorname{many}$ \\
21,22 & $k \in \mathbb{Z}_{q+1}$ & $(q+1) / 2 \mid k$ \\
23 & $k \in \mathbb{Z}_{(q+1)\left(q^{3}-1\right)}$ & $(q+1),\left(q^{3}-1\right),(q+1)\left(q^{3}-1\right) / 2 \mid k$ \\
$24,25,26$ & $k \in \mathbb{Z}_{q^{2}-q+1}$ & $\left(q^{2}-q+1\right) \mid k$ \\
27,28 & $k \in \mathbb{Z}_{q^{3}+1}$ & $(q+1),\left(q^{3}+1\right) / 2 \mid k$ \\
29 & $k \in \mathbb{Z}_{(q-1)\left(q^{3}+1\right)}$ & $(q-1),\left(q^{3}+1\right),(q-1)\left(q^{3}+1\right) / 2 \mid k$ \\
30 & $(k, l) \in \mathbb{Z}_{q^{2}+q+1}$ & $\operatorname{many}$ \\
31 & $(k, l) \in \mathbb{Z}_{q^{2}-q+1}^{2}$ & $\operatorname{many}$ \\
32 & $k \in \mathbb{Z}_{q^{4}-q^{2}+1}$ & $\left(q^{4}-q^{2}+1\right) \mid k$ \\
33 & $(k, l) \in \mathbb{Z}_{q^{3}+1} \times \mathbb{Z}_{q+1}$ & many
\end{tabular}

Table (11.9 2-B). Some $S y l_{p}$-decompositions of irreducible characters for ${ }^{3} D_{4}(q)$ with $q$ odd.

\begin{tabular}{c|c||c|c||c|c||c|c}
$h$ & $\mathbf{v}$ & $h$ & $\mathbf{v}$ & $h$ & $\mathbf{v}$ & $h$ & $\mathbf{v}$ \\
\hline 10 & $(9,21)$ & 11 & $(9,27)$ & 12 & $(10,28)$ & 12 & $(11,22)$ \\
13 & $(9,10)$ & 14 & $(11,12)$ & 14 & $(13,29)$ & 17 & $(15,23)$ \\
18 & $(9,11)$ & 18 & $(15,16)$ & 19 & $(10,12)$ & 19 & $(16,17)$ \\
19 & $(18,23)$ & 20 & $(13,14)$ & 20 & $(18,19)$ & 22 & $(21,33)$ \\
23 & $(21,22)$ & 26 & $(25,28)$ & 27 & $(24,25)$ & 28 & $(27,33)$ \\
29 & $(24,26)$ & 29 & $(27,28)$ & 31 & $(25,25,25,33)$ & 8 & $(1,21,24,25,33)$ \\
8 & $(5,6,7,21,33)$ & 32 & $(5,5,5,5,25,33)$ & & & &
\end{tabular}

Table [11.9.2-C). The $S y l_{p}$-vanishing characters of degree $S t(1)$ for ${ }^{3} D_{4}(q)$ with $q$ odd.

\begin{tabular}{c|c||c|c||c|c||c|c}
$j$ & $\mathbf{v}$ & $j$ & $\mathbf{v}$ & $j$ & $\mathbf{v}$ & $j$ & $\mathbf{v}$ \\
\hline 1 & $(1,21,24,25,33)$ & 2 & $(5,6,7,21,33)$ & 3 & $(2,5,6,24,25,33)$ & 4 & $(1,21,27,33)$ \\
5 & $(1,21,28)$ & 6 & $(1,22,24,25)$ & 7 & $(1,22,27)$ & 8 & $(2,5,6,27,33)$ \\
9 & $(2,5,6,28)$ & 10 & $(5,6,7,22)$ & 11 & $(8)$ & &
\end{tabular}




\subsection{Groups $G_{2}(q)$.}

Table (11.10,1-A). CHEviE sets $\mathbf{X}_{h}$, their degrees and parameters for $G_{2}(q)$ with $q$ even and $q \equiv 1(\bmod 3)$.

\begin{tabular}{l|l||l|l}
$h$ & $\operatorname{deg} \chi_{h}$ & $h$ & $\operatorname{deg} \chi_{h}$ \\
\hline 1 & 1 & 2 & $q(q+1)^{2}\left(q^{2}+q+1\right) / 6$ \\
3 & $q(q+1)^{2}\left(q^{2}-q+1\right) / 2$ & 4,5 & $q\left(q^{2}+q+1\right)\left(q^{2}-q+1\right) / 3$ \\
6 & $q(q-1)^{2}\left(q^{2}-q+1\right) / 6$ & 7 & $q(q-1)^{2}\left(q^{2}+q+1\right) / 2$ \\
8,9 & $q(q-1)^{2}(q+1)^{2} / 3$ & 10 & $q^{6}$ \\
11 & $(q+1)\left(q^{2}-q+1\right)$ & 12 & $q(q+1)^{2}\left(q^{2}-q+1\right)$ \\
13 & $q^{3}(q+1)\left(q^{2}-q+1\right)$ & 14,16 & $\left(q^{2}+q+1\right)(q+1)\left(q^{2}-q+1\right)$ \\
15,17 & $q\left(q^{2}+q+1\right)(q+1)\left(q^{2}-q+1\right)$ & 18,20 & $\left(q^{2}-q+1\right)(q-1)\left(q^{2}+q+1\right)$ \\
19,21 & $q\left(q^{2}-q+1\right)(q-1)\left(q^{2}+q+1\right)$ & 22 & $(q+1)^{2}\left(q^{2}+q+1\right)\left(q^{2}-q+1\right)$ \\
23 & $(q-1)^{2}\left(q^{2}-q+1\right)\left(q^{2}+q+1\right)$ & 24,25 & $(q-1)(q+1)\left(q^{2}+q+1\right)\left(q^{2}-q+1\right)$ \\
26 & $(q-1)^{2}(q+1)^{2}\left(q^{2}-q+1\right)$ & 27 & $(q+1)^{2}(q-1)^{2}\left(q^{2}+q+1\right)$
\end{tabular}

\begin{tabular}{l|l|l}
$h$ & $I_{h}$ & exceptions \\
\hline $1, \ldots, 13$ & - & \\
14,15 & $k \in \mathbb{Z}_{q-1}$ & $(q-1) / 3 \mid k$ \\
16,17 & $k \in \mathbb{Z}_{q-1}$ & $(q-1) \mid k$ \\
$18, \ldots, 21$ & $k \in \mathbb{Z}_{q+1}$ & $(q+1) \mid k$ \\
22 & $(k, l) \in \mathbb{Z}_{q-1}^{2}$ & $(q-1) \mid k, l,(k \pm l),(2 k-l),(k-2 l)$ \\
23 & $(k, l) \in \mathbb{Z}_{q+1}^{2}$ & $(q+1) \mid k, l,(k \pm l),(2 k-l),(k-2 l)$ \\
24,25 & $k \in \mathbb{Z}_{q 2}-1$ & $(q \pm 1) \mid k$ \\
26 & $k \in \mathbb{Z}_{q^{2}+q+1}$ & $\left(q^{2}+q+1\right) / 3 \mid k$ \\
27 & $k \in \mathbb{Z}_{q^{2}-q+1}$ & $\left(q^{2}-q+1\right) \mid k$
\end{tabular}

Table (11.10,1-B). Some $S y l_{p}$-decompositions of irreducible characters for $G_{2}(q)$ with $q$ even and $q \equiv 1(\bmod 3)$.

\begin{tabular}{c|c||c|c||c|c||c|c||c|c}
$h$ & $\mathbf{v}$ & $h$ & $\mathbf{v}$ & $h$ & $\mathbf{v}$ & $h$ & $\mathbf{v}$ & $h$ & $\mathbf{v}$ \\
\hline 8 & $(9)$ & 13 & $(11,24)$ & 14 & $(11,12)$ & 15 & $(12,13)$ & 15 & $(14,24)$ \\
17 & $(16,25)$ & 19 & $(18,23)$ & 21 & $(20,23)$ & 22 & $(14,15)$ & 22 & $(16,17)$ \\
24 & $(18,19)$ & 25 & $(20,21)$ & 12 & $(2,3,9)$ & 10 & $(1,18,20,23)$ & 16 & $(1,2,3,5)$
\end{tabular}

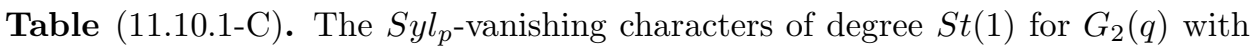
$q$ even and $q \equiv 1(\bmod 3)$.

\begin{tabular}{c|c||c|c||c|c||c|c}
$j$ & $\mathbf{v}$ & $j$ & $\mathbf{v}$ & $j$ & $\mathbf{v}$ & $j$ & $\mathbf{v}$ \\
\hline 1 & $(1,18,20,23)$ & 2 & $(1,18,21)$ & 3 & $(1,19,20)$ & 4 & $(4,6,7,18,23)$ \\
5 & $(4,6,7,19)$ & 6 & $(5,6,7,20,23)$ & 7 & $(5,6,7,21)$ & 8 & $(10)$
\end{tabular}

Table (11.10,2-A). CHEviE sets $\mathbf{X}_{h}$, their degrees and parameters for $G_{2}(q)$ with $q$ even and $q \equiv-1(\bmod 3)$.

\begin{tabular}{l|l||l|l}
$h$ & $\operatorname{deg} \chi_{h}$ & $h$ & $\operatorname{deg} \chi_{h}$ \\
\hline 1 & 1 & 2 & $q(q+1)^{2}\left(q^{2}+q+1\right) / 6$ \\
3 & $q(q+1)^{2}\left(q^{2}-q+1\right) / 2$ & 4,5 & $q\left(q^{2}+q+1\right)\left(q^{2}-q+1\right) / 3$ \\
6 & $q(q-1)^{2}\left(q^{2}-q+1\right) / 6$ & 7 & $q(q-1)^{2}\left(q^{2}+q+1\right) / 2$ \\
8,9 & $q(q-1)^{2}(q+1)^{2} / 3$ & 10 & $q^{6}$ \\
11 & $(q-1)\left(q^{2}+q+1\right)$ & 12 & $q(q-1)^{2}\left(q^{2}+q+1\right)$ \\
13 & $q^{3}(q-1)\left(q^{2}+q+1\right)$ & 14,16 & $\left(q^{2}+q+1\right)(q+1)\left(q^{2}-q+1\right)$ \\
15,17 & $q\left(q^{2}+q+1\right)(q+1)\left(q^{2}-q+1\right)$ & 18,20 & $\left(q^{2}-q+1\right)(q-1)\left(q^{2}+q+1\right)$ \\
19,21 & $q\left(q^{2}-q+1\right)(q-1)\left(q^{2}+q+1\right)$ & 22 & $(q+1)^{2}\left(q^{2}+q+1\right)\left(q^{2}-q+1\right)$ \\
23 & $(q-1)^{2}\left(q^{2}-q+1\right)\left(q^{2}+q+1\right)$ & 24,25 & $(q-1)(q+1)\left(q^{2}+q+1\right)\left(q^{2}-q+1\right)$ \\
26 & $(q-1)^{2}(q+1)^{2}\left(q^{2}-q+1\right)$ & 27 & $(q+1)^{2}(q-1)^{2}\left(q^{2}+q+1\right)$
\end{tabular}




\begin{tabular}{l|l|l}
$h$ & $I_{h}$ & exceptions \\
\hline $1, \ldots, 13$ & - & \\
$14, \ldots, 17$ & $k \in \mathbb{Z}_{q-1}$ & $(q-1) \mid k$ \\
18,19 & $k \in \mathbb{Z}_{q+1}$ & $(q+1) \mid k$ \\
20,21 & $k \in \mathbb{Z}_{q+1}$ & $(q+1) / 3 \mid k$ \\
22 & $(k, l) \in \mathbb{Z}_{q-1}^{2}$ & $(q-1) \mid k, l,(k \pm l),(2 k-l),(k-2 l)$ \\
23 & $(k, l) \in \mathbb{Z}_{q+1}^{2}$ & $(q+1) \mid k, l,(k \pm l),(2 k-l),(k-2 l)$ \\
24,25 & $k \in \mathbb{Z}_{q^{2}-1}$ & $(q \pm 1) \mid k$ \\
26 & $k \in \mathbb{Z}_{q^{2}+q+1}$ & $\left(q^{2}+q+1\right) \mid k$ \\
27 & $\mathbb{Z}_{q^{2}-q+1}$ & $\left(q^{2}-q+1\right) / 3 \mid k$
\end{tabular}

Table (11.10,2-B). Some $S y l_{p}$-decompositions of irreducible characters for $G_{2}(q)$ with $q$ even and $q \equiv-1(\bmod 3)$.

\begin{tabular}{c|c||c|c||c|c||c|c||c|c}
$h$ & $\mathbf{v}$ & $h$ & $\mathbf{v}$ & $h$ & $\mathbf{v}$ & $h$ & $\mathbf{v}$ & $h$ & $\mathbf{v}$ \\
\hline 8 & $(9)$ & 13 & $(12,21)$ & 15 & $(14,24)$ & 17 & $(16,25)$ & 19 & $(18,23)$ \\
20 & $(11,12)$ & 21 & $(20,23)$ & 22 & $(14,15)$ & 22 & $(16,17)$ & 24 & $(18,19)$ \\
25 & $(11,13)$ & 25 & $(20,21)$ & 4 & $(1,9,11)$ & 12 & $(6,7,9)$ & 16 & $(1,2,3,5)$ \\
$h$ & \multicolumn{2}{|c|}{$\mathbf{v}$} & $h$ & \multicolumn{2}{|c|}{$\mathbf{v}$} & $h$ & \multicolumn{2}{|c|}{$\mathbf{v}$} \\
\hline 14 & $(1,1,2,3,9,11)$ & 10 & $(1,6,7,9,11,18,23)$ & 27 & $(6,6,6,7,7,7,9,9,9,23)$
\end{tabular}

Table $111.10,2-\mathrm{C})$. The $S y l_{p}$-vanishing characters of degree $S t(1)$ for $G_{2}(q)$ with $q$ even and $q \equiv-1(\bmod 3)$.

\begin{tabular}{c|c||c|c||c|c}
$j$ & $\mathbf{v}$ & $j$ & $\mathbf{v}$ & $j$ & $\mathbf{v}$ \\
\hline 1 & $(1,6,7,8,11,18,23)$ & 2 & $(1,6,7,8,11,19)$ & 3 & $(1,6,7,9,11,18,23)$ \\
4 & $(1,6,7,9,11,19)$ & 5 & $(1,11,12,18,23)$ & 6 & $(1,11,12,19)$ \\
7 & $(1,18,20,23)$ & 8 & $(1,18,21)$ & 9 & $(1,19,20)$ \\
10 & $(4,6,7,18,23)$ & 11 & $(4,6,7,19)$ & 12 & $(5,6,6,7,7,8,11,23)$ \\
13 & $(5,6,6,7,7,9,11,23)$ & 14 & $(5,6,7,11,12,23)$ & 15 & $(5,6,7,20,23)$ \\
16 & $(5,6,7,21)$ & 17 & $(10)$ & &
\end{tabular}

Table (11.10,3-A). CHEvIE sets $\mathbf{X}_{h}$, their degrees and parameters for $G_{2}(q)$ with $q$ odd and $q \equiv 0(\bmod 3)$.

\begin{tabular}{l|l||l|l}
$h$ & $\operatorname{deg} \chi_{h}$ & $h$ & $\operatorname{deg} \chi_{h}$ \\
\hline 1 & 1 & 2 & $q(q+1)^{2}\left(q^{2}+q+1\right) / 6$ \\
3 & $q(q+1)^{2}\left(q^{2}-q+1\right) / 2$ & 4,5 & $q\left(q^{2}+q+1\right)\left(q^{2}-q+1\right) / 3$ \\
6 & $q(q-1)^{2}\left(q^{2}-q+1\right) / 6$ & 7 & $q(q-1)^{2}\left(q^{2}+q+1\right) / 2$ \\
8,9 & $q(q-1)^{2}(q+1)^{2} / 3$ & 10 & $q^{6}$ \\
11 & $\left(q^{2}+q+1\right)\left(q^{2}-q+1\right)$ & 12,13 & $q\left(q^{2}+q+1\right)\left(q^{2}-q+1\right)$ \\
14 & $q^{2}\left(q^{2}+q+1\right)\left(q^{2}-q+1\right)$ & 15,17 & $\left(q^{2}+q+1\right)(q+1)\left(q^{2}-q+1\right)$ \\
16,18 & $q\left(q^{2}+q+1\right)(q+1)\left(q^{2}-q+1\right)$ & 19,21 & $\left(q^{2}-q+1\right)(q-1)\left(q^{2}+q+1\right)$ \\
20,22 & $q\left(q^{2}-q+1\right)(q-1)\left(q^{2}+q+1\right)$ & 23 & $(q+1)^{2}\left(q^{2}+q+1\right)\left(q^{2}-q+1\right)$ \\
24 & $(q-1)^{2}\left(q^{2}-q+1\right)\left(q^{2}+q+1\right)$ & 25,26 & $(q-1)(q+1)\left(q^{2}+q+1\right)\left(q^{2}-q+1\right)$ \\
27 & $(q-1)^{2}(q+1)^{2}\left(q^{2}-q+1\right)$ & 28 & $(q+1)^{2}(q-1)^{2}\left(q^{2}+q+1\right)$
\end{tabular}

\begin{tabular}{l|l|l}
$h$ & $I_{h}$ & exceptions \\
\hline $1, \ldots 14$ & - & \\
$15, \ldots, 18$ & $k \in \mathbb{Z}_{q-1}$ & $(q-1) / 2 \mid k$ \\
$19, \ldots, 22$ & $k \in \mathbb{Z}_{q+1}$ & $(q+1) / 2 \mid k$ \\
23 & $(k, l) \in \mathbb{Z}_{q-1}^{2}$ & $(q-1) \mid k, l,(k \pm l),(2 k-l),(k-2 l)$ \\
24 & $(k, l) \in \mathbb{Z}_{q+1}^{2}$ & $(q+1) \mid k, l,(k \pm l),(2 k-l),(k-2 l)$ \\
25,26 & $k \in \mathbb{Z}_{q^{2}-1}$ & $(q \pm 1) \mid k$ \\
27 & $k \in \mathbb{Z}_{q^{2}+q+1}$ & $\left(q^{2}+q+1\right) \mid k$ \\
28 & $k \in \mathbb{Z}_{q^{2}-q+1}$ & $\left(q^{2}-q+1\right) \mid k$
\end{tabular}


Table (11.10,3-B). Some $S y l_{p}$-decompositions of irreducible characters for $G_{2}(q)$ with $q$ odd and $q \equiv 0(\bmod 3)$.

\begin{tabular}{c|c||c|c||c|c||c|c||c|c}
$h$ & $\mathbf{v}$ & $h$ & $\mathbf{v}$ & $h$ & $\mathbf{v}$ & $h$ & $\mathbf{v}$ & $h$ & $\mathbf{v}$ \\
\hline 12 & $(11,19)$ & 13 & $(11,21)$ & 14 & $(12,22)$ & 14 & $(13,20)$ & 15 & $(11,13)$ \\
16 & $(12,14)$ & 16 & $(15,25)$ & 17 & $(11,12)$ & 18 & $(13,14)$ & 18 & $(17,26)$ \\
20 & $(19,24)$ & 22 & $(21,24)$ & 23 & $(15,16)$ & 23 & $(17,18)$ & 25 & $(19,20)$ \\
26 & $(21,22)$ & 10 & $(1,19,21,24)$ & & & & & &
\end{tabular}

Table (11.10,3-C). The $S y l_{p}$-vanishing characters of degree $\operatorname{St}(1)$ for $G_{2}(q)$ with $q$ odd and $q \equiv 0(\bmod 3)$.

\begin{tabular}{c|c||c|c||c|c||c|c}
$j$ & $\mathbf{v}$ & $j$ & $\mathbf{v}$ & $j$ & $\mathbf{v}$ & $j$ & $\mathbf{v}$ \\
\hline 1 & $(1,19,21,24)$ & 2 & $(1,19,22)$ & 3 & $(1,20,21)$ & 4 & $(4,6,7,19,24)$ \\
5 & $(4,6,7,20)$ & 6 & $(5,6,7,21,24)$ & 7 & $(5,6,7,22)$ & 8 & $(10)$
\end{tabular}

Table (11.10,4-A). CHEVIE sets $\mathbf{X}_{h}$, their degrees and parameters for $G_{2}(q)$ with $q$ odd and $q \equiv 1(\bmod 3)$.

\begin{tabular}{l|l||l|l}
$h$ & $\operatorname{deg} \chi_{h}$ & $h$ & $\operatorname{deg} \chi_{h}$ \\
\hline 1 & 1 & 2 & $q(q+1)^{2}\left(q^{2}+q+1\right) / 6$ \\
3 & $q(q+1)^{2}\left(q^{2}-q+1\right) / 2$ & 4,5 & $q\left(q^{2}+q+1\right)\left(q^{2}-q+1\right) / 3$ \\
6 & $q(q-1)^{2}\left(q^{2}-q+1\right) / 6$ & 7 & $q(q-1)^{2}\left(q^{2}+q+1\right) / 2$ \\
8,9 & $q(q-1)^{2}(q+1)^{2} / 3$ & 10 & $q^{6}$ \\
11 & $\left(q^{2}+q+1\right)\left(q^{2}-q+1\right)$ & 12,13 & $q\left(q^{2}+q+1\right)\left(q^{2}-q+1\right)$ \\
14 & $q^{2}\left(q^{2}+q+1\right)\left(q^{2}-q+1\right)$ & 15 & $(q+1)\left(q^{2}-q+1\right)$ \\
16 & $q(q+1)^{2}\left(q^{2}-q+1\right)$ & 17 & $q^{3}(q+1)\left(q^{2}-q+1\right)$ \\
18,20 & $\left(q^{2}+q+1\right)(q+1)\left(q^{2}-q+1\right)$ & 19,21 & $q\left(q^{2}+q+1\right)(q+1)\left(q^{2}-q+1\right)$ \\
22,24 & $\left(q^{2}-q+1\right)(q-1)\left(q^{2}+q+1\right)$ & 23,25 & $q\left(q^{2}-q+1\right)(q-1)\left(q^{2}+q+1\right)$ \\
26 & $(q+1)^{2}\left(q^{2}+q+1\right)\left(q^{2}-q+1\right)$ & 27 & $(q-1)^{2}\left(q^{2}-q+1\right)\left(q^{2}+q+1\right)$ \\
28,29 & $(q-1)(q+1)\left(q^{2}+q+1\right)\left(q^{2}-q+1\right)$ & 30 & $(q-1)^{2}(q+1)^{2}\left(q^{2}-q+1\right)$ \\
31 & $(q+1)^{2}(q-1)^{2}\left(q^{2}+q+1\right)$ & &
\end{tabular}

\begin{tabular}{l|l|l}
$h$ & $I_{h}$ & exceptions \\
\hline $1, \ldots, 17$ & - & \\
18,19 & $k \in \mathbb{Z}_{q-1}$ & $(q-1) / 3,(q-1) / 2 \mid k$ \\
20,21 & $k \in \mathbb{Z}_{q-1}$ & $(q-1) / 2 \mid k$ \\
$22, \ldots, 25$ & $k \in \mathbb{Z}_{q+1}$ & $(q+1) / 2 \mid k$ \\
26 & $(k, l) \in \mathbb{Z}_{q-1}^{2}$ & $(q-1) \mid k, l,(k \pm l),(2 k-l),(k-2 l)$ \\
27 & $(k, l) \in \mathbb{Z}_{q+1}^{2}$ & $(q+1) \mid k, l,(k \pm l),(2 k-l),(k-2 l)$ \\
28,29 & $k \in \mathbb{Z}_{q^{2}-1}$ & $(q \pm 1) \mid k$ \\
30 & $k \in \mathbb{Z}_{q^{2}+q+1}$ & $\left(q^{2}+q+1\right) / 3 \mid k$ \\
31 & $k \in \mathbb{Z}_{q^{2}-q+1}$ & $\left(q^{2}-q+1\right) \mid k$
\end{tabular}

Table (11.10,4-B). Some $S y l_{p}$-decompositions of irreducible characters for $G_{2}(q)$ with $q$ odd and $q \equiv 1(\bmod 3)$.

\begin{tabular}{c|c||c|c||c|c||c|c||c|c}
$h$ & $\mathbf{v}$ & $h$ & $\mathbf{v}$ & $h$ & $\mathbf{v}$ & $h$ & $\mathbf{v}$ & $h$ & $\mathbf{v}$ \\
\hline 8 & $(9)$ & 12 & $(11,22)$ & 13 & $(11,24)$ & 14 & $(12,25)$ & 14 & $(13,23)$ \\
17 & $(15,28)$ & 18 & $(11,13)$ & 18 & $(15,16)$ & 19 & $(12,14)$ & 19 & $(16,17)$ \\
19 & $(18,28)$ & 20 & $(11,12)$ & 21 & $(13,14)$ & 21 & $(20,29)$ & 23 & $(22,27)$ \\
25 & $(24,27)$ & 26 & $(18,19)$ & 26 & $(20,21)$ & 28 & $(22,23)$ & 29 & $(24,25)$ \\
16 & $(2,3,9)$ & 10 & $(1,22,24,27)$ & & & & & &
\end{tabular}

Table 11.10,4-C). The $S y l_{p}$-vanishing characters of degree $S t(1)$ for $G_{2}(q)$ with $q$ odd and $q \equiv 1(\bmod 3)$.

\begin{tabular}{c|c||c|c||c|c||c|c}
$j$ & $\mathbf{v}$ & $j$ & $\mathbf{v}$ & $j$ & $\mathbf{v}$ & $j$ & $\mathbf{v}$ \\
\hline 1 & $(1,22,24,27)$ & 2 & $(1,22,25)$ & 3 & $(1,23,24)$ & 4 & $(4,6,7,22,27)$ \\
5 & $(4,6,7,23)$ & 6 & $(5,6,7,24,27)$ & 7 & $(5,6,7,25)$ & 8 & $(10)$
\end{tabular}


Table (11.10, 5-A). CHEvIE sets $\mathbf{X}_{h}$, their degrees and parameters for $G_{2}(q)$ with $q$ odd and $q \equiv-1(\bmod 3)$.

\begin{tabular}{l|l||l|l}
$h$ & $\operatorname{deg} \chi_{h}$ & $h$ & $\operatorname{deg} \chi_{h}$ \\
\hline 1 & 1 & 2 & $q(q+1)^{2}\left(q^{2}+q+1\right) / 6$ \\
3 & $q(q+1)^{2}\left(q^{2}-q+1\right) / 2$ & 4,5 & $q\left(q^{2}+q+1\right)\left(q^{2}-q+1\right) / 3$ \\
6 & $q(q-1)^{2}\left(q^{2}-q+1\right) / 6$ & 7 & $q(q-1)^{2}\left(q^{2}+q+1\right) / 2$ \\
8,9 & $q(q-1)^{2}(q+1)^{2} / 3$ & 10 & $q^{6}$ \\
11 & $\left(q^{2}+q+1\right)\left(q^{2}-q+1\right)$ & 12,13 & $q\left(q^{2}+q+1\right)\left(q^{2}-q+1\right)$ \\
14 & $q^{2}\left(q^{2}+q+1\right)\left(q^{2}-q+1\right)$ & 15 & $(q-1)\left(q^{2}+q+1\right)$ \\
16 & $q(q-1)^{2}\left(q^{2}+q+1\right)$ & 17 & $q^{3}(q-1)\left(q^{2}+q+1\right)$ \\
18,20 & $\left(q^{2}+q+1\right)(q+1)\left(q^{2}-q+1\right)$ & 19,21 & $q\left(q^{2}+q+1\right)(q+1)\left(q^{2}-q+1\right)$ \\
22,24 & $\left(q^{2}-q+1\right)(q-1)\left(q^{2}+q+1\right)$ & 23,25 & $q\left(q^{2}-q+1\right)(q-1)\left(q^{2}+q+1\right)$ \\
26 & $(q+1)^{2}\left(q^{2}+q+1\right)\left(q^{2}-q+1\right)$ & 27 & $(q-1)^{2}\left(q^{2}-q+1\right)\left(q^{2}+q+1\right)$ \\
28,29 & $(q-1)(q+1)\left(q^{2}+q+1\right)\left(q^{2}-q+1\right)$ & 30 & $(q-1)^{2}(q+1)^{2}\left(q^{2}-q+1\right)$ \\
31 & $(q+1)^{2}(q-1)^{2}\left(q^{2}+q+1\right)$ & &
\end{tabular}

\begin{tabular}{l|l|l}
$h$ & $I_{h}$ & exceptions \\
\hline $1, \ldots, 17$ & - & \\
$18, \ldots, 21$ & $k \in \mathbb{Z}_{q-1}$ & $(q-1) / 2 \mid k$ \\
22,23 & $k \in \mathbb{Z}_{q+1}$ & $(q+1) / 2 \mid k$ \\
24,25 & $k \in \mathbb{Z}_{q+1}$ & $(q+1) / 2,(q+1) / 3 \mid k$ \\
26 & $(k, l) \in \mathbb{Z}_{q-1}^{2}$ & $(q-1) \mid k, l,(k \pm l),(2 k-l),(k-2 l)$ \\
27 & $(k, l) \in \mathbb{Z}_{q+1}^{2}$ & $(q+1) \mid k, l,(k \pm l),(2 k-l),(k-2 l)$ \\
28,29 & $k \in \mathbb{Z}_{q^{2}-1}$ & $(q \pm 1) \mid k$ \\
30 & $k \in \mathbb{Z}_{q^{2}+q+1}$ & $\left(q^{2}+q+1\right) \mid k$ \\
31 & $k \in \mathbb{Z}_{q^{2}-q+1}$ & $\left(q^{2}-q+1\right) / 3 \mid k$
\end{tabular}

Table (11.10, 5 -B). Some $S y l_{p}$-decompositions of irreducible characters for $G_{2}(q)$ with $q$ odd and $q \equiv-1(\bmod 3)$.

\begin{tabular}{c|c|c||c|c||c|c||c|c||c|c} 
& $h$ & $\mathbf{v}$ & $h$ & $\mathbf{v}$ & $h$ & $\mathbf{v}$ & $h$ & $\mathbf{v}$ & $h$ & $\mathbf{v}$ \\
\hline 8 & $(9)$ & 12 & $(11,22)$ & 13 & $(11,24)$ & 14 & $(12,25)$ & 14 & $(13,23)$ \\
& $(16,25)$ & 18 & $(11,13)$ & 19 & $(12,14)$ & 19 & $(18,28)$ & 20 & $(11,12)$ \\
& 21 & $(13,14)$ & 21 & $(20,29)$ & 23 & $(22,27)$ & 24 & $(15,16)$ & 25 & $(24,27)$ \\
& 26 & $(18,19)$ & 26 & $(20,21)$ & 28 & $(22,23)$ & 29 & $(15,17)$ & 29 & $(24,25)$ \\
$h$ & $\mathbf{v}$ & $\mathbf{v}$ & $h$ & $\mathbf{v}$ & $h$ & \multicolumn{1}{|c||}{$\mathbf{v}$} & & $h$ & \\
\hline 4 & $(1,9,15)$ & 16 & $(6,7,9)$ & 10 & $(1,6,7,9,15,22,27)$ & 31 & $(6,6,6,7,7,7,9,9,9,27)$ \\
\end{tabular}


Table (11.10, 5 -C). The $S y l_{p}$-vanishing characters of degree $S t(1)$ for $G_{2}(q)$ with $q$ odd and $q \equiv-1(\bmod 3)$.

\begin{tabular}{c|c||c|c||c|c}
$j$ & $\mathbf{v}$ & $j$ & $\mathbf{v}$ & $j$ & $\mathbf{v}$ \\
\hline 1 & $(5,6,6,7,7,9,15,27)$ & 2 & $(1,6,7,9,15,22,27)$ & 3 & $(1,6,7,8,15,22,27)$ \\
4 & $(1,6,7,8,15,23)$ & 5 & $(1,6,7,9,15,23)$ & 6 & $(1,15,16,22,27)$ \\
7 & $(1,15,16,23)$ & 8 & $(1,22,24,27)$ & 9 & $(1,22,25)$ \\
10 & $(1,23,24)$ & 11 & $(4,6,7,22,27)$ & 12 & $(4,6,7,23)$ \\
13 & $(5,6,6,7,7,8,15,27)$ & 14 & $(5,6,7,15,16,27)$ & 15 & $(5,6,7,24,27)$ \\
16 & $(5,6,7,25)$ & 17 & $(10)$ & &
\end{tabular}

11.11. Groups ${ }^{2} F_{4}\left(q^{2}\right)$.

Table (11.11 A). Some CHEVIE sets $\mathbf{X}_{h}$, their degrees and parameters for ${ }^{2} F_{4}\left(q^{2}\right)$.

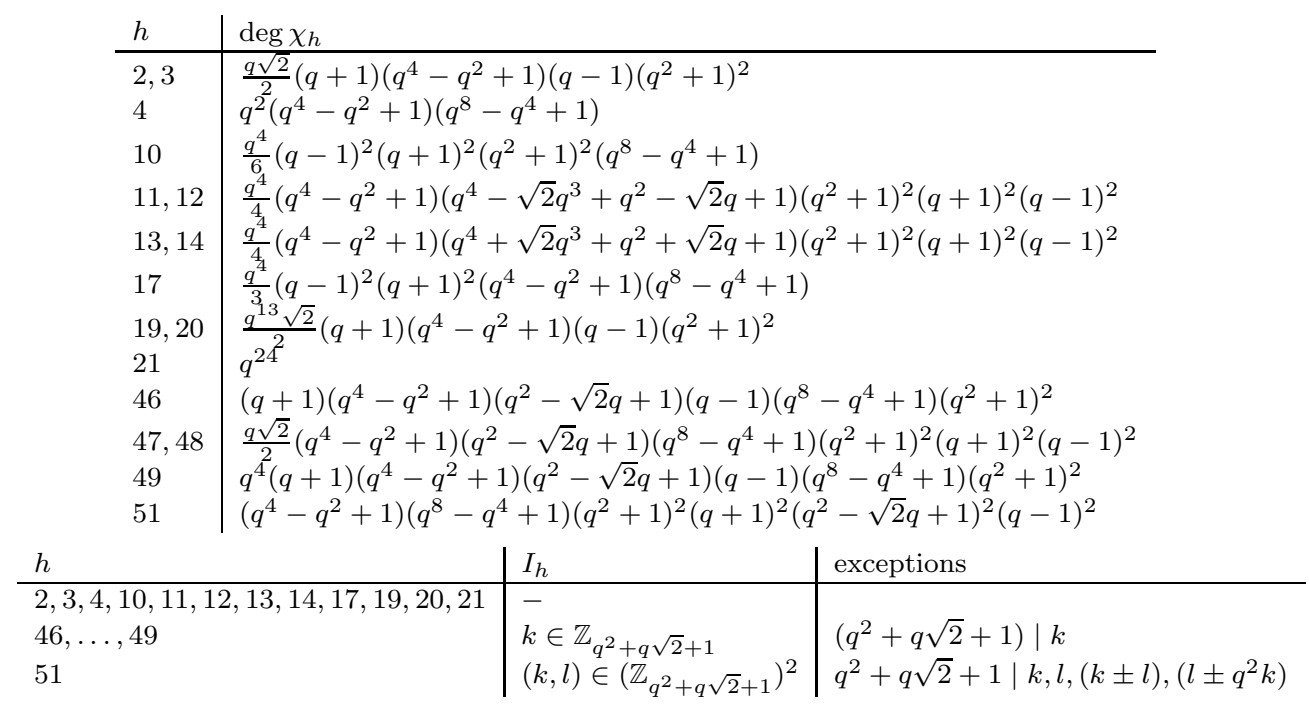

Table (11.11-B). Some $S y l_{p}$-decompositions of irreducible characters for ${ }^{2} F_{4}\left(q^{2}\right)$.

\begin{tabular}{c|c||c|c||c|c||c|c}
$h$ & $\mathbf{v}$ & $h$ & $\mathbf{v}$ & $h$ & $\mathbf{v}$ & $h$ & $\mathbf{v}$ \\
\hline 15 & $(16)$ & 23 & $(2,19)$ & 24 & $(3,20)$ & 27 & $(26,34)$ \\
28 & $(22,25)$ & 28 & $(26,27)$ & 31 & $(30,33)$ & 32 & $(29,30)$ \\
33 & $(32,38)$ & 34 & $(29,31)$ & 34 & $(32,33)$ & 35 & $(42,45)$ \\
36 & $(46,49)$ & 39 & $(30,30,30,38)$ & 45 & $(37,42,43,44)$ & 49 & $(46,47,48,51)$ \\
22 & $(1,4,5,6,7)$ & 25 & $(5,6,7,18,21)$ & 26 & $(1,5,6,7,18)$ & 37 & $(47,47,48,48,51)$ \\
50 & $(37,43,43,44,44)$ & & & & &
\end{tabular}

Table (11.11 C). The $S y l_{p}$-vanishing characters of degree $S t(1)$ for ${ }^{2} F_{4}\left(q^{2}\right)$.

\begin{tabular}{c|c||c|c}
$j$ & $\mathbf{v}$ & $j$ & $\mathbf{v}$ \\
\hline 1 & $(2,4,10,11,13,17,20,46,47,47,48,51)$ & 2 & $(3,4,10,12,14,17,19,46,47,48,48,51)$ \\
3 & $(2,4,10,11,13,17,20,47,49)$ & 4 & $(3,4,10,12,14,17,19,48,49)$ \\
5 & $(21)$ & &
\end{tabular}




\subsection{Groups $G L(4, q)$.}

Table (11.12 A). CHEviE sets $\mathbf{X}_{h}$, their degrees and parameters for $G L(4, q)$.

\begin{tabular}{l|l||l|l}
$h$ & $\operatorname{deg} \chi_{h}$ & $h$ & $\operatorname{deg} \chi_{h}$ \\
\hline 1 & $q^{6}$ & 2 & $q^{3}\left(q^{2}+q+1\right)$ \\
3 & $q^{2}\left(q^{2}+1\right)$ & 4 & $q\left(q^{2}+q+1\right)$ \\
5 & 1 & 6 & $(q+1)\left(q^{2}+1\right) q^{3}$ \\
7 & $q\left(q^{2}+1\right)(q+1)^{2}$ & 8 & $\left(q^{2}+1\right)(q+1)$ \\
9 & $q^{2}\left(q^{2}+1\right)\left(q^{2}+q+1\right)$ & 10 & $q\left(q^{2}+1\right)\left(q^{2}+q+1\right)$ \\
11 & $q\left(q^{2}+1\right)\left(q^{2}+q+1\right)$ & 12 & $\left(q^{2}+1\right)\left(q^{2}+q+1\right)$ \\
13 & $q^{2}\left(q^{2}+q+1\right)(q-1)^{2}$ & 14 & $\left(q^{2}+q+1\right)(q-1)^{2}$ \\
15 & $q(q+1)\left(q^{2}+q+1\right)\left(q^{2}+1\right)$ & 16 & $(q+1)\left(q^{2}+1\right)\left(q^{2}+q+1\right)$ \\
17 & $q(q-1)\left(q^{2}+q+1\right)\left(q^{2}+1\right)$ & 18 & $(q-1)\left(q^{2}+q+1\right)\left(q^{2}+1\right)$ \\
19 & $\left(q^{2}+1\right)\left(q^{2}+q+1\right)(q+1)^{2}$ & 20 & $(q-1)(q+1)\left(q^{2}+q+1\right)\left(q^{2}+1\right)$ \\
21 & $\left(q^{2}+1\right)\left(q^{2}+q+1\right)(q-1)^{2}$ & 22 & $\left(q^{2}+1\right)(q-1)^{2}(q+1)^{2}$ \\
23 & $(q+1)\left(q^{2}+q+1\right)(q-1)^{3}$ & \multicolumn{2}{|l}{}
\end{tabular}

\begin{tabular}{l|l|l}
$h$ & $I_{h}$ & exceptions \\
\hline $1 \ldots, 5$ & $k_{1} \in \mathbb{Z}_{q-1}$ & \\
$6, \ldots, 12$ & $\left(k_{1}, k_{2}\right) \in \mathbb{Z}_{q-1} \times \mathbb{Z}_{q-1}$ & $(q-1) \mid\left(k_{1}-k_{2}\right)$ \\
13,14 & $k_{1} \in \mathbb{Z}_{q^{2}-1}$ & $(q+1) \mid k_{1}$ \\
15,16 & $\left(k_{1}, k_{2}, k_{3}\right) \in \mathbb{Z}_{q-1}^{3}$ & $(q-1) \mid\left(k_{i}-k_{j}\right), i \neq j$ \\
17,18 & $\left(k_{1}, k_{2}\right) \in \mathbb{Z}_{q-1} \times \mathbb{Z}_{q^{2}-1}$ & $\left(q^{2}-1\right) \mid(q+1) k_{1}-k_{2}$ \\
& $\left(k_{1}, k_{2}, k_{3}, k_{4}\right) \in \mathbb{Z}_{q-1}^{4}$ & $(q) \mid k_{2}$ \\
19 & $\left(k_{1}, k_{2}, k_{3}\right) \in \mathbb{Z}_{q^{2}-1} \times \mathbb{Z}_{q-1} \times \mathbb{Z}_{q-1}$ & $(q-1) \mid\left(k_{i}-k_{j}\right), i \neq j$ \\
20 & & $(q+1) \mid k_{1}$ \\
& & $(q-1) \mid\left(k_{2}-k_{3}\right)$ \\
& $\left(k_{1}, k_{2}\right) \in \mathbb{Z}_{q^{2}-1} \times \mathbb{Z}_{q^{2}-1}$ & $(q+1) \mid k_{i}$ \\
& & $\left(q^{2}-1\right) \mid q k_{1}-k_{2}$ \\
22 & $\left(k_{1}, k_{2}\right) \in \mathbb{Z}_{q^{3}-1} \times \mathbb{Z}_{q-1}$ & $\left(q^{2}-1\right) \mid\left(k_{1}-k_{2}\right)$ \\
23 & $k_{1} \in \mathbb{Z}_{q^{4}-1}$ & $\left(q^{2}+q+1\right) \mid(q+1) k_{1}$ \\
& & $\left(q^{3}+q+q^{2}+1\right) \mid\left(q^{2}+q+1\right) k_{1}$ \\
& & $\left(q^{2}+1\right) \mid k_{1}$
\end{tabular}

Table (11.12-B). Some $S y l_{p}$-decompositions of irreducible characters for $G L(4, q)$.

\begin{tabular}{c|c||c|c||c|c||c|c||c|c}
$h$ & $\mathbf{v}$ & $h$ & $\mathbf{v}$ & $h$ & $\mathbf{v}$ & $h$ & $\mathbf{v}$ & $h$ & $\mathbf{v}$ \\
\hline 10 & $(11)$ & 6 & $(1,2)$ & 6 & $(8,20)$ & 7 & $(3,11)$ & 8 & $(4,5)$ \\
9 & $(3,6)$ & 9 & $(11,17)$ & 9 & $(12,20)$ & 11 & $(2,4)$ & 11 & $(12,18)$ \\
12 & $(3,8)$ & 13 & $(14,23)$ & 15 & $(6,7)$ & 15 & $(9,11)$ & 15 & $(16,20)$ \\
16 & $(7,8)$ & 16 & $(11,12)$ & 17 & $(18,21)$ & 19 & $(15,16)$ & 20 & $(17,18)$ \\
21 & $(13,14)$ & 2 & $(3,5,18)$ & 2 & $(4,14,18)$ & 1 & $(5,14,18,23)$ & &
\end{tabular}

Table (11.12 $\mathrm{C})$. The $S y l_{p}$-vanishing characters of degree $S t(1)$ for $G L(4, q)$.

\begin{tabular}{c|c||c|c||c|c}
$j$ & $\mathbf{v}$ & $j$ & $\mathbf{v}$ & $j$ & $\mathbf{v}$ \\
\hline 1 & $(5,14,18,23)$ & 2 & $(5,13,18)$ & 3 & $(1)$
\end{tabular}

Table (11.12-D). The $p$-vanishing characters of degree $S t(1)$ and their values for $G L(4, q)$ with $3 \mid(q+1)$.

In view of Lemma 9.1 we have the character $\psi=1_{G}+\chi_{14}(a)+\chi_{18}(0, a)+$ $\chi_{23}\left(\left(q^{2}-1\right) b\right)$. This character differs from $S t$ only on the following classes:

\begin{tabular}{l||l|l}
$C_{h}$ & $S t$ & $\psi$ \\
\hline$C_{13}\left(i_{1}\right)$ & $q^{2}$ & $3 q^{2}+2 \operatorname{Re}\left(\xi_{1}^{i_{1} a}\right)-2\left(q^{2}+1\right) \operatorname{Re}\left(\xi_{1}^{i_{1} a}\right)$ \\
$C_{17}\left(i_{1}, i_{2}\right)$ & $-q$ & $q-2(q-1) \operatorname{Re}\left(\xi_{1}^{i_{2} a}\right)-2 \operatorname{Re}\left(\xi_{1}^{i_{2} a}\right)$ \\
$C_{20}\left(i_{1}, i_{2}, i_{3}\right)$ & -1 & $1-2 \operatorname{Re}\left(\xi_{1}^{i_{1} a}\right)$ \\
$C_{21}\left(i_{1}, i_{2}\right)$ & 1 & $1+2\left(\operatorname{Re}\left(\xi_{1}^{\left(i_{1}+i_{2}\right) a}\right)+\operatorname{Re}\left(\xi_{1}^{\left(i_{1}-i_{2}\right) a}\right)-\operatorname{Re}\left(\xi_{1}^{i_{1} a}\right)-\operatorname{Re}\left(\xi_{1}^{i_{2} a}\right)\right)$ \\
$C_{23}\left(i_{1}\right)$ & -1 & $1+2\left(\operatorname{Re}\left(\xi^{i_{1} a}\right)-\operatorname{Re}\left(\xi_{2}^{i_{1} b}\right)-\operatorname{Re}\left(\xi_{2}^{i_{1} b}\right)\right)$
\end{tabular}




\subsection{Groups $G L(5, q)$.}

Table (11.13 A). CHEVIE sets $\mathbf{X}_{h}$, their degrees and parameters for $G L(5, q)$.

\begin{tabular}{|c|c|}
\hline$h$ & $\operatorname{deg} \chi_{h}$ \\
\hline 1 & $q^{10}$ \\
\hline 2 & $q^{6}(q+1)\left(q^{2}+1\right)$ \\
\hline 3 & $\left(q^{4}+q^{3}+q^{2}+q+1\right) q^{4}$ \\
\hline 4 & $q^{3}\left(q^{2}+1\right)\left(q^{2}+q+1\right)$ \\
\hline 5 & $q^{2}\left(q^{4}+q^{3}+q^{2}+q+1\right)$ \\
\hline 6 & $q(q+1)\left(q^{2}+1\right)$ \\
\hline 7 & 1 \\
\hline 8 & $q^{6}\left(q^{4}+q^{3}+q^{2}+q+1\right)$ \\
\hline 9 & $q^{3}\left(q^{2}+q+1\right)\left(q^{4}+q^{3}+q^{2}+q+1\right)$ \\
\hline 10 & $q^{2}\left(q^{2}+1\right)\left(q^{4}+q^{3}+q^{2}+q+1\right)$ \\
\hline 11 & $q\left(q^{2}+q+1\right)\left(q^{4}+q^{3}+q^{2}+q+1\right)$ \\
\hline 12 & $q^{4}+q^{3}+q^{2}+q+1$ \\
\hline 13 & $q^{4}\left(q^{2}+1\right)\left(q^{4}+q^{3}+q^{2}+q+1\right)$ \\
\hline 14 & $q^{3}\left(q^{2}+1\right)\left(q^{4}+q^{3}+q^{2}+q+1\right)$ \\
\hline 15 & $q^{2}(q+1)\left(q^{2}+1\right)\left(q^{4}+q^{3}+q^{2}+q+1\right)$ \\
\hline 16 & $q(q+1)\left(q^{2}+1\right)\left(q^{4}+q^{3}+q^{2}+q+1\right)$ \\
\hline 17 & $q\left(q^{2}+1\right)\left(q^{4}+q^{3}+q^{2}+q+1\right)$ \\
\hline 18 & $\left(q^{2}+1\right)\left(q^{4}+q^{3}+q^{2}+q+1\right)$ \\
\hline 19 & $q^{3}(q+1)\left(q^{2}+1\right)\left(q^{4}+q^{3}+q^{2}+q+1\right)$ \\
\hline 20 & $q\left(q^{2}+1\right)\left(q^{4}+q^{3}+q^{2}+q+1\right)(q+1)^{2}$ \\
\hline 21 & $(q+1)\left(q^{2}+1\right)\left(q^{4}+q^{3}+q^{2}+q+1\right)$ \\
\hline 22 & $q^{3}(q-1)\left(q^{2}+1\right)\left(q^{4}+q^{3}+q^{2}+q+1\right)$ \\
\hline 23 & $q(q-1)(q+1)\left(q^{2}+1\right)\left(q^{4}+q^{3}+q^{2}+q+1\right)$ \\
\hline 24 & $(q-1)\left(q^{2}+1\right)\left(q^{4}+q^{3}+q^{2}+q+1\right)$ \\
\hline 25 & $q^{2}\left(q^{2}+q+1\right)\left(q^{4}+q^{3}+q^{2}+q+1\right)\left(q^{2}+1\right)$ \\
\hline 26 & $q\left(q^{2}+q+1\right)\left(q^{4}+q^{3}+q^{2}+q+1\right)\left(q^{2}+1\right)$ \\
\hline 27 & $q\left(q^{2}+q+1\right)\left(q^{4}+q^{3}+q^{2}+q+1\right)\left(q^{2}+1\right)$ \\
\hline 28 & $\left(q^{2}+q+1\right)\left(q^{4}+q^{3}+q^{2}+q+1\right)\left(q^{2}+1\right)$ \\
\hline 29 & $q^{2}\left(q^{2}+q+1\right)\left(q^{4}+q^{3}+q^{2}+q+1\right)(q-1)^{2}$ \\
\hline 30 & $\left(q^{2}+q+1\right)\left(q^{4}+q^{3}+q^{2}+q+1\right)(q-1)^{2}$ \\
\hline 31 & $q(q+1)\left(q^{2}+q+1\right)\left(q^{4}+q^{3}+q^{2}+q+1\right)\left(q^{2}+1\right)$ \\
\hline 32 & $(q+1)\left(q^{2}+q+1\right)\left(q^{4}+q^{3}+q^{2}+q+1\right)\left(q^{2}+1\right)$ \\
\hline 33 & $q(q-1)\left(q^{2}+q+1\right)\left(q^{4}+q^{3}+q^{2}+q+1\right)\left(q^{2}+1\right)$ \\
\hline 34 & $(q-1)\left(q^{2}+q+1\right)\left(q^{4}+q^{3}+q^{2}+q+1\right)\left(q^{2}+1\right)$ \\
\hline 35 & $q(q+1)\left(q^{2}+1\right)\left(q^{4}+q^{3}+q^{2}+q+1\right)(q-1)^{2}$ \\
\hline 36 & $(q+1)\left(q^{2}+1\right)\left(q^{4}+q^{3}+q^{2}+q+1\right)(q-1)^{2}$ \\
\hline 37 & $\left(q^{2}+q+1\right)\left(q^{4}+q^{3}+q^{2}+q+1\right)\left(q^{2}+1\right)(q+1)^{2}$ \\
\hline 38 & $(q-1)(q+1)\left(q^{2}+q+1\right)\left(q^{4}+q^{3}+q^{2}+q+1\right)\left(q^{2}+1\right)$ \\
\hline 39 & $\left(q^{2}+q+1\right)\left(q^{4}+q^{3}+q^{2}+q+1\right)\left(q^{2}+1\right)(q-1)^{2}$ \\
\hline 40 & $\left(q^{2}+1\right)\left(q^{4}+q^{3}+q^{2}+q+1\right)(q-1)^{2}(q+1)^{2}$ \\
\hline 41 & $(q+1)\left(q^{2}+1\right)\left(q^{4}+q^{3}+q^{2}+q+1\right)(q-1)^{3}$ \\
\hline 42 & $(q+1)\left(q^{2}+q+1\right)\left(q^{4}+q^{3}+q^{2}+q+1\right)(q-1)^{3}$ \\
\hline 43 & $\left(q^{2}+1\right)\left(q^{2}+q+1\right)(q+1)^{2}(q-1)^{4}$ \\
\hline
\end{tabular}




\begin{tabular}{|c|c|c|}
\hline$h$ & $I_{h}$ & exceptions \\
\hline $1 \ldots, 7$ & $k_{1} \in \mathbb{Z}_{q-1}$ & \\
\hline $8, \ldots, 18$ & $\left(k_{1}, k_{2}\right) \in \mathbb{Z}_{q-1} \times \mathbb{Z}_{q-1}$ & $(q-1) \mid\left(k_{1}-k_{2}\right)$ \\
\hline $19,20,21$ & $\left(k_{1}, k_{2}, k_{3}\right) \in \mathbb{Z}_{q-1}^{3}$ & $(q-1) \mid\left(k_{i}-k_{j}\right), i \neq j$ \\
\hline $22,23,24$ & $\left(k_{1}, k_{2}\right) \in \mathbb{Z}_{q-1} \times \mathbb{Z}_{q^{2}-1}$ & $\begin{array}{l}\left(q^{2}-1\right) \mid(q+1) k_{1}-k_{2} \\
(q+1) \mid k_{2}\end{array}$ \\
\hline $25, \ldots, 28$ & $\left(k_{1}, k_{2}, k_{3}\right) \in \mathbb{Z}_{q-1}^{3}$ & $(q-1) \mid\left(k_{i}-k_{j}\right), i \neq j$ \\
\hline 29,30 & $\left(k_{1}, k_{2}\right) \in \mathbb{Z}_{q^{2}-1} \times \mathbb{Z}_{q-1}$ & $(q+1) \mid k_{1}$ \\
\hline 31,32 & $\left(k_{1}, k_{2}, k_{3}, k_{4}\right) \in \mathbb{Z}_{q-1}^{4}$ & $(q-1) \mid\left(k_{i}-k_{j}\right), i \neq j$ \\
\hline 33,34 & $\left(k_{1}, k_{2}, k_{3}\right) \in \mathbb{Z}_{q-1} \times \mathbb{Z}_{q^{2}-1} \times \mathbb{Z}_{q-1}$ & $\begin{array}{l}(q-1) \mid\left(k_{1}-k_{3}\right) \\
\left(q^{2}-1\right) \mid(q+1) k_{3}-k_{2} \\
\left(q^{2}-1\right) \mid(q+1) k_{1}-q k_{2} \\
(q+1) \mid k_{2}\end{array}$ \\
\hline 35,36 & $\left(k_{1}, k_{2}\right) \in \mathbb{Z}_{q-1} \times \mathbb{Z}_{q^{3}-1}$ & $\begin{array}{l}\left(q^{3}-1\right) \mid\left(q^{2}+q+1\right) k_{1}-k_{2} \\
\left(q^{2}+q+1\right) \mid(q+1) k_{2}\end{array}$ \\
\hline 37 & $\left(k_{1}, k_{2}, k_{3}, k_{4}, k_{5}\right) \in \mathbb{Z}_{q-1}^{5}$ & $(q-1) \mid k_{i}-k_{j}, i \neq j$ \\
\hline 38 & $\left(k_{1}, k_{2}, k_{3}, k_{4}\right) \in \mathbb{Z}_{q^{2}-1} \times \mathbb{Z}_{q-1}^{3}$ & $\begin{array}{l}(q+1) \mid k_{1} \\
(q-1) \mid k_{i}-k_{j}, 2 \leq i<j \leq 4\end{array}$ \\
\hline 39 & $\left(k_{1}, k_{2}, k_{3}\right) \in \mathbb{Z}_{q^{2}-1} \times \mathbb{Z}_{q-1} \times \mathbb{Z}_{q^{2}-1}$ & $\begin{array}{l}(q+1) \mid k_{1}, k_{3} \\
\left(q^{2}-1\right) \mid q k_{1}-k_{3} \\
\left(q^{2}-1\right) \mid k_{1}-k_{3}\end{array}$ \\
\hline 40 & $\left(k_{1}, k_{2}, k_{3}\right) \in \mathbb{Z}_{q^{3}-1} \times \mathbb{Z}_{q-1} \times \mathbb{Z}_{q-1}$ & $\begin{array}{l}\left(q^{2}+q+1\right) \mid(q+1) k_{1} \\
(q-1) \mid k_{2}-k_{3}\end{array}$ \\
\hline 41 & $\left(k_{1}, k_{2}\right) \in \mathbb{Z}_{q^{3}-1} \times \mathbb{Z}_{q^{2}-1}$ & $\begin{array}{l}\left(q^{2}+q+1\right) \mid(q+1) k_{1} \\
(q+1) \mid k_{2}\end{array}$ \\
\hline 42 & $\left(k_{1}, k_{2}\right) \in \mathbb{Z}_{q^{4}-1} \times \mathbb{Z}_{q-1}$ & $\begin{array}{l}\left(q^{3}+q^{2}+q+1\right) \mid\left(q^{2}+q+1\right) k_{1} \\
\left(q^{2}+1\right) \mid k_{1}\end{array}$ \\
\hline 43 & $k_{1} \in \mathbb{Z}_{q^{5}-1}$ & $\left(q^{5}-1\right) /(q-1) \mid\left(q^{4}-1\right) /(q-1) k_{1}$ \\
\hline
\end{tabular}

Table 11.13-B). Some $S y l_{p}$-decompositions of irreducible characters for $G L(5, q)$.

\begin{tabular}{c|c||c|c||c|c||c|c||c|c}
$h$ & $\mathbf{v}$ & $h$ & $\mathbf{v}$ & $h$ & $\mathbf{v}$ & $h$ & $\mathbf{v}$ & $h$ & $\mathbf{v}$ \\
\hline 26 & $(27)$ & 2 & $(6,23)$ & 8 & $(1,2)$ & 9 & $(3,14)$ & 10 & $(3,5)$ \\
11 & $(5,17)$ & 12 & $(6,7)$ & 13 & $(3,8)$ & 13 & $(14,22)$ & 13 & $(17,33)$ \\
14 & $(2,4)$ & 14 & $(17,23)$ & 14 & $(18,34)$ & 15 & $(5,9)$ & 15 & $(10,14)$ \\
15 & $(16,23)$ & 16 & $(3,11)$ & 16 & $(10,17)$ & 17 & $(4,6)$ & 17 & $(18,24)$ \\
18 & $(5,12)$ & 19 & $(8,9)$ & 19 & $(13,14)$ & 19 & $(21,38)$ & 20 & $(10,27)$ \\
20 & $(15,16)$ & 21 & $(11,12)$ & 21 & $(17,18)$ & 22 & $(24,39)$ & 25 & $(10,19)$ \\
25 & $(13,15)$ & 25 & $(27,33)$ & 25 & $(28,38)$ & 27 & $(9,11)$ & 27 & $(14,16)$ \\
27 & $(15,17)$ & 27 & $(28,34)$ & 28 & $(10,21)$ & 28 & $(16,18)$ & 29 & $(30,42)$ \\
31 & $(19,20)$ & 31 & $(25,27)$ & 31 & $(32,38)$ & 32 & $(20,21)$ & 32 & $(27,28)$ \\
33 & $(22,23)$ & 33 & $(34,39)$ & 34 & $(23,24)$ & 35 & $(36,41)$ & 37 & $(31,32)$ \\
38 & $(33,34)$ & 39 & $(29,30)$ & 40 & $(35,36)$ & 3 & $(5,24,30)$ & 4 & $(5,7,24)$ \\
1 & $(7,24,30,42)$ & & & & & & & &
\end{tabular}

Table (11.13-C). The $S y l_{p}$-vanishing characters of degree $\operatorname{St}(1)$ for $G L(5, q)$. 
Table (11.13-D). The $p$-vanishing characters of degree $S t(1)$ and their values for $G L(5, q)$ with $3 \mid(q+1)$.

In view of Lemma 9.2 we have the character $\psi=1_{G}+\chi_{24}(0, a)+\chi_{30}(a, 0)+$ $\chi_{42}\left(\left(q^{2}-1\right) b, 0\right)$. This character differs from $S t$ only on the following classes:

\begin{tabular}{l||l|l}
$C_{h}$ & $S t$ & $\psi$ \\
\hline$C_{22}\left(i_{1}, i_{2}\right)$ & $-q^{3}$ & $q^{3}-2 q^{3} \operatorname{Re}\left(\xi_{1}^{a i_{2}}\right)$ \\
$C_{29}\left(i_{1}, i_{2}\right)$ & $q^{2}$ & $3 q^{2}-2 q^{2} \operatorname{Re}\left(\xi^{a i_{1}}\right)$ \\
$C_{33}\left(i_{1}, i_{2}, i_{3}\right)$ & $-q$ & $q-2 q \operatorname{Re}\left(\xi_{1}^{a i_{2}}\right)$ \\
$C_{38}\left(i_{1}, i_{2}, i_{3}, i_{4}\right)$ & -1 & $1-2 \operatorname{Re}\left(\xi_{1}^{a i_{1}}\right)$ \\
$C_{39}\left(i_{1}, i_{2}, i_{3}\right)$ & 1 & $1-2\left(\operatorname{Re}\left(\xi_{1}^{a i_{1}}\right)+\operatorname{Re}\left(\xi_{1}^{a i_{3}}\right)-\operatorname{Re}\left(\xi_{1}^{\left(i_{1}+i_{3}\right) a}\right)-\operatorname{Re}\left(\xi_{1}^{\left(i_{1}-i_{3}\right) a}\right)\right)$ \\
$C_{41}\left(i_{1}, i_{2}\right)$ & -1 & $1-2 \operatorname{Re}\left(\xi_{1}^{a i_{2}}\right)$ \\
$C_{42}\left(i_{1}, i_{2}\right)$ & -1 & $1+2 \operatorname{Re}\left(\xi_{1}^{a i_{1}}\right)-2 \operatorname{Re}\left(\xi_{2}^{i_{1} b}\right)-2 \operatorname{Re}\left(\xi_{2}^{q i_{1} b}\right)$
\end{tabular}

11.14. Groups $G L(6, q)$.

Table (11.14 A). Some CHEviE sets $\mathbf{X}_{h}$, their degrees and parameters for $G L(6, q)$.

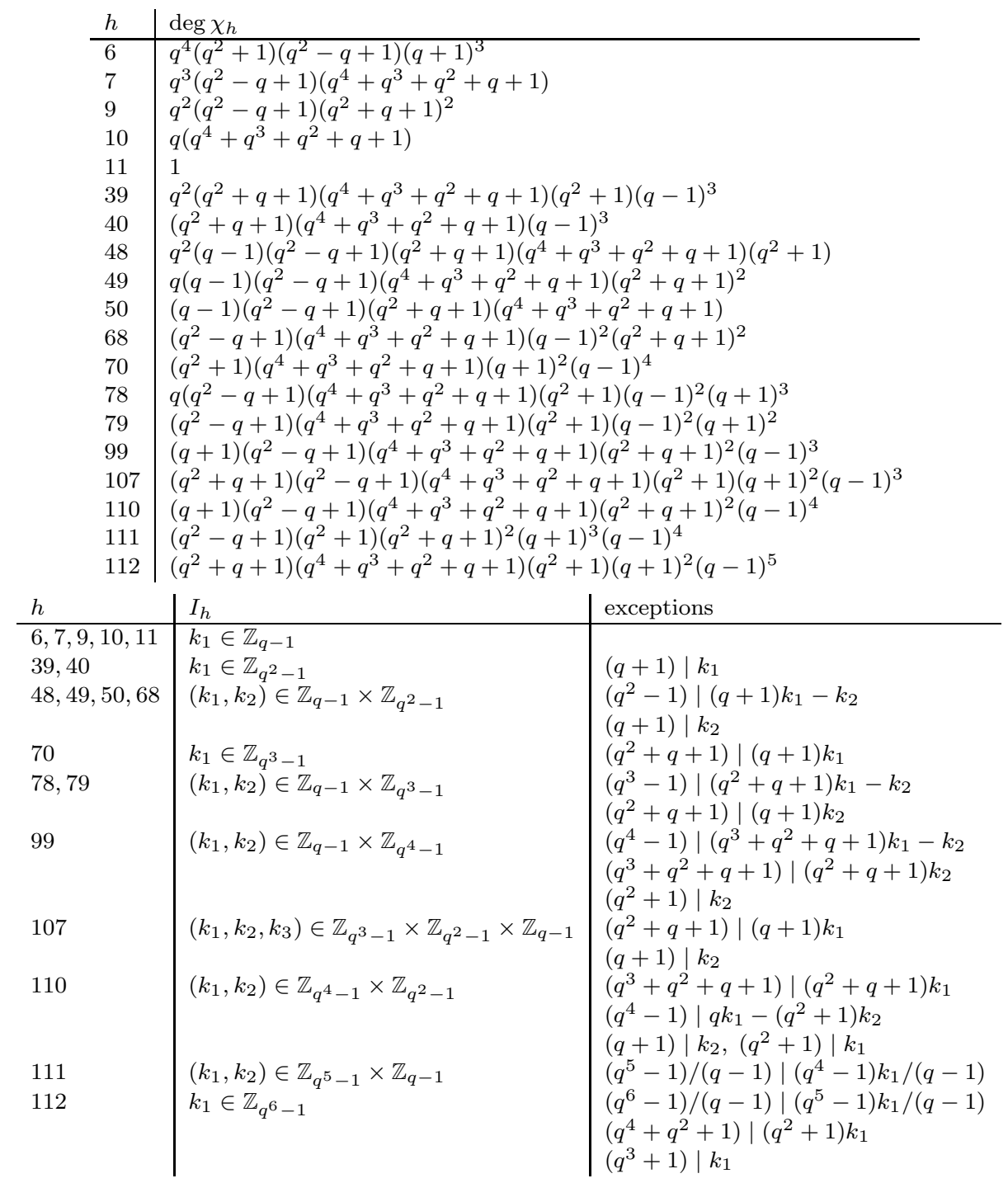


Table (11.14B). Some $S y l_{p}$-decompositions of irreducible characters for $G L(6, q)$.

\begin{tabular}{c|c||c|c||c|c||c|c||c|c}
$h$ & $\mathbf{v}$ & $h$ & $\mathbf{v}$ & $h$ & $\mathbf{v}$ & $h$ & $\mathbf{v}$ & $h$ & $\mathbf{v}$ \\
\hline 30 & $(32)$ & 31 & $(35)$ & 34 & $(36)$ & 58 & $(59)$ & 58 & $(61)$ \\
59 & $(61)$ & 60 & $(62)$ & 60 & $(63)$ & 62 & $(63)$ & 81 & $(82)$ \\
85 & $(86)$ & 12 & $(1,2)$ & 13 & $(3,20)$ & 13 & $(17,75)$ & 15 & $(6,35)$ \\
17 & $(9,27)$ & 18 & $(10,11)$ & 19 & $(3,12)$ & 19 & $(20,46)$ & 20 & $(2,5)$ \\
21 & $(4,32)$ & 21 & $(14,20)$ & 21 & $(22,47)$ & 22 & $(3,15)$ & 23 & $(24,48)$ \\
25 & $(9,15)$ & 25 & $(26,49)$ & 26 & $(7,36)$ & 26 & $(16,27)$ & 27 & $(8,10)$ \\
27 & $(28,50)$ & 28 & $(9,18)$ & 29 & $(4,19)$ & 29 & $(35,74)$ & 32 & $(6,13)$ \\
32 & $(36,75)$ & 33 & $(35,43)$ & 35 & $(5,8)$ & 35 & $(37,76)$ & 36 & $(6,17)$ \\
37 & $(7,28)$ & 38 & $(40,110)$ & 41 & $(12,13)$ & 41 & $(19,20)$ & 42 & $(14,52)$ \\
42 & $(21,22)$ & 43 & $(14,16)$ & 43 & $(23,24)$ & 44 & $(16,55)$ & 44 & $(25,26)$ \\
45 & $(17,18)$ & 45 & $(27,28)$ & 51 & $(14,41)$ & 51 & $(19,21)$ & 51 & $(29,32)$ \\
51 & $(52,74)$ & 51 & $(55,94)$ & 52 & $(13,15)$ & 52 & $(20,22)$ & 52 & $(32,35)$ \\
52 & $(55,75)$ & 52 & $(56,95)$ & 53 & $(16,42)$ & 53 & $(23,61)$ & 53 & $(32,33)$ \\
53 & $(43,52)$ & 53 & $(54,75)$ & 54 & $(14,44)$ & 54 & $(24,63)$ & 54 & $(33,36)$ \\
54 & $(43,55)$ & 55 & $(15,17)$ & 55 & $(25,27)$ & 55 & $(35,36)$ & 55 & $(56,76)$ \\
56 & $(16,45)$ & 56 & $(26,28)$ & 56 & $(36,37)$ & 57 & $(23,51)$ & 57 & $(61,84)$ \\
57 & $(63,94)$ & 61 & $(21,25)$ & 61 & $(24,52)$ & 61 & $(63,86)$ & 61 & $(64,95)$ \\
63 & $(22,26)$ & 63 & $(23,55)$ & 63 & $(64,87)$ & 64 & $(24,56)$ & 65 & $(66,98)$ \\
65 & $(67,90)$ & 66 & $(68,91)$ & 67 & $(68,99)$ & 69 & $(70,112)$ & 71 & $(41,42)$ \\
71 & $(51,52)$ & 71 & $(73,103)$ & 72 & $(43,82)$ & 72 & $(53,54)$ & 73 & $(44,45)$ \\
73 & $(55,56)$ & 74 & $(46,47)$ & 74 & $(76,104)$ & 75 & $(48,86)$ & 76 & $(49,50)$ \\
77 & $(79,107)$ & 80 & $(43,71)$ & 80 & $(51,53)$ & 80 & $(57,61)$ & 80 & $(82,94)$ \\
80 & $(83,103)$ & 82 & $(42,44)$ & 82 & $(52,54)$ & 82 & $(53,55)$ & 82 & $(61,63)$ \\
82 & $(83,95)$ & 83 & $(43,73)$ & 83 & $(54,56)$ & 83 & $(63,64)$ & 84 & $(48,74)$ \\
84 & $(86,100)$ & 84 & $(87,104)$ & 86 & $(47,49)$ & 86 & $(87,101)$ & 87 & $(48,76)$ \\
88 & $(65,67)$ & 88 & $(89,109)$ & 89 & $(66,68)$ & 90 & $(38,39)$ & 90 & $(91,110)$ \\
91 & $(39,40)$ & 92 & $(71,72)$ & 92 & $(80,82)$ & 92 & $(93,103)$ & 93 & $(72,73)$ \\
93 & $(82,83)$ & 94 & $(74,75)$ & 94 & $(84,86)$ & 94 & $(95,104)$ & 95 & $(75,76)$ \\
95 & $(86,87)$ & 96 & $(77,78)$ & 96 & $(97,107)$ & 97 & $(78,79)$ & 98 & $(99,110)$ \\
100 & $(65,66)$ & 100 & $(101,105)$ & 101 & $(67,68)$ & 102 & $(92,93)$ & 103 & $(94,95)$ \\
104 & $(88,89)$ & 104 & $(100,101)$ & 105 & $(90,91)$ & 106 & $(96,97)$ & 108 & $(69,70)$ \\
109 & $(98,99)$ & 4 & $(7,50,68)$ & 5 & $(7,10,49)$ & 8 & $(9,11,50)$ & 14 & $(3,4,6)$
\end{tabular}

\begin{tabular}{c|c||c|c||c|c}
$h$ & $\mathbf{v}$ & $h$ & $\mathbf{v}$ & $h$ & $\mathbf{v}$ \\
\hline 16 & $(6,7,9)$ & 24 & $(4,6,9)$ & 24 & $(16,50,68)$ \\
3 & $(9,48,50,68)$ & 2 & $(10,39,49,68,99)$ & 47 & $(48,50,68,68,99)$ \\
1 & $(11,40,50,68,99,110)$ & 46 & $(39,40,50,68,68,99,110)$ & &
\end{tabular}

Table $(11.14 \mathrm{C})$. The $S y l_{p}$-vanishing characters of degree $S t(1)$ for $G L(6, q)$.

\begin{tabular}{c|c||c|c||c|c}
$j$ & $\mathbf{v}$ & $j$ & $\mathbf{v}$ & $j$ & $\mathbf{v}$ \\
\hline 1 & $(11,40,50,68,99,110)$ & 2 & $(11,40,50,67,110)$ & 3 & $(11,38,50,68,99)$ \\
4 & $(11,40,50,68,98)$ & 5 & $(11,38,50,67)$ & 6 & $(1)$
\end{tabular}

\subsection{Groups $C S p(6, q)$.}

Table (11.15. A). Some CHEviE sets $\mathbf{X}_{h}$, their degrees and parameters for $C \operatorname{Sp}(6, q)$ with $q$ odd.

\begin{tabular}{l|l||l|l}
$h$ & $\operatorname{deg} \chi_{h}$ & $k$ & $\operatorname{deg} \chi_{h}$ \\
\hline 1 & 1 & 4 & $\frac{1}{2} q\left(q^{2}-q+1\right)(q+1)^{2}$ \\
5 & $\frac{1}{2}(q-1)^{2} q\left(q^{2}+q+1\right)$ & 7 & $q^{3}\left(q^{2}-q+1\right)\left(q^{2}+q+1\right)$ \\
10 & $\frac{1}{2} q^{4}\left(q^{2}-q+1\right)\left(q^{2}+1\right)$ & 11 & $\frac{1}{2}(q-1)^{2} q^{4}\left(q^{2}+q+1\right)$ \\
18 & $(q-1)\left(q^{2}+q+1\right)$ & 45 & $\frac{1}{2}(q-1)^{3} q\left(q^{2}+q+1\right)\left(q^{2}-q+1\right)$ \\
50 & $(q-1)^{2}\left(q^{2}+q+1\right)\left(q^{2}+1\right)$ & 51 & $(q-1)^{3} q\left(q^{2}+1\right)\left(q^{2}+q+1\right)$ \\
65 & $(q-1)\left(q^{2}-q+1\right)\left(q^{2}+1\right)\left(q^{2}+q+1\right)$ & 71 & $(q-1)^{2}(q+1)\left(q^{2}+q+1\right)\left(q^{2}-q+1\right)$ \\
97 & $(q-1)^{2}(q+1)^{3}\left(q^{2}+1\right)\left(q^{2}-q+1\right)$ & 98 & $(q-1)^{3}\left(q^{2}+q+1\right)\left(q^{2}+1\right)\left(q^{2}-q+1\right)$
\end{tabular}




\begin{tabular}{l|l|l}
$h$ & $I_{h}$ & exceptions \\
\hline $1,4,5,7,10,11,18$ & $k_{1} \in \mathbb{Z}_{q-1}$ & \\
45 & $\left(k_{1}, k_{2}\right) \in \mathbb{Z}_{q-1} \times \mathbb{Z}_{q-1}$ & $(q+1) \mid k_{1}$ \\
$50,51,65,71$ & $\left(k_{1}, k_{2}\right) \in \mathbb{Z}_{q+1} \times \mathbb{Z}_{q-1}$ & $\frac{q+1}{2} \mid k_{1}$ \\
97 & $\left(k_{1}, k_{2}\right) \in \mathbb{Z}_{q^{3}-1} \times \mathbb{Z}_{q-1}$ & $\left(q^{2}+q+1\right) \mid k_{1}$ \\
98 & $\left(k_{1}, k_{2}, k_{3}, k_{4}\right) \in \mathbb{Z}_{q+1}^{3} \times \mathbb{Z}_{q-1}$ & $(q+1) \mid k_{1}, k_{2}, k_{3}$ \\
& & $(q+1) \mid\left(k_{i} \pm k_{j}\right), 1 \leq i<j \leq 3$
\end{tabular}

Table (11.15-B). Some $S y l_{p}$-decompositions of irreducible characters for $C S p(6, q)$, $q$ odd.

\begin{tabular}{c|c||c|c||c|c||c|c||c|c||c|c}
$h$ & $\mathbf{v}$ & $h$ & $\mathbf{v}$ & $h$ & $\mathbf{v}$ & $h$ & $\mathbf{v}$ & $h$ & $\mathbf{v}$ & $h$ & $\mathbf{v}$ \\
\hline 24 & $(26)$ & 27 & $(29)$ & 54 & $(55)$ & 62 & $(63)$ & 9 & $(4,42)$ & 14 & $(23,41)$ \\
14 & $(31,35)$ & 16 & $(37,42)$ & 19 & $(18,50)$ & 20 & $(50,61)$ & 21 & $(39,44)$ & 22 & $(21,52)$ \\
23 & $(19,35)$ & 25 & $(23,61)$ & 26 & $(23,65)$ & 28 & $(23,79)$ & 28 & $(26,66)$ & 28 & $(37,44)$ \\
29 & $(25,67)$ & 29 & $(26,63)$ & 30 & $(16,46)$ & 30 & $(25,80)$ & 30 & $(28,64)$ & 30 & $(29,68)$ \\
31 & $(19,41)$ & 32 & $(31,71)$ & 33 & $(31,89)$ & 33 & $(39,42)$ & 34 & $(21,46)$ & 34 & $(32,90)$ \\
34 & $(33,72)$ & 38 & $(14,42)$ & 38 & $(25,43)$ & 39 & $(5,11)$ & 40 & $(16,34)$ & 40 & $(21,30)$ \\
42 & $(32,39)$ & 43 & $(19,39)$ & 47 & $(13,14)$ & 48 & $(15,55)$ & 49 & $(16,17)$ & 49 & $(47,93)$ \\
52 & $(51,86)$ & 53 & $(15,47)$ & 53 & $(23,25)$ & 55 & $(14,16)$ & 55 & $(26,29)$ & 55 & $(36,39)$ \\
55 & $(37,38)$ & 55 & $(53,83)$ & 56 & $(15,49)$ & 56 & $(28,30)$ & 56 & $(53,93)$ & 56 & $(55,84)$ \\
57 & $(23,26)$ & 58 & $(25,29)$ & 58 & $(57,77)$ & 59 & $(26,28)$ & 59 & $(57,79)$ & 60 & $(29,30)$ \\
63 & $(61,85)$ & 64 & $(20,52)$ & 64 & $(61,95)$ & 64 & $(63,86)$ & 66 & $(65,87)$ & 67 & $(65,85)$ \\
68 & $(66,86)$ & 68 & $(67,88)$ & 69 & $(31,32)$ & 70 & $(33,34)$ & 70 & $(69,96)$ & 72 & $(71,99)$ \\
73 & $(47,48)$ & 73 & $(53,55)$ & 73 & $(57,58)$ & 74 & $(48,49)$ & 74 & $(55,56)$ & 74 & $(59,60)$ \\
74 & $(73,93)$ & 75 & $(57,59)$ & 76 & $(58,60)$ & 76 & $(75,92)$ & 77 & $(61,63)$ & 78 & $(63,64)$ \\
78 & $(77,95)$ & 79 & $(65,66)$ & 80 & $(67,68)$ & 80 & $(79,95)$ & 81 & $(31,33)$ & 82 & $(32,34)$ \\
82 & $(81,94)$ & 83 & $(65,67)$ & 84 & $(66,68)$ & 84 & $(83,94)$ & 85 & $(50,51)$ & 86 & $(85,98)$ \\
88 & $(87,98)$ & 90 & $(89,99)$ & 91 & $(73,74)$ & 91 & $(75,76)$ & 92 & $(69,70)$ & 92 & $(77,78)$ \\
92 & $(81,82)$ & 93 & $(79,80)$ & 93 & $(83,84)$ & 94 & $(71,72)$ & 94 & $(87,88)$ & 95 & $(50,52)$ \\
95 & $(85,86)$ & 96 & $(89,90)$ & & & & & & & &
\end{tabular}

\begin{tabular}{c|c||c|c||c|c||c|c}
$h$ & $\mathbf{v}$ & $h$ & $\mathbf{v}$ & $h$ & $\mathbf{v}$ & $h$ & $\mathbf{v}$ \\
\hline 2 & $(1,4,18)$ & 3 & $(1,5,18)$ & 8 & $(5,7,44)$ & 12 & $(10,11,46)$ \\
13 & $(1,1,18)$ & 17 & $(10,10,46)$ & 35 & $(1,2,4)$ & 35 & $(4,4,13)$ \\
37 & $(4,7,10)$ & 41 & $(5,5,18)$ & 44 & $(41,45,71)$ & 61 & $(41,41,71)$ \\
87 & $(45,45,71)$ & 100 & $(51,51,51,98)$ & 89 & $(5,5,11,11,71)$ & 99 & $(45,45,45,45,98)$
\end{tabular}

\begin{tabular}{c|c||c|c}
$h$ & $\mathbf{v}$ & $h$ & $\mathbf{v}$ \\
\hline 6 & $(1,4,5,18,18,50)$ & 15 & $(4,4,5,5,18,18,50,71)$ \\
46 & $(5,5,18,45,45,50,51,71,98)$ & 46 & $(5,11,18,45,45,45,50,71,98)$
\end{tabular}

Table (11.15 C). The $S y l_{p}$-vanishing characters of degree $\operatorname{St}(1)$ for $C S p(6, q)$ with $q$ odd.

\begin{tabular}{c|c||c|c}
$j$ & $\mathbf{v}$ & $j$ & $\mathbf{v}$ \\
\hline 1 & $(1,5,5,18,45,45,50,51,51,65,71,98)$ & 2 & $(1,5,5,18,45,45,51,65,71,85,98)$ \\
3 & $(1,5,5,18,45,45,51,65,71,86)$ & 4 & $(1,5,5,18,45,45,51,67,71,98)$ \\
5 & $(1,5,5,18,45,45,52,65,71)$ & 6 & $(1,5,5,18,50,51,51,65,87,98)$ \\
7 & $(1,5,5,18,50,51,51,65,88)$ & 8 & $(1,5,5,18,50,51,51,66,98)$ \\
9 & $(1,5,5,18,51,65,85,87,98)$ & 10 & $(1,5,5,18,51,65,85,88)$ \\
11 & $(1,5,5,18,51,65,86,87)$ & 12 & $(1,5,5,18,51,66,85,98)$ \\
13 & $(1,5,5,18,51,66,86)$ & 14 & $(1,5,5,18,51,67,87,98)$ \\
15 & $(1,5,5,18,51,67,88)$ & 16 & $(1,5,5,18,51,68)$ \\
17 & $(1,5,5,18,52,65,87)$ & 18 & $(1,5,5,18,52,66)$ \\
19 & $(1,5,5,19,45,45,51,51,65,71,98)$ & 20 & $(1,5,5,19,51,51,65,87,98)$ \\
21 & $(1,5,5,19,51,51,65,88)$ & 22 & $(1,5,5,19,51,51,66,98)$ \\
23 & $(1,5,11,18,45,45,45,50,51,65,71,98)$ & 24 & $(1,5,11,18,45,45,45,65,71,85,98)$
\end{tabular}




\begin{tabular}{|c|c|c|c|}
\hline$j$ & $\mathbf{v}$ & $j$ & $\mathbf{v}$ \\
\hline 25 & $(1,5,11,18,45,45,45,65,71,86)$ & 26 & $(1,5,11,18,45,45,45,67,71,98)$ \\
\hline 27 & $(1,5,11,18,45,50,51,65,87,98)$ & 28 & $(1,5,11,18,45,50,51,65,88)$ \\
\hline 29 & $(1,5,11,18,45,50,51,66,98)$ & 30 & $(1,5,11,18,45,65,85,87,98)$ \\
\hline 31 & $(1,5,11,18,45,65,85,88)$ & 32 & $(1,5,11,18,45,65,86,87)$ \\
\hline 33 & $(1,5,11,18,45,66,85,98)$ & 34 & $(1,5,11,18,45,66,86)$ \\
\hline 35 & $(1,5,11,18,45,67,87,98)$ & 36 & $(1,5,11,18,45,67,88)$ \\
\hline 37 & $(1,5,11,18,45,68)$ & 38 & $(1,5,11,19,45,45,45,51,65,71,98)$ \\
\hline 39 & $(1,5,11,19,45,51,65,87,98)$ & 40 & $(1,5,11,19,45,51,65,88)$ \\
\hline 41 & $(1,5,11,19,45,51,66,98)$ & 42 & $(1,11,11,18,45,45,45,45,50,65,71,98)$ \\
\hline 43 & $(1,11,11,18,45,45,50,65,87,98)$ & 44 & $(1,11,11,18,45,45,50,65,88)$ \\
\hline 45 & $(1,11,11,18,45,45,50,66,98)$ & 46 & $(1,11,11,18,50,65,71,99)$ \\
\hline 47 & $(1,11,11,18,50,65,72)$ & 48 & $(1,11,11,19,45,45,45,45,65,71,98)$ \\
\hline 49 & $(1,11,11,19,45,45,65,87,98)$ & 50 & $(1,11,11,19,45,45,65,88)$ \\
\hline 51 & $(1,11,11,19,45,45,66,98)$ & 52 & $(1,11,11,19,65,71,99)$ \\
\hline 53 & $(1,11,11,19,65,72)$ & 54 & $(1,18,39,45,45,45,50,51,65,71,98)$ \\
\hline 55 & $(1,18,39,45,45,45,65,71,85,98)$ & 56 & $(1,18,39,45,45,45,65,71,86)$ \\
\hline 57 & $(1,18,39,45,45,45,67,71,98)$ & 58 & $(1,18,39,45,50,51,65,87,98)$ \\
\hline 59 & $(1,18,39,45,50,51,65,88)$ & 60 & $(1,18,39,45,50,51,66,98)$ \\
\hline 61 & $(1,18,39,45,65,85,87,98)$ & 62 & $(1,18,39,45,65,85,88)$ \\
\hline 63 & $(1,18,39,45,65,86,87)$ & 64 & $(1,18,39,45,66,85,98)$ \\
\hline 65 & $(1,18,39,45,66,86)$ & 66 & $(1,18,39,45,67,87,98)$ \\
\hline 67 & $(1,18,39,45,67,88)$ & 68 & $(1,18,39,45,68)$ \\
\hline 69 & $(1,19,39,45,45,45,51,65,71,98)$ & 70 & $(1,19,39,45,51,65,87,98)$ \\
\hline 71 & $(1,19,39,45,51,65,88)$ & 72 & $(1,19,39,45,51,66,98)$ \\
\hline 73 & $(1,41,45,45,50,51,51,65,71,98)$ & 74 & $(1,41,45,45,51,65,71,85,98)$ \\
\hline 75 & $(1,41,45,45,51,65,71,86)$ & 76 & $(1,41,45,45,51,67,71,98)$ \\
\hline 77 & $(1,41,45,45,52,65,71)$ & 78 & $(1,41,50,51,51,65,87,98)$ \\
\hline 79 & $(1,41,50,51,51,65,88)$ & 80 & $(1,41,50,51,51,66,98)$ \\
\hline 81 & $(1,41,51,65,85,87,98)$ & 82 & $(1,41,51,65,85,88)$ \\
\hline 83 & $(1,41,51,65,86,87)$ & 84 & $(1,41,51,66,85,98)$ \\
\hline 85 & $(1,41,51,66,86)$ & 86 & $(1,41,51,67,87,98)$ \\
\hline 87 & $(1,41,51,67,88)$ & 88 & $(1,41,51,68)$ \\
\hline 89 & $(1,41,52,65,87)$ & 90 & $(1,41,52,66)$ \\
\hline 91 & $(1,43,45,45,45,51,65,71,98)$ & 92 & $(1,43,45,51,65,87,98)$ \\
\hline 93 & $(1,43,45,51,65,88)$ & 94 & $(1,43,45,51,66,98)$ \\
\hline 95 & $(1,44,45,50,51,51,65,98)$ & 96 & $(1,44,45,51,65,85,98)$ \\
\hline 97 & $(1,44,45,51,65,86)$ & 98 & $(1,44,45,51,67,98)$ \\
\hline 99 & $(1,44,45,52,65)$ & 100 & $(1,46,51,65)$ \\
\hline 101 & $(3,5,45,45,50,51,51,65,71,98)$ & 102 & $(3,5,45,45,51,65,71,85,98)$ \\
\hline 103 & $(3,5,45,45,51,65,71,86)$ & 104 & $(3,5,45,45,51,67,71,98)$ \\
\hline 105 & $(3,5,45,45,52,65,71)$ & 106 & $(3,5,50,51,51,65,87,98)$ \\
\hline 107 & $(3,5,50,51,51,65,88)$ & 108 & $(3,5,50,51,51,66,98)$ \\
\hline 109 & $(3,5,51,65,85,87,98)$ & 110 & $(3,5,51,65,85,88)$ \\
\hline 111 & $(3,5,51,65,86,87)$ & 112 & $(3,5,51,66,85,98)$ \\
\hline 113 & $(3,5,51,66,86)$ & 114 & $(3,5,51,67,87,98)$ \\
\hline 115 & $(3,5,51,67,88)$ & 116 & $(3,5,51,68)$ \\
\hline 117 & $(3,5,52,65,87)$ & 118 & $(3,5,52,66)$ \\
\hline 119 & $(3,11,45,45,45,50,51,65,71,98)$ & 120 & $(3,11,45,45,45,65,71,85,98)$ \\
\hline 121 & $(3,11,45,45,45,65,71,86)$ & 122 & $(3,11,45,45,45,67,71,98)$ \\
\hline 123 & $(3,11,45,50,51,65,87,98)$ & 124 & $(3,11,45,50,51,65,88)$ \\
\hline 125 & $(3,11,45,50,51,66,98)$ & 126 & $(3,11,45,65,85,87,98)$ \\
\hline 127 & $(3,11,45,65,85,88)$ & 128 & $(3,11,45,65,86,87)$ \\
\hline 129 & $(3,11,45,66,85,98)$ & 130 & $(3,11,45,66,86)$ \\
\hline 131 & $(3,11,45,67,87,98)$ & 132 & $(3,11,45,67,88)$ \\
\hline 133 & $(3,11,45,68)$ & 134 & $(5,5,5,10,18,45,50,51,51,71,98)$ \\
\hline 135 & $(5,5,5,10,18,45,51,71,85,98)$ & 136 & $(5,5,5,10,18,45,51,71,86)$ \\
\hline 137 & $(5,5,5,10,18,45,52,71)$ & 138 & $(5,5,5,10,19,45,51,51,71,98)$ \\
\hline 139 & $(5,5,10,11,18,45,45,50,51,71,98)$ & 140 & $(5,5,10,11,18,45,45,71,85,98)$ \\
\hline 141 & $(5,5,10,11,18,45,45,71,86)$ & 142 & $(5,5,10,11,18,50,51,87,98)$ \\
\hline
\end{tabular}




\begin{tabular}{c|c||c|c}
$j$ & $\mathbf{v}$ & $j$ & $\mathbf{v}$ \\
\hline 143 & $(5,5,10,11,18,50,51,88)$ & 144 & $(5,5,10,11,18,85,87,98)$ \\
145 & $(5,5,10,11,18,85,88)$ & 146 & $(5,5,10,11,18,86,87)$ \\
147 & $(5,5,10,11,19,45,45,51,71,98)$ & 148 & $(5,5,10,11,19,51,87,98)$ \\
149 & $(5,5,10,11,19,51,88)$ & 150 & $(5,10,11,11,18,45,45,45,50,71,98)$ \\
151 & $(5,10,11,11,18,45,50,87,98)$ & 152 & $(5,10,11,11,18,45,50,88)$ \\
153 & $(5,10,11,11,19,45,45,45,71,98)$ & 154 & $(5,10,11,11,19,45,87,98)$ \\
155 & $(5,10,11,11,19,45,88)$ & 156 & $(5,10,18,39,45,45,50,51,71,98)$ \\
157 & $(5,10,18,39,45,45,71,85,98)$ & 158 & $(5,10,18,39,45,45,71,86)$ \\
159 & $(5,10,18,39,50,51,87,98)$ & 160 & $(5,10,18,39,50,51,88)$ \\
161 & $(5,10,18,39,85,87,98)$ & 162 & $(5,10,18,39,85,88)$ \\
163 & $(5,10,18,39,86,87)$ & 164 & $(5,10,19,39,45,45,51,71,98)$ \\
165 & $(5,10,19,39,51,87,98)$ & 166 & $(5,10,19,39,51,88)$ \\
167 & $(5,10,41,45,50,51,51,71,98)$ & 168 & $(5,10,41,45,51,71,85,98)$ \\
169 & $(5,10,41,45,51,71,86)$ & 170 & $(5,10,41,45,52,71)$ \\
171 & $(5,10,43,45,45,51,71,98)$ & 172 & $(5,10,43,51,87,98)$ \\
173 & $(5,10,43,51,88)$ & 174 & $(5,10,44,50,51,51,98)$ \\
175 & $(5,10,44,51,85,98)$ & 176 & $(5,10,44,51,86)$ \\
177 & $(5,10,44,52)$ & 178 & $(10,11,18,39,45,45,45,50,71,98)$ \\
179 & $(10,11,18,39,45,50,87,98)$ & 180 & $(10,11,18,39,45,50,88)$ \\
181 & $(10,11,19,39,45,45,45,71,98)$ & 182 & $(10,11,19,39,45,87,98)$ \\
183 & $(10,11,19,39,45,88)$ & 184 & $(10,11,41,45,45,50,51,71,98)$ \\
185 & $(10,11,41,45,45,71,85,98)$ & 186 & $(10,11,41,45,45,71,86)$ \\
187 & $(10,11,41,50,51,87,98)$ & 188 & $(10,11,41,50,51,88)$ \\
189 & $(10,11,41,85,87,98)$ & 190 & $(10,11,41,85,88)$ \\
191 & $(10,11,41,86,87)$ & 192 & $(10,11,43,45,45,45,71,98)$ \\
193 & $(10,11,43,45,87,98)$ & 194 & $(10,11,43,45,88)$ \\
195 & $(10,11,44,45,50,51,98)$ & 196 & $(10,11,44,45,85,98)$ \\
197 & $(10,11,44,45,86)$ & 198 & $(10,11,46)$ \\
199 & & & \\
& & &
\end{tabular}

\section{REFERENCES}

[1] F. M. Bleher, Finite groups of Lie type of small rank, Pacific J. Math. 187 (1999), no. 2, 215-239.

[2] R.W. Carter, Finite Groups of Lie type. Conjugacy Classes and Complex Characters, John Wiley \& Sons, Chichester, 1985.

[3] J.H. Conway, R.T. Curtis, S.P. Norton, R.A. Parker and R.A. Wilson, Atlas of finite groups. Maximal subgroups and ordinary characters for simple groups, Oxford University Press, Eynsham, 1985.

[4] Ch.W. Curtis, N. Iwahori and R. Kilmoyer, Hecke algebras and characters of parabolic type of finite groups with (B,N)-pairs, Publ. Math. IHES 40 (1972), 81-116.

[5] Ch.W. Curtis and I. Reiner, Methods of representation theory. With applications to finite groups and orders, Vol. 1, John Wiley \& Sons, New York, 1981.

[6] Ch.W. Curtis and I. Reiner, Methods of representation theory. With applications to finite groups and orders, Vol. 2, John Wiley \& Sons, New York, 1987.

[7] F. Digne and J. Michel, Representations of finite groups of Lie type, London Math. Soc. Student Texts no. 21, Cambridge University Press, 1991.

[8] W. Feit, The representation theory of finite groups, North-Holland Mathematical Library, Amsterdam, 1982.

[9] M. Geck, G. Hiss, F. Lübeck, G. Malle, and G. Pfeiffer, CHevie - A system for computing and processing generic character tables for finite groups of Lie type, Weyl groups and Hecke algebras, Appl. Algebra Engrg. Comm. Comput., 7 (1996), 175-210.

[10] I.M. Gelfand and M.I. Graev, Construction of irreducible representations of simple algebraic groups over finite fields, Dokl. Acad. Nauk SSSR 147 (1962), 529-532 (Soviet Math. Doklady 3(1962), 1646-1648).

[11] D. Gorenstein, R. Lyons and R. Solomon, The Classification of Finite Simple Groups, No. 3, Part I, Chapter A, Amer. Math. Soc. Providence, R.I. 1998. 
[12] F. Himstedt and S.-C. Huang, Dade's invariant conjecture for the Ree groups ${ }^{2} F_{4}(q)$ in defining characteristic, Comm. Algebra, 40 (2012), 452-496.

[13] J. Humphreys, Modular representations of finite groups of Lie type, Cambridge Univ. Press, Cambridge, 2006.

[14] D. Kotlar, On the irreducible constituents of degenerate Gelfand-Graev characters, J. Algebra 173 (1995), 348-360.

[15] D. Lusztig, On the representations of reductive groups with disconnected centre. Orbites unipotentes et représentations, I, Astérisque No. 168 (1988), 10, 157-166.

[16] G. Malle and D. Testerman, Linear algebraic groups and finite groups of Lie type, Cambridge Univ. Press, Cambridge, 2011.

[17] G. Malle and Th. Weigel, Finite groups with minimal 1-PIM, Manuscripta Math, 126 (2008), 315-332.

[18] G. Malle and A.E. Zalesskiı̌, Prime power degree representations of quasi-simple groups, Arch. Math. (Basel) 77 (2001), 461-468.

[19] A. Przygocki, Schur indices of symplectic groups, Comm. Algebra 10 (1982), no. 3, $279-310$

[20] K. Shinoda, The characters of the finite conformal symplectic group CSp(4,q), Comm. Algebra 10 (1982), no. 13, 1369-1419.

[21] B. Srinivasan, The characters of the finite symplectic group $S p(4, q)$, Trans. Amer. Math. Soc. 131 (1968) 488-525.

[22] R. Steinberg, Lectures on Chevalley groups, Yale University, New Haven, 1968.

[23] F.D. Veldkamp, Regular elements in anisotropic tori, in Contributions to algebra (collection of papers dedicated to Ellis Kolchin), Academic Press, New York, 1977, 389-424.

[24] A. Zalesski, Low dimensional projective indecomposable modules for Chevalley groups in defining characteristic, J. Algebra 377 (2013), 125-156.

Departamento de Matemática, Universidade de Brasília, 70910-900 Brasília - DF, BRAZIL

E-mail address: pellegrini@unb.br

Università degli Studi di Milano-Bicocca, Dipartimento di Matematica e Applicazioni, via R.CozZi 53, 20125 Milano, Italy

E-mail address: alexandre.zalesski@gmail.com 